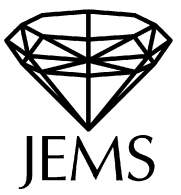

Octav Cornea $\cdot$ Andrew Ranicki

\title{
Rigidity and gluing for Morse and Novikov complexes
}

Received September 19, 2002 / final version received May 12, 2003

Published online July 31, 2003 - (c) Springer-Verlag \& EMS 2003

\begin{abstract}
We obtain rigidity and gluing results for the Morse complex of a real-valued Morse function as well as for the Novikov complex of a circle-valued Morse function. A rigidity result is also proved for the Floer complex of a hamiltonian defined on a closed symplectic manifold $(M, \omega)$ with $\left.c_{1}\right|_{\pi_{2}(M)}=\left.[\omega]\right|_{\pi_{2}(M)}=0$. The rigidity results for these complexes show that the complex of a fixed generic function/hamiltonian is a retract of the Morse (respectively Novikov or Floer) complex of any other sufficiently $C^{0}$ close generic function/hamiltonian. The gluing result is a type of Mayer-Vietoris formula for the Morse complex. It is used to express algebraically the Novikov complex up to isomorphism in terms of the Morse complex of a fundamental domain. Morse cobordisms are used to compare various Morse-type complexes without the need of bifurcation theory.
\end{abstract}

\section{Contents}

Introduction . . . . . . . . . . . . . . . . . . . . . . . 343

1 Cobordism of Morse functions . . . . . . . . . . . . . . . . . . 345

1.1 Basic constructions . . . . . . . . . . . . . . . . . 345

1.2 Rigidity of the Morse complex . . . . . . . . . . . . . . . . . . 357

1.3 Mayer-Vietoris type formula for the Morse complex . . . . . . . . . . . 361

2 Applications . . . . . . . . . . . . . . . . . . . . . . . . . 374

2.1 Rigidity of the Floer complex . . . . . . . . . . . . . . . . . . . . . . . . . . . . . . . . . . . . . . . . .

2.2 Circle-valued functions . . . . . . . . . . . . . . . . . . . . . . . . 379

References . . . . . . . . . . . . . . . . . . . . . . . . . . . . 394

\section{Introduction}

A Morse type complex associated to a generic pair $(f, \alpha)$ with $f$ a functional and with $\alpha$ an additional geometric structure is a complex freely generated by the critical points (assumed in the paper to be finite in number) of $f$ and with a differential given by counting "flow lines" that join successive critical points of $f$. The definition of these "flow lines" depends on both $f$ and $\alpha$. For example, $f$ might be a Morse

O. Cornea: University of Montréal, Department of Mathematics and Statistics, CP 6128 Succ. Centre Ville, Montréal, QC H3C 3J7, Canada, e-mail: cornea@dms.umontreal.ca

A. Ranicki: School of Mathematics, University of Edinburgh, King's Buildings, Mayfield Road, Edinburgh EH9 3JZ, Scotland, UK, e-mail: aar@maths.ed.ac.uk 
function, $\alpha$ a Riemannian metric and then the differential counts flow lines of $-\nabla^{\alpha} f$. The complex is in this case the classical Morse complex variously attributed to Thom, Smale, Milnor and under a different form to Witten. Other examples that will appear in this paper are provided by the Novikov complex where $f$ is an $S^{1}$-valued function (the additional structure being again a metric) and the Floer complex which, from this perspective, is associated to an action functional defined on a space of unbased, contractible loops on a symplectic manifold together with an almost complex structure on the manifold. In this last example, the "flow lines" to be counted are deformed pseudo-holomorphic cylinders. In this introduction we shall denote any complex of one of these three types by $\mathcal{C}(f, \alpha)$.

Our focus in this paper is not on the homology computed by $\mathcal{C}(f, \alpha)$ (which, indeed, is in all these cases independent of the pair $(f, \alpha))$ but rather on the complexes themselves.

The first type of result that we prove for the Morse, Novikov as well as for the Floer complex is a rigidity statement: it shows that small $C^{0}$ perturbations of $f$ accompanied by arbitrary perturbations of $\alpha$ do not decrease the complexity of $\mathcal{C}(f, \alpha)$. Precisely, for fixed $(f, \alpha)$ there exists $\delta_{f}>0$ such that if $\left(f^{\prime}, \alpha^{\prime}\right)$ is another generic pair such that $\mathcal{C}\left(f^{\prime}, \alpha^{\prime}\right)$ is defined and with $\left\|f-f^{\prime}\right\|_{0} \leq \delta_{f}$, then $\mathcal{C}(f, \alpha)$ is a retract of $\mathcal{C}\left(f^{\prime}, \alpha^{\prime}\right)$ (which means that there are chain morphisms $i: \mathcal{C}(f, \alpha) \rightarrow \mathcal{C}\left(f^{\prime}, \alpha^{\prime}\right), j: \mathcal{C}\left(f^{\prime}, \alpha^{\prime}\right) \rightarrow \mathcal{C}(f, \alpha)$ such that $\left.j \circ i=i d\right)$. When $f=f^{\prime}$ we immediately deduce that the isomorphism type of $\mathcal{C}(f, \alpha)$ is independent of $\alpha$.

For the classical Morse complex and for the Novikov complex, this corollary is implicit in the work of Latour [12] where the approach is that of bifurcation analysis. The general case, when $f^{\prime}$ is different from (but $C^{0}$-close to) $f$, is new even in the standard Morse case and bifurcation analysis does not suffice to prove it. The reason is that $C^{0}$-closeness to $f$ is not naturally encoded in some restriction of the type of modification that may occur in the Morse complex at passage through bifurcation points when tracing a generic path of (of $C^{\infty}$ functions) from $f$ to $f^{\prime}$. Of course, without the $C^{0}$-closeness condition the result fails. Moreover, in the Floer setting bifurcation theory is very little understood and therefore particularly inefficient for this type of problem.

In this paper we do not use bifurcation analysis at all. Rather, we introduce and apply a notion of Morse cobordism. Roughly speaking, this is a generic pair $(f, \alpha)$ with $f$ a Morse function and $\alpha$ a Riemannian metric both defined on a cobordism $\left(M ; N_{0}, N_{1}\right)$ and such that $\nabla^{\alpha} f$ is tangent to the boundary and the restrictions $f_{i}=\left.f\right|_{N_{i}}$ are Morse functions whose critical points verify $i_{f_{i}}(x)=\operatorname{ind}_{f}(x)+i$, $i \in\{0,1\}$. The role of Morse cobordisms is to transform a $k$-parametric deformation question for Morse functions or complexes in a problem concerning a Morse function (or complex) defined on a space with $k$ more dimensions (in the paper the actual values of $k$ that are used are just 1 and 2). We introduce this notion in Sect. 1 and discuss its immediate properties in Sect. 1.1. In Sect. 1.2 we show the rigidity result for the Morse complex. This serves as prototype for the Floer and Novikov cases which are shown respectively in Sect. 2.1 and Sect. 2.2 Theorem 2.10 (i). It should be noted that the whole idea of Morse cobordisms is inspired by the continuation method in Floer theory. Indeed, the special Morse cobordisms without 
critical points in the interior of $M$ (which implies that $\left(M ; N_{0}, N_{1}\right)$ is a trivial cobordism) have an excellent Floer theory translation provided by the monotone homotopies introduced by Floer and Hofer in [5] (see also [2]) and which we shall use in Sect. 2.1.

In Sect. 1.3 we show a Mayer-Vietoris type formula for the Morse complex. A geometric cobordism $\left(M ; N_{0}, N_{1}\right)$ can be split along a two-sided hypersurface $N \subset \operatorname{Int}(M)$ into two cobordisms $\left(M^{\prime} ; N_{0}, N\right),\left(M^{\prime \prime} ; N, N_{1}\right)$. Of course, the initial cobordism can easily be recovered by gluing back the two pieces. We discuss how to split a Morse cobordism $f:\left(M ; N_{0}, N_{1}\right) \rightarrow \mathbb{R}$ along a Morse function $g: N \rightarrow \mathbb{R}$ into two Morse cobordisms $h^{\prime}: M^{\prime} \rightarrow \mathbb{R}$ and $h^{\prime \prime}: M^{\prime \prime} \rightarrow \mathbb{R}$. The key point showed in the gluing Theorem 1.29 , is that, if a certain technical property is satisfied by $g$, then it is possible to express in a purely algebraic manner the Morse complex of $f$ in terms of the Morse complexes of $h^{\prime}$ and $h^{\prime \prime}$. Again, for this result bifurcation methods are not directly usable (see Remark 1.30). Even if $g$ does not satisfy the technical property indicated the same method still produces the isomorphism type of the Morse complex of $f$.

The result in Sect. 1.3 is applied to the study of the Novikov complex in 2.2. In Theorem 2.10 (ii-iii) we describe a way to recover this complex algebraically out of information on one fundamental domain. For a fixed Morse-Smale function $(f, \alpha): M \rightarrow S^{1}$ the procedure consists in first splitting $f$ along a Morse function $g: N \rightarrow \mathbb{R}$ with $N$ a regular level surface of $f$. This produces a Morse cobordism on one fundamental domain of $f$. We then consider the Morse complex associated to this Morse cobordism and, by a purely algebraic procedure, we glue together an infinite number of copies of it thus getting a new complex $\mathcal{C}$. The problem then is to compare $\mathcal{C}$ with $\mathcal{C}(f, \alpha)$. By using again Morse cobordisms we show that these two complexes are isomorphic. The complex $\mathcal{C}$ is viewed as an approximation of $\mathcal{C}(f, \alpha)$ and special choices of $g$ result in a complex $\mathcal{C}$ agreeing with the Novikov complex of $f$ with any finite degree of precision desired. This last point is related to results of Pajitnov [16] which provide an exact formula for the Novikov complex but only apply to a special class of Morse-Smale functions. We discuss these relations in Remark 2.14(c).

\section{Cobordism of Morse functions}

1.1. Basic constructions. This subsection is concerned with the basic definitions and properties of Morse cobordisms.

1.1.1. Definitions. We start by introducing an extension of the usual notion of a Morse-Smale function on a closed manifold to a manifold with boundary. Our definition is designed to ensure that the standard construction of the chain complex in the closed case extends to a construction of a chain complex for a Morse-Smale function on a manifold with boundary.

Let $M$ be a compact manifold, possibly with non-empty boundary. A Morse function $f: M \rightarrow \mathbb{R}$ is a smooth function with nondegenerate critical points (that might belong to the boundary). We denote by $\operatorname{Crit}_{i}(f)\left(\operatorname{or}_{\mathrm{Crit}_{i}}(M, f)\right)$ the 
critical points $x$ of index $i$, $\operatorname{ind}_{f}(x)=i$, of such a function and we let $\mathrm{Crit}_{*}(f)$ (or $\left.\mathrm{Crit}_{*}(M, f)\right)$ be the set of all critical points of $f$.

Given a Morse function $f: M \rightarrow \mathbb{R}$ and a Riemannian metric $\alpha$ on $M$ let $\nabla f$ be the gradient vector field of $f$. Let $\phi=\left\{\phi_{t}: M \rightarrow M: t \in \mathbb{R}\right\}$ be the flow (possibly only partially defined) on $M$ induced by $-\nabla f$, so that

$$
d \phi_{t}(x) / d t=-\nabla f\left(\phi_{t}(x)\right), \quad \phi_{0}(x)=x \quad(x \in M) .
$$

For a critical point $p \in M$ of $f$ let

$$
W_{f}^{u}(p)=\left\{x \in M: \lim _{t \rightarrow-\infty} \phi_{t}(x)=p\right\}
$$

be the unstable manifold of $p$ and let

$$
W_{f}^{s}(p)=\left\{x \in M: \lim _{t \rightarrow \infty} \phi_{t}(x)=p\right\}
$$

be the stable manifold of $p$.

Definition 1.1. A Morse-Smale function $(f, \alpha)$ on a compact manifold with boundary $(M, \partial M)$ is a Morse function $f: M \rightarrow \mathbb{R}$ together with a Riemannian metric $\alpha$ such that

(i) for each boundary component $N$ of $\partial M$ one of the two following conditions is satisfied:

(a) $f$ is regular on $N$, meaning that for all $x \in N$

$$
\nabla f(x) \notin T_{x} N \subset T_{x} M .
$$

(b) For all $x \in N$ we have

$$
\nabla f(x) \in T_{x} N .
$$

(ii) for any two critical points $p, q \in M$ the stable and unstable manifolds $W^{u}(p)$ and $W^{s}(q)$ intersect transversely (due to point (i) the stable and unstable manifolds are indeed submanifolds of $M$, possibly with boundary if $\partial M \neq \emptyset$ ).

Remark 1.2. The classical definition of a Morse-Smale function assumes the boundary behaviour of (i) (a). Let $(g, \beta)=\left.(f, \alpha)\right|_{N}$ be defined when condition (i) (b) is satisfied. Then $(g, \beta)$ is also a Morse-Smale function on $N$. In this case, $\mathrm{Crit}_{*}(g) \subset \mathrm{Crit}_{*}(f)$ (because the component of $\nabla f(x)$ normal to the boundary is null for all $x \in N)$ and if $x \in \mathrm{Crit}_{*}(g)$ we have

$$
\operatorname{ind}_{g}(x)=\operatorname{ind}_{f}(x)-\delta_{x}
$$

with $\delta_{x} \in\{0,1\}$.

Let $(f, \alpha): M \rightarrow \mathbb{R}$ be Morse-Smale. Denote the set of critical points of $f$ with index $i$ by $\operatorname{Crit}_{i}(f)$. For $p \in \operatorname{Crit}_{i}(f), q \in \operatorname{Crit}_{j}(f)$ the space $Z(p, q)$ of (non-broken) flow lines of $\phi$ that join $p$ to $q$ is homeomorphic to any intersection $W^{u}(p) \cap W^{s}(q) \cap f^{-1}(a)$ where $a$ is some regular value of $f$ such that $f(q)<$ $a<f(p)$. The space 


$$
Z(p, q) \cong W^{u}(p) \cap W^{s}(q) \cap f^{-1}(a)
$$

is a $(i-j-1)$-dimensional manifold. If $j=i-1$ (and as $M$ is compact) this is a finite set. We fix orientations of $T_{x} M$ for all $x \in \mathrm{Crit}_{*}(f)$ as well as for each tangent space to the unstable manifolds $T_{x}\left(W^{u}(x)\right)$. This provides orientations for $T_{x}\left(W^{s}(x)\right)$ by demanding that the orientation on $T_{x}\left(W^{u}(x)\right)$ and that on $T_{x}\left(W^{s}(x)\right)$ give that fixed on $T_{x}(M)$. Moreover, as $W^{s}(x), W^{u}(x)$ are contractible these orientations induce orientations of the tangent spaces to the whole stable and unstable manifolds. For a point $z \in Z(p, q)$ we now let $\epsilon(z)$ be equal to 1 if the orientation of $T_{z}\left(W^{u}(p)\right)$ and that of $T_{z}\left(W^{s}(q)\right)$ (in this order) give the orientation induced on $T_{z} M$ from $T_{p}(M)$. Finally, let

$$
n^{f, \alpha}(p, q)=\sum_{z \in Z(p, q)} \epsilon(z) \in \mathbb{Z} .
$$

Definition 1.3. The $\mathbb{Z}$-coefficient Morse complex $C(M, f, \alpha)$ of a Morse-Smale function $(f, \alpha): M \rightarrow \mathbb{R}$ is defined by

$$
\begin{gathered}
d: C(M, f, \alpha)_{i}=\mathbb{Z}\left[\operatorname{Crit}_{i}(f)\right] \rightarrow C(M, f, \alpha)_{i-1}=\mathbb{Z}\left[\operatorname{Crit}_{i-1}(f)\right] ; \\
p \mapsto \sum_{q \in \operatorname{Crit}_{i-1}(f)} n^{f, \alpha}(p, q) q .
\end{gathered}
$$

The $\mathbb{Z}[\pi]$-coefficient Morse complex is defined for a Morse-Smale function $(f, \alpha): M \rightarrow \mathbb{R}$ and a regular cover $\widetilde{M}$ of $M$ with group of covering translations $\pi$. Let $(\widetilde{f}, \widetilde{\alpha}): \widetilde{M} \rightarrow \mathbb{R}$ be the pullback of $(f, \alpha)$, and let $\widetilde{\phi}$ be the pullback of $\phi$. The critical points $\widetilde{p} \in \widetilde{M}$ of $\widetilde{f}$ are the lifts of the critical points $p \in M$ of $f$. Fix two critical points $\widetilde{p} \in \operatorname{Crit}_{i}(\widetilde{f})$ and $\widetilde{q} \in \operatorname{Crit}_{j}(\widetilde{f})$. As each path in $M$ that originates at $p$ lifts to a unique path in $\widetilde{M}$ of origin $\widetilde{p}$, the space $\bigcup_{g \in \pi} Z(\widetilde{p}, g \widetilde{q})$ of flow lines of $\widetilde{\phi}$ that join $\widetilde{p}$ to one of the points $g \widetilde{q}, g \in \pi$ is homeomorphic to $Z(p, q)$. In particular, for $j=i-1$ the sum below is well defined and satisfies:

$$
\sum_{g \in \pi} n^{\tilde{f}, \widetilde{\alpha}}(\widetilde{p}, g \widetilde{q})=n^{f, \alpha}(p, q) \in \mathbb{Z}
$$

and

$$
n^{\tilde{f}, \widetilde{\alpha}}(g \tilde{p}, g \widetilde{q})=n^{\tilde{f}, \widetilde{\alpha}}(\widetilde{p}, \widetilde{q}) \in \mathbb{Z}(g \in \pi)
$$

Choose a lift of each critical point $p \in \operatorname{Crit}_{i}(f)$ to a critical point $\widetilde{p} \in \operatorname{Crit}_{i}(\widetilde{f})$, allowing the identification

$$
\operatorname{Crit}_{i}(\widetilde{f})=\pi \times \operatorname{Crit}_{i}(f) .
$$

Definition 1.4. The $\mathbb{Z}[\pi]$-coefficient Morse complex $C(M, f, \alpha)$ is the based f.g. free $\mathbb{Z}[\pi]$-module chain complex given by

$$
\begin{aligned}
d: C(M, f, \alpha)_{i}=\mathbb{Z}[\pi]\left[\operatorname{Crit}_{i}(f)\right] \rightarrow & C(M, f, \alpha)_{i-1}=\mathbb{Z}[\pi]\left[\operatorname{Crit}_{i-1}(f)\right] ; \\
p & \mapsto \sum_{q \in \operatorname{Crit}_{i-1}(f)}\left(\sum_{g \in \pi} n^{\widetilde{f}, \tilde{\alpha}}(\widetilde{p}, g \widetilde{q}) g\right) q .
\end{aligned}
$$


This clearly depends on the choices of the lifts $\tilde{p}$. More invariantly, the $\mathbb{Z}[\pi]-$ coefficient Morse complex can be written as

$$
\begin{array}{r}
d: C(M, f, \alpha)_{i}=\mathbb{Z}\left[\operatorname{Crit}_{i}(\widetilde{f})\right] \rightarrow C(M, f, \alpha)_{i-1}=\mathbb{Z}\left[\operatorname{Crit}_{i-1}(\widetilde{f})\right] ; \\
\widetilde{s} \mapsto \sum_{\widetilde{q} \in \operatorname{Crit}_{i-1}(\widetilde{f})} n^{\widetilde{f}, \widetilde{\alpha}}(\widetilde{s}, \widetilde{q}) \widetilde{q} .
\end{array}
$$

Remark 1.5. (a) In the classical situation

$$
(f, \alpha):\left(M ; N_{0}, N_{1}\right) \rightarrow([0,1] ;\{0\},\{1\})
$$

is a Morse-Smale function on a cobordism (Milnor [14]). This fits into our definition of a Morse-Smale function (1.1) provided that $f$ is regular on $N_{i}$ and its negative gradient points "out" on $N_{0}$ and "in" on $N_{1}$. Such a Morse-Smale function on an $m$-dimensional cobordism $\left(M ; N_{0}, N_{1}\right)$ determines a handle decomposition

$$
M=N_{0} \times[0,1] \cup \bigcup_{i=0}^{m} \bigcup_{\operatorname{Crit}_{i}(f)} D^{i} \times D^{m-i}
$$

The handle decomposition of $\left(M ; N_{0}, N_{1}\right)$ gives $\left(M, N_{0}\right)$ the structure of a relative $C W$ pair, with one $i$-cell for each $i$-handle. Franks' paper [7] identifies the Morse complex of $f$ with the associated cellular chain complex $C(M ; \mathbb{Z}[\pi])=C(\tilde{M})$. It is easy to verify that the definition gives a chain complex also in our more general context.

(b) Morse-Smale functions $f: M \rightarrow \mathbb{R}$ have the important property that if $p \in \mathrm{Crit}_{*}(f)$ and $\left\{x_{n}\right\} \subset W^{u}(p)$ is a convergent sequence in $M$ with limit $x_{\infty} \in M$, then $x_{\infty}$ is situated on a possibly broken flow line originating at $p$ (that is a flow line that passes geometrically through some other critical points besides $p$ before arriving in $x_{\infty}$ ). In fact, one of the proofs that the Morse complex is indeed a complex is obtained by understanding precisely the natural compactifications of the spaces of flow lines.

To simplify notation, we shall write $C(M, f)$ (resp. $\left.n^{f}(p, q)\right)$ instead of $C(M, f, \alpha)$ (resp. $\left.n^{f, \alpha}(p, q)\right)$ whenever the choice of metric $\alpha$ is clear from the context. Also, by an abuse of terminology, we shall write $C(M ; \mathbb{Z}[\pi])=C(\widetilde{M})$ as $C(M)$, and $H_{*}(M ; \mathbb{Z}[\pi])=H_{*}(\widetilde{M})$ as $H_{*}(M)$.

Definition 1.6. A cobordism from a Morse-Smale function

$$
\left(g_{1}, \beta_{1}\right): N_{1} \rightarrow \mathbb{R}
$$

to a Morse-Smale function

$$
\left(g_{0}, \beta_{0}\right): N_{0} \rightarrow \mathbb{R}
$$

is a cobordism $\left(M ; N_{0}, N_{1}\right)$ together with a Morse-Smale function

$$
(f, \alpha): M \rightarrow \mathbb{R}
$$

such that 
(i) $\left.(f, \alpha)\right|_{N_{0}}=\left(g_{0}, \beta_{0}\right)$ with

$$
\operatorname{ind}_{f}(p)=\operatorname{ind}_{g_{0}}(p)
$$

for each critical point $p \in N_{0}$,

(ii) $\left.(f, \alpha)\right|_{N_{1}}=\left(g_{1}, \beta_{1}\right)$ with

$$
\operatorname{ind}_{f}(p)=\operatorname{ind}_{g_{1}}(p)+1
$$

for each critical point $p \in N_{1}$.

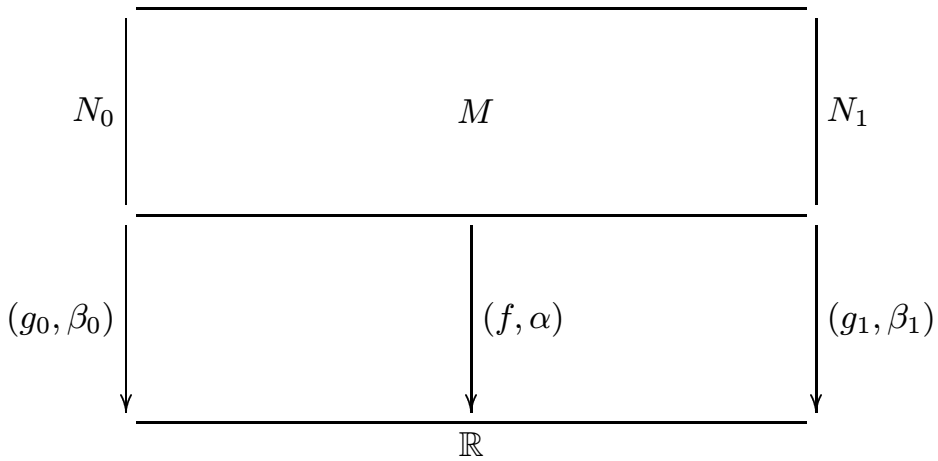

Remark 1.7. (a) We denote a Morse cobordism as before by

$$
(f, \alpha):\left(M ; N_{0}, N_{1}\right) \rightarrow \mathbb{R} .
$$

Clearly, $N_{0}$ and $N_{1}$ play different roles in the definition above. However, because we only work here with compact manifolds, it follows from Lemma 1.14 below that Morse-Smale functions $\left(g_{0}, \beta_{0}\right): N_{0} \rightarrow \mathbb{R},\left(g_{1}, \beta_{1}\right): N_{1} \rightarrow \mathbb{R}$ are cobordant if and only if the manifolds $N_{0}, N_{1}$ are cobordant and therefore the Morse-cobordism relation is an equivalence.

(b) The condition (i) in Definition 1.6 together with the Definition 1.1 imply that no negative gradient flow lines of $(f, \alpha)$ can leave $N_{0}$ towards $M \backslash \partial M$. Similarly, no trajectory can enter $N_{1}$ from the interior due to condition (ii).

Example 1.8. An $m$-dimensional cobordism $\left(M ; N_{0}, N_{1}\right)$ admits an embedding

$$
\left(M ; N_{0}, N_{1}\right) \subset \mathbb{R}^{n}
$$

for $n \geq 2 m+1$, with

$$
\begin{aligned}
& M \subset\left\{\left(x_{1}, x_{2}, \ldots, x_{n}\right) \in \mathbb{R}^{n}: 0 \leq x_{n} \leq 1\right\}, \\
& N_{0}=\left\{\left(x_{1}, x_{2}, \ldots, x_{n}\right) \in M: x_{n}=0\right\}, \\
& N_{1}=\left\{\left(x_{1}, x_{2}, \ldots, x_{n}\right) \in M: x_{n}=1\right\}
\end{aligned}
$$


and such that the height function

$$
f: M \rightarrow \mathbb{R} ;\left(x_{1}, x_{2}, \ldots, x_{n}\right) \mapsto x_{n}
$$

is Morse-Smale on $M \backslash \partial M$. As $f$ is constant on $N_{0}$ and $N_{1}$, it is not a cobordism of Morse-Smale functions. However, it is possible to slightly tilt $M$ inside $\mathbb{R}^{n}$ to obtain an isotopic embedding $\left(M ; N_{0}, N_{1}\right) \subset \mathbb{R}^{n}$ such that the height function $M \rightarrow \mathbb{R}$ restricts to Morse-Smale functions on $N_{0}$ and $N_{1}$. This is not yet a cobordism of Morse-Smale functions as in 1.6 because the component of the gradient of the height function that is normal to $\partial M$ does not generally vanish. As we shall see in Lemma 1.14 below, it is possible to perturb the height function in a neighbourhood of $\partial M$ such that the resulting function becomes a cobordism of Morse-Smale functions.

1.1.2. Immediate Properties of Morse cobordisms. We recall that the algebraic mapping cone $C(\phi)$ of a chain map $\phi: C \rightarrow D$ is the chain complex defined by

$$
d_{C(\phi)}=\left(\begin{array}{cc}
d_{D} & \phi \\
0 & -d_{C}
\end{array}\right): C(\phi)_{i}=D_{i} \oplus C_{i-1} \rightarrow C(\phi)_{i-1}=D_{i-1} \oplus C_{i-2} .
$$

A chain homotopy $\psi: \phi \simeq \phi^{\prime}: C \rightarrow D$ determines an isomorphism of the algebraic mapping cones

$$
h=\left(\begin{array}{ll}
1 & \psi \\
0 & 1
\end{array}\right): C(\phi) \rightarrow C\left(\phi^{\prime}\right) .
$$

If $C, D$ are based f.g. free $\mathbb{Z}[\pi]$-module chain complexes (as will be the case in the applications to Morse complexes) then $h$ is a simple isomorphism of based f.g. free $\mathbb{Z}[\pi]$-module chain complexes.

The Morse complex of a cobordism of Morse-Smale functions has the following homological properties:

Proposition 1.9. Let $(f, \alpha):\left(M ; N, N^{\prime}\right) \rightarrow \mathbb{R}$ be a cobordism of Morse-Smale functions $(g, \beta): N \rightarrow \mathbb{R},\left(g^{\prime}, \beta^{\prime}\right): N^{\prime} \rightarrow \mathbb{R}$, write

$$
D=C(N, g), \quad D^{\prime}=C\left(N^{\prime}, g^{\prime}\right), \quad F=C(M \backslash \partial M, f \mid),
$$

and let $\tilde{M}$ be a regular cover of $M$ with group of covering translations $\pi$.

(i) There are 3 types of critical points of $f$

$$
\operatorname{Crit}_{i}(M, f)=\operatorname{Crit}_{i}(N, g) \cup \operatorname{Crit}_{i}(M \backslash \partial M, f \mid) \cup \operatorname{Crit}_{i-1}\left(N^{\prime}, g^{\prime}\right) .
$$

The Morse complex of $f$ is given by

$$
\begin{aligned}
& C(M, f)_{i}=\mathbb{Z}[\pi]\left[\operatorname{Crit}_{i}(M, f)\right]=D_{i} \oplus F_{i} \oplus D_{i-1}^{\prime}, \\
& d_{C(M, f)}=\left(\begin{array}{ccc}
d_{D} & \theta & -\psi \\
0 & d_{F} & -\theta^{\prime} \\
0 & 0 & -d_{D^{\prime}}
\end{array}\right): \\
& C(M, f)_{i}=D_{i} \oplus F_{i} \oplus D_{i-1}^{\prime} \rightarrow C(M, f)_{i-1}=D_{i-1} \oplus F_{i-1} \oplus D_{i-2}^{\prime}
\end{aligned}
$$


with

$$
\begin{aligned}
& \theta: F_{i} \rightarrow D_{i-1} ; p \mapsto \sum_{q \in \mathrm{Crit}_{i-1}(g)} n^{f, \alpha}(p, q) q, \\
& \theta^{\prime}: D_{i}^{\prime} \rightarrow F_{i} ; p \mapsto \sum_{q \in \mathrm{Crit}_{i}(e)} n^{f, \alpha}(p, q) q, \\
& \psi: D_{i}^{\prime} \rightarrow D_{i} ; p \mapsto \sum_{q \in \operatorname{Crit}_{i}(g)} n^{f, \alpha}(p, q) q
\end{aligned}
$$

such that

$$
\begin{aligned}
& d_{D} \theta+\theta d_{F}=0: F_{i} \rightarrow D_{i-2}, \\
& d_{F} \theta^{\prime}-\theta^{\prime} d_{D^{\prime}}=0: D_{i}^{\prime} \rightarrow F_{i-1}, \\
& d_{D} \psi+\psi d_{D^{\prime}}+\theta \theta^{\prime}=0: D_{i}^{\prime} \rightarrow D_{i-1} .
\end{aligned}
$$

(ii) The Morse complex $C(M, f)$ is simple chain equivalent to $C\left(M, N^{\prime}\right)$, the $\mathbb{Z}[\pi]$-coefficient cellular complex for any relative $C W$ structure on $\left(M, N^{\prime}\right)$, with homology

$$
H_{*}(C(M, f))=H_{*}\left(M, N^{\prime}\right) .
$$

(iii) The subcomplex of $C(M, f)$

$$
C\left(M \backslash N^{\prime}, f \mid\right)=C(\theta)=\left(D_{i} \oplus F_{i},\left(\begin{array}{cc}
d_{D} & \theta \\
0 & d_{F}
\end{array}\right)\right)
$$

is simple chain equivalent to $C(M)$, with homology

$$
H_{*}\left(C\left(M \backslash N^{\prime}, f \mid\right)\right)=H_{*}(M) .
$$

(iv) The quotient complex of $C(M, f)$

$$
C(M \backslash N, f \mid)=C\left(\theta^{\prime}\right)=\left(F_{i} \oplus D_{i-1}^{\prime},\left(\begin{array}{cc}
d_{F} & -\theta^{\prime} \\
0 & -d_{D^{\prime}}
\end{array}\right)\right)
$$

is simple chain equivalent to $C(M, \partial M)$, with homology

$$
H_{*}(C(M \backslash N, f \mid))=H_{*}(M, \partial M) .
$$

(v) The subquotient complex of $C(M, f)$

$$
C(M \backslash \partial M, f \mid)=F
$$

is simple chain equivalent to $C(M, N)$, with homology

$$
H_{*}(C(M \backslash \partial M, f \mid))=H_{*}(F)=H_{*}(M, N) .
$$

(vi) The chain maps (up to sign)

$$
\begin{aligned}
& \theta: F=C(M \backslash \partial M, f \mid) \rightarrow D_{*-1}=C(N, g)_{*-1}, \\
& \theta^{\prime}: D^{\prime}=C\left(N^{\prime}, g^{\prime}\right) \rightarrow F=C(M \backslash \partial M, f \mid)
\end{aligned}
$$


induce the natural morphisms in homology

$$
\begin{aligned}
& \theta_{*}=\partial: H_{*}(F)=H_{*}(M, N) \rightarrow H_{*-1}(D)=H_{*-1}(N), \\
& \theta_{*}^{\prime}: H_{*}\left(D^{\prime}\right)=H_{*}\left(N^{\prime}\right) \rightarrow H_{*}(F)=H_{*}(M, N)
\end{aligned}
$$

and $\psi$ is a chain homotopy

$$
\psi: \theta \theta^{\prime} \simeq 0: D^{\prime}=C\left(N^{\prime}, g^{\prime}\right) \rightarrow D_{*-1}=C(N, g)_{*-1} .
$$

Proof. (i) For any $x \in N$

$$
\lim _{t \rightarrow \infty} \phi_{t}(x) \in N
$$

corresponding to the entry $d_{D}$ in the first column of $d_{C(M, f)}$.

For any $x \in M \backslash \partial M$

$$
\lim _{t \rightarrow \infty} \phi_{t}(x) \in N \text { or } M \backslash \partial M,
$$

corresponding to the two entries $\theta, d_{F}$ in the second column of $d_{C(M, f)}$.

For any $x \in N^{\prime}$

$$
\lim _{t \rightarrow \infty} \phi_{t}(x) \in N^{\prime} \text { or } M \backslash \partial M \text { or } N,
$$

corresponding to the three entries $-\psi,-\theta^{\prime},-d_{D^{\prime}}$ in the third column of $d_{C(M, f)}$. (ii) Slightly extend $M$ to a manifold $M^{\prime}$ by pasting to $M$ collars homeomorphic to $N \times[0,1]$ and $N^{\prime} \times[0,1]$. We also extend the function $f$ and the metric $\alpha$ to a function $f^{\prime}$ respectively a metric $\alpha^{\prime}$ in such a way that $f^{\prime}$ has the same critical points as $f$ and is regular and its gradient points out on $N^{\prime} \times\{1\}$ and points inside and is regular on $N \times\{1\}$. Then, by standard Morse theory, $C\left(M, f^{\prime}, \alpha^{\prime}\right)$ is simple chain equivalent to $C\left(M^{\prime}, N^{\prime}\right)$. On the other hand we have $C\left(M, f^{\prime}, \alpha^{\prime}\right)=C(M, f, \alpha)$.

(iii) The projection from $C(M, f)$ to $C\left(N^{\prime}, g^{\prime}\right)_{*-1}$ is given by

$$
C(M, f) \simeq C\left(M, N^{\prime}\right) \stackrel{\partial}{\longrightarrow} C\left(N^{\prime}, g^{\prime}\right)_{*-1} \simeq C\left(N^{\prime}\right)_{*-1}
$$

The kernel of this projection is precisely $C\left(M \backslash N^{\prime}, f \mid\right)$ and this complex is therefore simple chain equivalent to $C(M)$.

(iv) The chain inclusion

$$
C(N, g) \simeq C(N) \rightarrow C(M, f) \simeq C\left(M, N^{\prime}\right)
$$

is a chain representative of the inclusion $N \hookrightarrow\left(M, N^{\prime}\right)$. Therefore, $C(M \backslash N, f \mid)$ (which is the co-kernel of the map above) is simple chain equivalent to $C\left(M, N \cup N^{\prime}\right)$ $=C(M, \partial M)$.

(v) The chain inclusion

$$
C(N, g) \simeq C(N) \rightarrow C\left(M \backslash N^{\prime}, f \mid\right) \simeq C(M)
$$

is a chain representative of the inclusion $N \hookrightarrow M$.

(vi) Combine (ii), (iii), (iv) and (v). 
A particular type of Morse cobordism will play an important role further on.

Definition 1.10. A cobordism of Morse-Smale functions

$$
(f, \alpha):\left(M ; N, N^{\prime}\right) \rightarrow \mathbb{R}
$$

is simple if $f$ does not have any critical points in $M \backslash \partial M$, so that

$$
\operatorname{Crit}_{i}(M, f)=\operatorname{Crit}_{i}(N, g) \cup \operatorname{Crit}_{i-1}\left(N^{\prime}, g^{\prime}\right) .
$$

Proposition 1.11. Let $(f, \alpha):\left(M ; N, N^{\prime}\right) \rightarrow \mathbb{R}$ be a simple cobordism of MorseSmale functions $(g, \beta): N \rightarrow \mathbb{R},\left(g^{\prime}, \beta^{\prime}\right): N^{\prime} \rightarrow \mathbb{R}$.

(i) The identity $N \rightarrow N$ extends to a diffeomorphism

$$
\left(M ; N, N^{\prime}\right) \rightarrow N \times([0,1] ;\{0\},\{1\}) .
$$

(ii) The inclusions $N \hookrightarrow M, N^{\prime} \hookrightarrow M$ induce simple chain equivalences

$$
C(N, g) \simeq C(M), C\left(N^{\prime}, g^{\prime}\right) \simeq C(M)
$$

with $C(M)$ the $\mathbb{Z}[\pi]$-coefficient cellular chain complex of any $C W$ structure on $M$. (iii) The chain map

$$
f^{M}: C\left(N^{\prime}, g^{\prime}\right) \rightarrow C(N, g)
$$

defined by

$$
\begin{aligned}
f^{M}: C\left(N^{\prime}, g^{\prime}\right)_{i}=\mathbb{Z}[\pi]\left[\operatorname{Crit}_{i}\left(g^{\prime}\right)\right] & \rightarrow C(N, g)_{i}=\mathbb{Z}[\pi]\left[\mathrm{Crit}_{i}(g)\right] ; \\
x & \mapsto \sum_{y} n^{f}(x, y) y
\end{aligned}
$$

is a simple chain equivalence, with $n^{f}(x, y)$ the algebraic number of downward gradient flow lines in $\widetilde{M}$ from $x$ to $y$, for any critical points $x \in \widetilde{N}^{\prime}, y \in \widetilde{N}$ with

$$
\operatorname{ind}_{g^{\prime}}(x)=\operatorname{ind}_{f}(x)-1=\operatorname{ind}_{g}(y)=\operatorname{ind}_{f}(y)=i .
$$

Proof. (i) It is possible to find a small perturbation of the negative gradient flow of $f$ in a neighbourhood of $\partial M$ such that the resulting flow $\gamma^{\prime}$ points inside $M$ on $N^{\prime}$ and outside on $N$; it is gradient like and has no stationary points inside $M$. The existence of such a flow implies the claim. To construct this perturbation fix some Morse charts $U_{i}$ around the critical points of $f$ that are situated on $N$. Fix also a collared neighbourhood of $N$ that is diffeomorphic to $N \times[0, \epsilon]$. With respect to this parametrization notice that, for all small enough $\delta$ the projection of $\nabla f(x)$ on $T_{x}(N \times\{\delta\})$ is non-zero for all sufficiently small $\delta$ and $x \notin \cup U_{i}$. Moreover, for $\delta$ small and $x \in U_{i}$ we have that $\nabla f(x)$ is not tangent to $T_{x}(N \times\{\delta\})$ and points towards $N \times\{0\}=N$ (because of the Morse lemma). This means that by adding to $-\nabla f$ a vector field which is 0 outside $N \times[0, \tau]$ ( $\tau$ small enough) and is equal to $-h(t) \partial / \partial t$ for $x=(z, t) \in N \times[0, \tau]$ with $h:[0, \tau] \rightarrow[0,1]$ decreasing, $h(0)=1, h(\tau)=0$ the induced flow will satisfy the desired properties with respect to $N$. Of course, a similar construction is possible relative to $N^{\prime}$ and produces $\gamma^{\prime}$. 
(ii) Immediate from (i).

(iii) The chain map $f^{M}$ is the chain homotopy of Proposition 1.9 (vi)

$$
\psi=f^{M}: 0 \simeq 0: C\left(N^{\prime}, g^{\prime}\right) \rightarrow C(N, g)_{*-1} .
$$

For any $C W$ structure on $M$ the chain map $f^{M}$ is chain homotopic to the composite of the simple chain equivalences given by (ii)

$$
C\left(N^{\prime}, g^{\prime}\right) \rightarrow C(M) \rightarrow C(N, g) .
$$

1.1.3. Construction of Morse-Smale cobordisms. We shall discuss here a few simple ways to construct Morse cobordisms. We first fix some more notation. For $i=0,1,2, \ldots$ let $C^{i}(N, \mathbb{R})$ be the space of $C^{i}$-functions $h: N \rightarrow \mathbb{R}$, with the following topology. For $i=0$ use the norm

$$
\|h\|_{0}=\sup \{|h(x)|: x \in N\} .
$$

For $i \geq 1$ use the Whitney $C^{i}$-topology, in which a neighbourhood of $f \in C^{i}(N, \mathbb{R})$ consists of those $g \in C^{i}(N, \mathbb{R})$ such that in local coordinates, $f$ and $g$ together with their first $i$ derivatives are within $\epsilon$ at each point of $N$ (Hirsch [8], p. 35).

Here is a result that summarizes the output of some of our constructions. All the arguments here are simple once the correct statements are formulated - similar constructions have been independently used by Abbondandolo and Majer [1]. We include the details because we shall use these constructions repeatedly later in the paper.

Proposition 1.12. Fix a Morse-Smale function $(h, \gamma)$ on a compact closed manifold $N$. Consider $\left(h^{\prime}, \gamma^{\prime}\right): N \rightarrow \mathbb{R}$, another Morse-Smale function on $N$, such that $h(x)>h^{\prime}(x), \forall x \in N$. There exists a simple Morse-Smale cobordism $(H, \Gamma): N \times[0,1] \rightarrow \mathbb{R}$ from $(h, \gamma)$ to $\left(h^{\prime}, \gamma^{\prime}\right)$ such that

$$
h^{\prime}(x) \leq H_{t}(x) \leq h(x) \quad(x \in N, t \in[0,1]) .
$$

The resulting chain map

$$
i=H^{N \times[0,1]}: C(N, h, \gamma) \rightarrow C\left(N, h^{\prime}, \gamma^{\prime}\right)
$$

is a simple chain equivalence.

Proof. The proof is an immediate consequence of the constructions of Morse cobordisms described in the lemmas below.

Lemma 1.13. Let $\left(f_{0}, \alpha_{0}\right)$ be a Morse-Smale function on a closed manifold $N$. Let $c: N \rightarrow \mathbb{R}$ be such that $c(x)>f_{0}(x)$ for all $x \in N$ and let $\alpha_{1}$ be a second metric on $N$. There is a Morse-Smale function $(f, \alpha)$ on $N \times[0,1]$ such that $\left.f\right|_{N_{0}}=f_{0},\left.f\right|_{N_{1}}=c$, $\alpha$ extends the $\alpha_{i}$ 's and $\partial f / \partial t(x, t)>0$ for all $x \in N, t>0$ $\left(N_{i}=N \times\{i\}\right)$. 
Proof. Let $u:[0,1] \rightarrow[0,1]$ be a $C^{\infty}$ function such that

$$
u(0)=1, u(1)=0, u^{\prime}(0)=0, u^{\prime}(t)<0(t>0), u^{\prime \prime}(0)<0 .
$$

Consider the function

$$
f(x)=u(t) f_{0}(x)+(1-u(t)) c(x) .
$$

We have

$$
\begin{aligned}
& \partial f / \partial t=u^{\prime}(t)\left(f_{0}(x)-c(x)\right), \\
& \partial f / \partial x=u(t) \partial f / \partial x+(1-u(t)) \partial c / \partial x
\end{aligned}
$$

and this together with the fact that

$$
\begin{aligned}
& \left(\partial^{2} f / \partial x^{2}\right)(x, 0)=\left(\partial^{2} f_{0} / \partial x^{2}\right)(x), \\
& \left(\partial^{2} f / \partial t^{2}\right)(x, 0)=u^{\prime \prime}(t)\left(f_{0}(x)-c(x)\right)
\end{aligned}
$$

implies immediately the statement (notice that $f$ is regular on $N_{1}$ ), except that we also need to remark that there is a metric $\alpha$ extending the $\alpha_{i}$ 's such that $f$ is Morse-Smale with respect to $\alpha$. By using a partition of unity argument it follows that there is a metric $\alpha^{\prime}$ that extends the $\alpha_{i}$ 's. Moreover, as $\left(f_{0}, \alpha_{0}\right)$ is already a Morse-Smale function we can slightly modify $\alpha^{\prime}$ away from $\partial(N \times[0,1])$ to obtain $\alpha$.

Lemma 1.14. Consider a compact cobordism $\left(M ; N_{0}, N_{1}\right)$, and suppose given Morse-Smale functions $\left(f_{i}, \alpha_{i}\right): N_{i} \rightarrow \mathbb{R}(i=0,1)$. Let $g: M \rightarrow \mathbb{R}$ be a Morse function with all its critical points in the interior of $M$ and which is constant, maximal on $N_{1}$, and constant, minimal on $N_{0}$. For any neighbourhood $W$ of $\partial M$ there are suitable constants $c_{0}, c_{1}$ and a Morse-Smale cobordism $(f, \alpha):\left(M ; N_{0}, N_{1}\right) \rightarrow \mathbb{R}$ between the Morse-Smale functions $\left(f_{0}+c_{0}, \alpha_{0}\right)$ and $\left(f_{1}+c_{1}, \alpha_{1}\right)$ such that

$$
f|=g|: M \backslash W \rightarrow \mathbb{R} .
$$

Proof. Inside the neighbourhood $W$ of $\partial M$ we can find a tubular neighbourhood that we shall identify with $N_{0} \times[0,1] \coprod N_{1} \times[0,1]$. We may assume that $\partial M=$ $N_{0} \times\{0\} \coprod N_{1} \times\{1\}$ and that $g$ is constant, regular and equal to $k_{0}$ on $N_{0} \times\{1\}$ and is constant, regular and equal to $k_{1}$ on $N_{1} \times\{0\}$. Pick $c_{i} \in \mathbb{R}$ such that $f_{0}(x)+c_{0}<k_{0}$ and $f_{1}(x)+c_{1}>k_{1}$. Now Lemma 1.13 provides a cobordism between $f_{0}+c_{0}$ and the constant function $k_{0}$ on $N_{0} \times[0,1]$. By the same method as in 1.13 we also get an analogous cobordism between $k_{1}$ and $f_{1}+c_{1}$ on $N_{1} \times[0,1]$. Another partition of unity argument shows that these two cobordisms can be pasted together with $g$ to provide the function $f$. The metric $\alpha$ is obtained by the same argument as in 1.13 .

Remark 1.15. For the cobordism $(f, \alpha)$ constructed in 1.14 the chain complex $C(M \backslash \partial M, f)$ of Proposition 1.9 coincides with $C(M, g)$. 
Definition 1.16. Let $\left(f_{0}, \alpha_{0}\right),\left(f_{1}, \alpha_{1}\right): N \rightarrow \mathbb{R}$ be Morse-Smale functions on a closed manifold $N$ such that

$$
f_{1}(x)>f_{0}(x)(x \in N) .
$$

A linear cobordism between $\left(f_{0}, \alpha_{0}\right)$ and $\left(f_{1}, \alpha_{1}\right)$ is a simple cobordism (1.10)

$$
(f, \alpha): N \times([0,1] ;\{0\},\{1\}) \rightarrow \mathbb{R}
$$

with $\left.(f, \alpha)\right|_{N \times\{i\}}=\left(f_{i}, \alpha_{i}\right)(i=0,1)$ such that for all $t \in[0,1], f_{t}$ is a convex, linear combination of $f_{0}$ and $f_{1}$ and $\partial f / \partial t>0$ for all points $(x, t) \in N \times(0,1)$.

Lemma 1.17. Any two Morse-Smale functions $\left(f_{0}, \alpha_{0}\right)$, $\left(f_{1}, \alpha_{1}\right)$ on a closed manifold $N$ such that

$$
f_{1}(x)>f_{0}(x) \quad(x \in N)
$$

are related by a linear cobordism. If $\alpha_{0}=\alpha_{1}$ there is a simple cobordism $(f, \alpha)$ with $\alpha=\alpha_{0}+d t^{2}$ the product metric. If moreover $f_{1}=f_{0}+c$ with $c>0$ constant, then there exists a linear cobordism with $\alpha$ the product metric and for any such linear cobordism the chain map $f^{N \times[0,1]}: C\left(N, f_{1}\right) \rightarrow C\left(N, f_{0}\right)$ is a simple isomorphism.

Proof. As in the proof of Lemma 1.13 we consider a $C^{\infty}$ function $v:[0,1] \rightarrow$ $[0,1]$ such that

$$
\begin{aligned}
& v(0)=1, v(1)=0, v^{\prime}(1)=v^{\prime}(0)=0, \\
& v^{\prime}(t)<0(0<t<1), v^{\prime \prime}(0)<0<v^{\prime \prime}(1) .
\end{aligned}
$$

We define

$$
f(x)=v(t) f_{0}(x)+(1-v(t)) f_{1}
$$

with the immediate consequence that, as above, one can find a metric $\alpha$ such that the Morse-Smale function $(f, \alpha)$ satisfies the properties required in the first part of the statement. For the second part, assume that $\alpha$ is the product metric $\alpha_{0}+d t^{2}$. As both $\left(f_{0}, \alpha_{0}\right),\left(f_{1}, \alpha_{1}\right)$ are Morse-Smale we can modify $f$ outside a neighbourhood of $\partial(N \times[0,1])$ to obtain the desired Morse-Smale cobordism. If $f_{1}=f_{0}+c$ this modification is not necessary, because the function $f$ itself is already MorseSmale with respect to $\alpha$. Moreover, if $x \in \operatorname{Crit}\left(f_{0}\right)$ then for all $t \in[0,1]$ the flow induced by $-\nabla f$ is tangent to $x \times[0,1]$. As $\operatorname{ind}_{f}(x \times\{0\})=\operatorname{ind}_{f_{0}}(x)$, ind $_{f}(x \times\{1\})=\operatorname{ind}_{f_{0}}(x)+1$ the proof is concluded.

The proof of Proposition 1.12 follows by applying the construction in Lemma 1.17 to the Morse-Smale functions $\left(f_{0}, \alpha_{0}\right)=\left(h^{\prime}, \gamma^{\prime}\right)$ and $\left(f_{1}, \alpha_{1}\right)=(h, \gamma)$.

Remark 1.18. Note that if $(g, \beta)$ is a Morse-Smale function on $N$, then the Morse complexes $C(N, g, \beta)$ and $C(N, c(g+k), \beta)$, where $c, k \in \mathbb{R}$ are constants, are canonically identified. This implies that, on a compact manifold, we may use Proposition 1.12 to compare the Morse complexes of any two Morse functions $h$ and $h^{\prime}$ by simply adding to $h$ a sufficiently large constant $S$ such that $h(x)+S>h^{\prime}(x)$, $\forall x \in N$. 
1.2. Rigidity of the Morse complex. We now state and prove the Rigidity Theorem 1.19 for Morse complexes.

Fix a Morse-Smale function $(h, \gamma)$ on a compact closed manifold $N$. Consider $\left(h^{\prime}, \gamma^{\prime}\right): N \rightarrow \mathbb{R}$, another Morse-Smale function on $N$, and let $S>0$ be such that $S>\left\|h^{\prime}-h\right\|_{0}$. By Proposition 1.12 and Remark 1.18 there exists a simple Morse-Smale cobordism $(H, \Gamma): N \times[0,1] \rightarrow \mathbb{R}$ from $(h+S, \gamma)$ to $\left(h^{\prime}, \gamma^{\prime}\right)$ such that

$$
h^{\prime}(x) \leq H_{t}(x) \leq h(x)+S(x \in N, t \in[0,1])
$$

and the induced chain map

$$
i(H)=H^{N \times[0,1]}: C(N, h, \gamma) \rightarrow C\left(N, h^{\prime}, \gamma^{\prime}\right)
$$

is a simple chain equivalence.

Rigidity Theorem 1.19. In the setting above let

$$
\delta=\min \left\{|h(x)-h(y)|: x, y \in \mathrm{Crit}_{*}(h), W^{u}(x) \bigcap W^{s}(y) \neq \emptyset\right\} .
$$

(i) If $S<\delta / 2$, then there exists a chain map j:C(N, $\left.h^{\prime}, \gamma^{\prime}\right) \rightarrow C(N, h, \gamma)$ such that

$$
j \circ i(H)=\text { identity }: C(N, h, \gamma) \rightarrow C(N, h, \gamma) \text {. }
$$

(ii) There exists a $C^{2}$-neighbourhood of $h, \mathcal{U}_{h}$, such that if $h^{\prime} \in \mathcal{U}_{h}$ then the simple chain equivalence $i(H)$ is a simple isomorphism.

Remark 1.20. (a) The existence of a constant $S$ satisfying condition (i) is implied by the assumption $\left\|h-h^{\prime}\right\|_{0}<\delta / 2$. This means that whenever this last condition is satisfied the Morse complex of $h$ is a retract of the Morse complex of $h^{\prime}$. Moreover, this relation is independent of the metrics used in the definition of these complexes. This also implies that the number of critical points of index $k$ of $h^{\prime}$ is at least that of $h$. It should be noted that $h$ and $h^{\prime}$ do not play symmetric roles in the statement, since $\delta$ depends on $h$. One of the most striking features of (i) is that only the closeness of $h^{\prime}$ to $h$ in the $C^{0}$ norm is required for the relation between the Morse complexes of $h$ and $h^{\prime}$ to hold. This allows $h^{\prime}$ to have a different number of critical points than $h$ and, in particular, one could apply (i) to a function $h^{\prime}$ obtained from $h$ by a process analogous to the subdivision of a cell decomposition of a $C W$ complex.

(b) Even if $f_{0}=f_{1}$ it is easy to produce examples such that the isomorphism at

(ii) is not equal to the identity.

(c) The condition $S<\delta / 2$ appears to be optimal. It is certainly essential for the proof we shall present below.

In Sect. 1.2 we prove 1.19 (i), and deduce 1.19 (ii) as an immediate consequence. The key new idea appears in the proof of (i) and is as follows. As Morse-Smale cobordisms are themselves Morse functions we may apply the constructions in 
Sect. 1.1.3 to cobordisms. This leads in Lemma 1.21 to a Morse function $g$ defined on $N \times([0,1] \times[0,1])$ having critical points only for the second coordinate belonging to the corners of the square $[0,1] \times[0,1]$. The function $g$ restricts to $h+$ convenient constants in the corners $(00),(01),(11)$ and to $h^{\prime}+$ some constant in the corner (10). The critical points of $g$ in the corner $(i j)$ are those of $h$ (resp. $h^{\prime}$ ) but of index raised by $i+j$. Moreover, the simple cobordism $H$ (+ some constant) appears on the side (10)(11). On the sides (00)(01) and (01)(11) we have a certain type of trivial simple cobordism that induces the identity in terms of Morse complexes. The purpose is to show that the restriction of $g$ on the sides (10)(11) and (00)(10) induce morphisms whose composition $A$ is an isomorphism. For this we compare this composition with that of the morphisms appearing on the sides (01)(11) and (00)(01) which, as said before, is the identity. The two compositions are measured by flow lines of $g$ that join critical points of indexes differing by 2 , that originate in (11) and end in (00), and are broken precisely once at one critical point of intermediate index that belongs to (10) for the first composition and to (01) for the second. If a flow line of $g$ originating at a critical point in (11) and reaching a critical point in (00) which is broken once would break necessarily in a point in (10) or (01), then the two compositions would be equal. This is not necessarily the case though: the breaking point can belong also to (00) or to (11). However, when the assumption on $S$ at (i) is assumed, such a flow line with the origin in a critical point of $x \in \mathrm{Crit}_{i}(h) \times(11)$ and ending at $y \in \mathrm{Crit}_{i}(h) \times(00)$ with $h(y) \geq h(x)$ can only break at a point in (01) or in (10) (by Lemma 1.22) essentially because along the negative-flow lines of $g$ the value of $g$ has to decrease and a break in a point of (00) or (11) would force the change in value of $g$ to be bigger than allowed by the fact that $h(y) \geq h(x)$. This is enough to show that our composition $A$ is an isomorphism even if it might not be the identity.

We now proceed to the actual proof of the Rigidity Theorem 1.19.

Proof. We first observe that the notion of Morse-Smale cobordism introduced in Definition 1.6 can be generalized in an obvious way to the case when $N_{0}$ and $N_{1}$ have boundaries and $f_{i}$ is tangent to $\partial N_{i}$ (in the sense that $\nabla f_{i}(x) \in T_{x} \partial N_{i}$ for all $\left.x \in \partial N_{i}, i=0,1\right)$.

All the previous statements have analogues in this case. In particular, the statements of 1.9, 1.11, 1.13, 1.14, 1.17 remain true when assuming that the manifold $N$ has a non-empty boundary. A general existence result for two-parameter MorseSmale functions follows.

Lemma 1.21. Let $W$ be a compact manifold without boundary. Let $\left(f_{j}, \alpha_{j}\right)$ be Morse-Smale functions on $W, j=0,1,2,3$ and let $\left(g_{i j}, \beta_{i j}\right)$ be simple Morse-Smale cobordisms on $W \times[0,1]$ of $\left(\alpha_{i}, f_{i}\right)$ and $\left(\alpha_{j}, f_{j}\right)$ for $(i, j) \in$ $\{(0,1),(0,2),(1,3),(2,3)\}$. Assume $g_{13}>g_{02}$ and suppose that $g_{01}$ and $g_{23}$ are linear cobordisms. There exists a simple Morse-Smale cobordism $(g, \beta)$ on $(W \times[0,1]) \times[0,1]$ of $\left(g_{02}, \beta_{02}\right)$ and $\left(g_{13}, \beta_{13}\right)$ which restricts to $\left(g_{0+2 k, 1+2 k}\right.$, $\left.\beta_{0+2 k, 1+2 k}\right)$ on $W \times\{k\} \times[0,1](k=0,1)$, with

$$
\operatorname{Crit}_{i}(g)=\operatorname{Crit}_{i-2}\left(f_{3}\right) \cup \operatorname{Crit}_{i-1}\left(f_{2}\right) \cup \operatorname{Crit}_{i-1}\left(f_{1}\right) \cup \operatorname{Crit}_{i}\left(f_{0}\right) .
$$


Proof. Let

$$
g_{01}=u(t) f_{0}+(1-u(t)) f_{1}, g_{23}=r(t) f_{2}+(1-r(t)) f_{3}
$$

with $u, r:[0,1] \rightarrow[0,1]$ functions with the properties described at the beginning of the proof of Lemma 1.17. Let $w:[0,1] \rightarrow[0,1]$ be another such function. Define

$$
\begin{aligned}
g(x, t, \tau)= & g_{02}(x, \tau)(u(t) w(\tau)+r(t)(1-w(\tau)) \\
& +g_{13}(x, \tau)((1-u(t)) w(\tau)+(1-r(t))(1-w(\tau))) .
\end{aligned}
$$

By the same type of argument as those used before it is easy to see that $g$ is a Morse function. Moreover, by a partition of unity argument one can construct a metric $\beta^{\prime}$ extending the $\beta_{i j}$ 's. A small perturbation of $\beta^{\prime}$ away from the boundary of $W \times[0,1] \times[0,1]$ leads to a new metric $\beta$ such that the pair $(\beta, g)$ satisfies the desired properties.

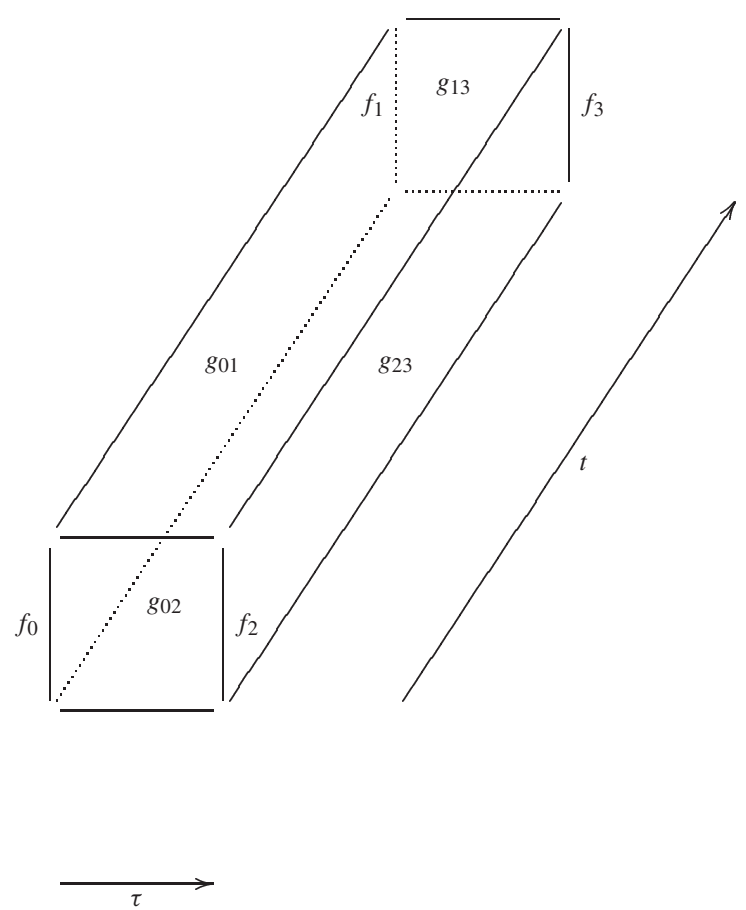

Lemma 1.22. In the setting of Lemma 1.21 above, there is an $\epsilon>0$ such that for any $u \in \mathrm{Crit}_{k-2}\left(f_{3}\right)$ and $v \in \mathrm{Crit}_{k-2}\left(f_{0}\right)$ with $f_{3}(u)-f_{0}(v)<\epsilon$ we have

$$
\sum_{x \in \mathrm{Crit}_{k-2}\left(f_{1}\right)} n^{g_{13}}(u, x) n^{g_{01}}(x, v)+\sum_{y \in \mathrm{Crit}_{k-2}\left(f_{2}\right)} n^{g_{23}}(u, y) n^{g_{02}}(y, v)=0 .
$$


Proof. For $i=0,3$, let

$$
\begin{aligned}
& \epsilon_{i}=\min \left\{f_{i}(a)-f_{i}(b): a \in \operatorname{Crit}_{k}\left(f_{i}\right),\right. \\
& \left.\quad b \in \operatorname{Crit}_{k-1}\left(f_{i}\right), W^{u}(a) \bigcap W^{s}(b) \neq \emptyset, k \in \mathbf{N}\right\} .
\end{aligned}
$$

As $W$ is compact $\epsilon_{i}>0$. Let $\mu>0$ be a small positive constant such that

$$
\epsilon=\min \left\{\epsilon_{0}, \epsilon_{3}\right\}-\mu>0 \text {. }
$$

As a consequence of the fact that $C(M, g)$ is a chain complex we have

$$
\begin{gathered}
\sum_{x \in \operatorname{Crit}_{k-2}\left(f_{1}\right)} n^{g_{13}}(u, x) n^{g_{01}}(x, v)+\sum_{y \in \operatorname{Crit}_{k-2}\left(f_{2}\right)} n^{g_{23}}(u, y) n^{g_{02}}(y, v)+ \\
\sum_{s \in \operatorname{Crit}_{k-1}\left(f_{0}\right)} n^{g}(u, s) n^{f_{0}}(s, v)+\sum_{l \in \operatorname{Crit}_{k-3}\left(f_{3}\right)} n^{f_{3}}(u, l) n^{g}(l, v)=0 .
\end{gathered}
$$

The condition imposed to $u$ and $v$ implies that the two last sums of this expression vanish. Indeed, in the sum before last the only terms that count are those that satisfy $f_{0}(v)<f_{0}(s)<f_{3}(u)$. This implies $f_{0}(s)-f_{0}(v)<\epsilon$. It follows that $n^{f_{0}}(s, v)=0$. The argument for the vanishing of the last sum is similar.

We now use Lemma 1.22 to prove the Rigidity Theorem 1.19 (i). We return to the setting of the statement of the theorem. Thus $N,(h, \gamma), \delta, S<\delta / 2$ and $\left(h^{\prime}, \gamma^{\prime}\right)$ are fixed as well as the simple Morse-Smale cobordism $(H, \Gamma)$ of $\left(h^{\prime}, \gamma^{\prime}\right)$ and $(h+S, \gamma)$. Let $\mu>0$ such that $S+\mu<\delta / 2$. We intend to use Lemmas 1.21 and 1.22. We take

$$
\begin{aligned}
& W=N,\left(f_{0}, \alpha_{0}\right)=(h, \gamma), \\
& \left(f_{1}, \alpha_{1}\right)=\left(h^{\prime}+S+\mu, \gamma^{\prime}\right),\left(f_{2}, \alpha_{2}\right)=(h+\mu, \gamma), \\
& \left(f_{3}, \alpha_{3}\right)=(h+2 S+\mu, \gamma),\left(g_{13}, \beta_{13}\right)=(H+S+\mu, \Gamma)
\end{aligned}
$$

and $\left(g_{02}, \beta_{02}\right)$ a linear Morse-Smale cobordism of $(h, \gamma)$ and $(h+\mu, \gamma)$ with $\beta_{02}$ a product metric. As we have $h^{\prime}(x)+S>h(x)$ for all $x \in N$ we note that

$$
g_{13}(x, \tau)=H(x, \tau)+S+\mu \geq h^{\prime}(x)+S+\mu>h(x)+\mu \geq g_{02}(x, \tau) .
$$

Therefore the conditions in Lemma 1.21 are verified. We may also take $\left(g_{23}, \beta_{23}\right)$ such that this is a linear cobordism with $\beta_{23}$ the product metric (see Lemma 1.17). We use Lemma 1.21 to construct $(g, \beta)$. Lemma 1.22 can now be applied and by inspecting its proof we see that we may take $\epsilon=\delta-\mu$. We fix a total order on the set $\mathrm{Crit}_{*}(h)=\mathrm{Crit}_{*}\left(f_{0}\right)=\mathrm{Crit}_{*}\left(f_{3}\right)$ such that for $x, y \in \mathrm{Crit}_{*}(h)$ the inequality $h(x) \leq h(y)$ implies $x \leq y$. With this total ordering we consider the matrix $A=\left(a_{i j}\right)$ of the composition $g_{01}^{N \times[0,1]} \circ g_{13}^{N \times[0,1]}$ as well as the matrix $B=\left(b_{i j}\right)$ of the composition $g_{02}^{N \times[0,1]} \circ g_{23}^{N \times[0,1]}$. Because both $g_{02}$ and $g_{23}$ are linear cobordisms with the product metric it immediately follows that $B=I d$. We now want to observe that $A$ is an upper triangular matrix with -1 's on the 
diagonal. Indeed, assume $x, y \in \mathrm{Crit}_{k}(h)$ correspond to elements $i, j$ respectively in the fixed order of $\mathrm{Crit}_{*}(h)$ with $i \leq j$. Then $f_{0}(x) \leq f_{0}(y)$ and

$$
f_{3}(x)-f_{0}(y)=f_{0}(x)+2 S+\mu-f_{0}(y)<\delta-\mu=\epsilon .
$$

Therefore, by Lemma 1.22 we have

$$
a_{i j}=\sum_{z \in \mathrm{Crit}_{k}\left(f_{1}\right)} n^{g_{13}}(x \times(1,1), z \times(0,1)) n^{g_{01}}(z \times(0,1), y \times(0,0))=-b_{i j}
$$

and as mentioned above $b_{i j}=\delta_{i j}$, the Kronecker symbol. As a consequence we get that $A$ has determinant equal to \pm 1 and is therefore an isomorphism. But $g_{13}^{N \times[0,1]}=H^{N \times[0,1]}$, and this completes the proof of the Rigidity Theorem 1.19 (i).

We now turn to 1.19 (ii). If $\mathcal{U}_{h}$ is sufficiently small, then the number of critical points of $h^{\prime} \in \mathcal{U}_{h}$ equals the number of critical points of $h$. Of course, we may assume $\mathcal{U}_{h}$ sufficiently small such that $\left\|h-h^{\prime}\right\|_{0}<\delta / 2$ and this implies that our chain maps

$$
H^{N \times[0,1]}=g_{13}^{N \times[0,1]}: C(N, h, \gamma) \rightarrow C\left(N, h^{\prime}, \gamma^{\prime}\right)
$$

and $g_{01}^{N \times[0,1]}$ are both isomorphisms. This completes the proof of the Rigidity Theorem 1.19.

Remark 1.23. (a) The proof of the Rigidity Theorem 1.19 (i) can be used to show that for $\mathcal{U}_{h}$ sufficiently small and $H^{\prime}$ a second cobordism satisfying the properties of $H$ the two isomorphisms $H^{N \times[0,1]}$ and $\left(H^{\prime}\right)^{N \times[0,1]}$ differ by a nilpotent chain map.

(b) If $(h, \gamma),\left(h^{\prime}, \gamma^{\prime}\right): N \rightarrow \mathbb{R}$ are Morse-Smale functions with $h^{\prime}$ sufficiently $C^{0}$-close to $h$, the construction of the simple cobordism $H$ in the proof of the Rigidity Theorem 1.19 implies that the resulting chain map

$$
i=H^{N \times[0,1]}: C(N, h, \gamma) \rightarrow C\left(N, h^{\prime}, \gamma^{\prime}\right)
$$

respects the "critical value" filtration of the chain complexes. In particular, if $h^{\prime}=h$ and the critical points of $h$ are alone on their critical levels, then $i$ is a simple isomorphism given in each degree by

$$
i=1+\text { lower triangular matrix }: C_{r}(N, h, \gamma) \rightarrow C_{r}\left(N, h^{\prime}, \gamma^{\prime}\right) .
$$

(c) The Rigidity Theorem 1.19 (iii) shows that a Morse function has simple isomorphic Morse complexes for any two metrics with respect to which it is Morse-Smale. This can also be shown using bifurcation methods as in Latour [12] (p. 21).

1.3. Mayer-Vietoris type formula for the Morse complex. With topological cobordisms one is able to perform two natural, geometric operations. The first associates to a pair formed by a cobordism $\left(M ; N_{0}, N_{1}\right)$ and a separating closed hypersurface $N \subset \operatorname{Int}(M)$ the two cobordisms $\left(M^{\prime} ; N_{0}, N\right)$ and $\left(M^{\prime \prime} ; N, N_{1}\right)$ 
where $M=M^{\prime} \cup_{N} M^{\prime \prime}$. The second is its inverse and it associates to a pair of two cobordisms $\left(M^{\prime} ; N_{0}, N\right)$ and $\left(M^{\prime \prime} ; N, N_{1}\right)$ the new cobordism $\left(M ; N_{0}, N_{1}\right)$ obtained by pasting $M^{\prime}$ to $M^{\prime \prime}$ over $N$. The fact that these two operations are inverses is essential for using Mayer-Vietoris type arguments to deduce properties of a "long" cobordism out of knowledge about the smaller pieces in which it can be divided.

The purpose of this section is to put into place the same two operations for Morse-Smale functions defined on cobordisms and to show a Mayer-Vietoris type theorem for Morse complexes.

Consider the pair formed by a Morse-Smale function $(f, \alpha):\left(M ; N_{0}, N_{1}\right) \rightarrow \mathbb{R}$ and a Morse-Smale function $(g, \beta): N \rightarrow \mathbb{R}$ with $N$ a regular hypersurface of $M$ (we shall assume $N=f^{-1}(0)$ ). In this setting, the first operation will associate to this pair two Morse-Smale functions $h^{\prime}:\left(M^{\prime} ; N_{0}, N\right) \rightarrow \mathbb{R}, h^{\prime \prime}:\left(M^{\prime \prime} ; N, N_{1}\right) \rightarrow \mathbb{R}$ such that $M^{\prime}=f^{-1}(-\infty, 0], M^{\prime \prime}=f^{-1}[0, \infty),\left.h^{\prime}\right|_{N}=k g+c^{\prime},\left.h^{\prime \prime}\right|_{N}=k g+c^{\prime \prime}$ with $k, c^{\prime}, c^{\prime \prime}$ small constants and $h^{\prime}, h^{\prime \prime}$ equal to $f$ away from a neighbourhood of $N$.

Conversely, given two such Morse-Smale functions $h^{\prime}$ and $h^{\prime \prime}$ the second operation produces a Morse-Smale function $h:\left(M ; N_{0}, N_{1}\right) \rightarrow \mathbb{R}$. Obviously, $h$ can not be obtained by simply identifying $h^{\prime}$ and $h^{\prime \prime}$ on $N$ even if $\left.h^{\prime}\right|_{N}=\left.h^{\prime \prime}\right|_{N}=k g+c$ (with $c=c^{\prime}=c^{\prime \prime}$ ) because such an identification does not produce a Morse function. Instead, we consider a linear Morse-Smale cobordism $L: N \times[0,1] \rightarrow \mathbb{R}$ from $k g+c+c^{\prime}=\left.L\right|_{N \times\{0\}}$ to $k g+c^{\prime \prime}=\left.L\right|_{N \times\{1\}}$ with $c>0$ small such that $c+c^{\prime}>c^{\prime \prime}$. We paste $L$ with $h^{\prime}+c$ over $N \times\{0\}$ and then paste the result with $h^{\prime \prime}$ at $N \times\{1\}$ and we thus obtain a Morse- Smale function $h$ as desired.

Contrary to the case of usual cobordisms, if the two operations are applied in succession starting from $f: M \rightarrow \mathbb{R}$, then the resulting function $h$ is quite different from the initial $f$. As $h$ is in fact obtained quite canonically from the pieces $h^{\prime}, h^{\prime \prime}$ in which $f$ has been "split" we shall call such an $h$ a splitting of $f$. This terminology is also justified by the fact that $h$ coincides with $f$ away from a tubular neighbourhood of $N$ and, at the same time, any (negative) gradient flow line of $h$ that passes through a point belonging to $M^{\prime}$ (or to $M^{\prime \prime}$ ) never crosses $N \times\{1 / 2\}$.

We formalize the construction of splittings in Sect. 1.3.1 and remark that it immediate to express the Morse complex of $h$ purely algebraically in terms of the complexes of $h^{\prime}$ and $h^{\prime \prime}$.

It is natural to wonder why in constructing $h^{\prime}, h^{\prime \prime}$ we do not take simply $h^{\prime}=f^{\prime}$, $h^{\prime \prime}=f^{\prime \prime}$ where $f^{\prime}=\left.\right|_{M^{\prime}}, f^{\prime \prime}=\left.f\right|_{M^{\prime \prime}}$. The reason is that the simplest form of a Mayer-Vietoris type formula for Morse complexes should provide the Morse complex of $f$ out of the Morse complexes of the pieces $h^{\prime}$ and $h^{\prime \prime}$. However, the Morse complexes of $f^{\prime}$ and $f^{\prime \prime}$ are inappropriate for this task because they do not encode the flow lines of $-\nabla f$ that cross from $M^{\prime \prime}$ into $M^{\prime}$. On the other hand, the gluing Theorem 1.29 in Sect. 1.3.2 shows that if $g$ satisfies a certain, generic, technical condition, then the Morse complexes of $h^{\prime}$ and $h^{\prime \prime}$ can be "glued" together in a canonical way to give the Morse complex of $f$. The isomorphism type of the Morse complex of $f$ can be recovered out of any splitting of $f$ even if the technical condition is not satisfied. 
1.3.1. Splittings. In this section we consider a Morse-Smale function

$$
(f, \alpha):\left(M ; N_{0}, N_{1}\right) \rightarrow \mathbb{R}
$$

with $f\left(x_{0}\right)<0$ for all $x_{0} \in N_{0}, f\left(x_{1}\right)>0$ for all $x_{1} \in N_{1}$, and $0 \in \mathbb{R}$ a regular value of $f$. We write

$$
N=f^{-1}(0) \subset M
$$

The restrictions

$$
\begin{aligned}
& \left(f^{\prime}, \alpha^{\prime}\right)=(f, \alpha) \mid:\left(M^{\prime} ; N_{0}, N\right)=f^{-1}(-\infty, 0] \rightarrow \mathbb{R}, \\
& \left(f^{\prime \prime}, \alpha^{\prime \prime}\right)=(f, \alpha) \mid:\left(M^{\prime \prime} ; N, N_{1}\right)=f^{-1}[0, \infty) \rightarrow \mathbb{R}
\end{aligned}
$$

are Morse-Smale functions with

$$
\begin{aligned}
& (f, \alpha)=\left(f^{\prime}, \alpha^{\prime}\right) \cup\left(f^{\prime \prime}, \alpha^{\prime \prime}\right): \\
& \left(M ; N_{0}, N_{1}\right)=\left(M^{\prime} ; N_{0}, N\right) \cup_{N}\left(M^{\prime \prime} ; N, N_{1}\right) \rightarrow \mathbb{R} .
\end{aligned}
$$

Definition 1.24. The attaching chain map

$$
\phi: C\left(M^{\prime \prime}, f^{\prime \prime}, \alpha^{\prime \prime}\right)_{*+1} \rightarrow C\left(M^{\prime}, f^{\prime}, \alpha^{\prime}\right)
$$

is the chain map given by

$$
\phi: C\left(M^{\prime \prime}, f^{\prime \prime}, \alpha^{\prime \prime}\right)_{i+1} \rightarrow C\left(M^{\prime}, f^{\prime}, \alpha^{\prime}\right)_{i} ; x \mapsto \sum_{y \in \operatorname{Crit}_{i}\left(f^{\prime}\right)} n^{f, \alpha}(x, y) y .
$$

Proposition 1.25. The Morse complexes fit into a short exact sequence

$$
0 \rightarrow C\left(M^{\prime}, f^{\prime}, \alpha^{\prime}\right) \rightarrow C(M, f, \alpha) \rightarrow C\left(M^{\prime \prime}, f^{\prime \prime}, \alpha^{\prime \prime}\right) \rightarrow 0
$$

with

$$
C(M, f, \alpha)=C(\phi)
$$

the algebraic mapping cone of the attaching chain map

$$
\phi: C\left(M^{\prime \prime}, f^{\prime \prime}, \alpha^{\prime \prime}\right)_{*+1} \rightarrow C\left(M^{\prime}, f^{\prime}, \alpha^{\prime}\right) .
$$

Proof. Immediate from the definitions.

The key technical construction of this section is contained in the following definition. To fix ideas we will proceed from here under the following assumption: there exists a neighbourhood $N \times[-\delta, \delta] \subset M$ of $N$ such that on this neighbourhood $f$ has the form $f(x, t)=t$ and the metric $\alpha$ has the form $d t^{2}+\beta, \beta=\left.\alpha\right|_{N}$. The existence of such a parametrization of $f$ around $N$ is clear from the fact that $N$ is a regular level hypersurface of $f$. Moreover, because $\partial / \partial t$ is orthogonal to $N \times\{t\}$ the metric $\alpha$ has the form $u(x, t) d t^{2}+v(x, t) \beta$ (with $u, v$ smooth positive functions) on our neighbourhood. This means that by replacing $\alpha$ in this neighbourhood with the product metric we do not perturb the flow lines of $f$. Therefore, our assumption is in no way restrictive. 
Definition 1.26. An $(\epsilon, \tau)$-splitting of a Morse-Smale function

$$
(f, \alpha):\left(M ; N_{0}, N_{1}\right) \rightarrow \mathbb{R}
$$

along a Morse-Smale function $(g, \beta): N=f^{-1}(0) \rightarrow \mathbb{R}(\beta=\alpha \mid)$ is a MorseSmale function $(h, \alpha): M \rightarrow \mathbb{R}$ such that for $\left(h^{\prime}, \alpha^{\prime}\right)=\left.(h, \alpha)\right|_{M^{\prime}},\left(h^{\prime \prime}, \alpha^{\prime \prime}\right)=$ $\left.(h, \alpha)\right|_{M^{\prime \prime}}$ we have

(i) $\left.\quad h^{\prime}\right|_{N}=\left.h^{\prime \prime}\right|_{N}=\tau g: N \rightarrow \mathbb{R}$ with $\tau \in \mathbb{R}^{+}$.

(ii) $(h, \alpha)=(f, \alpha)$ except in a small tubular neighbourhood $W=N \times[-\epsilon, \epsilon] \subset$ $M, \epsilon \in \mathbb{R}^{+}$, of $N=N \times\{0\} \subset M$.

(iii) With respect to this parametrization $f(x, t)=t$ for all $(x, t) \in N \times[-\epsilon, \epsilon]$ and

$$
\begin{aligned}
& \operatorname{Crit}_{i}(h)=\operatorname{Crit}_{i}(f) \cup\left(\operatorname{Crit}_{i-1}(g) \times\{-\epsilon / 2\}\right) \cup\left(\operatorname{Crit}_{i}(g) \times\{\epsilon / 2\}\right), \\
& \operatorname{Crit}_{i}\left(h^{\prime}\right)=\operatorname{Crit}_{i}\left(f^{\prime}\right) \cup\left(\operatorname{Crit}_{i-1}(g) \times\{-\epsilon / 2\}\right), \\
& \operatorname{Crit}_{i}\left(h^{\prime \prime}\right)=\operatorname{Crit}_{i}\left(f^{\prime \prime}\right) \cup\left(\operatorname{Crit}_{i}(g) \times\{\epsilon / 2\}\right) .
\end{aligned}
$$

(iv) The restrictions of $(h, \alpha)$ to the submanifolds

$$
\begin{aligned}
& W_{\epsilon}=N \times[-\epsilon / 2, \epsilon / 2] \subset W, \\
& M_{\epsilon}^{\prime}=M^{\prime} \backslash(N \times(-\epsilon / 2,0]) \subset M^{\prime}, \\
& M_{\epsilon}^{\prime \prime}=M^{\prime \prime} \backslash(N \times[0, \epsilon / 2)) \subset M^{\prime \prime}
\end{aligned}
$$

(which are copies of $W, M^{\prime}, M^{\prime \prime}$ respectively) are Morse-Smale cobordisms

$$
\begin{aligned}
& \left(g_{\epsilon}, \beta_{\epsilon}\right)=(h, \alpha) \mid:\left(W_{\epsilon} ; N \times\{\epsilon / 2\}, N \times\{-\epsilon / 2\}\right) \rightarrow \mathbb{R}, \\
& \left(h_{\epsilon}^{\prime}, \alpha_{\epsilon}^{\prime}\right)=\left(h^{\prime}, \alpha^{\prime}\right) \mid:\left(M_{\epsilon}^{\prime} ; N_{0}, N \times\{-\epsilon / 2\}\right) \rightarrow \mathbb{R}, \\
& \left(h_{\epsilon}^{\prime \prime}, \alpha_{\epsilon}^{\prime \prime}\right)=\left(h^{\prime \prime}, \alpha^{\prime \prime}\right) \mid:\left(M_{\epsilon}^{\prime \prime} ; N \times\{\epsilon / 2\}, N_{1}\right) \rightarrow \mathbb{R} .
\end{aligned}
$$

(v) The cobordism $\left(g_{\epsilon}, \beta_{\epsilon}\right)$ is linear.

The key properties of a splitting are collected next.

Proposition 1.27. Let $(f, \alpha):\left(M ; N_{0}, N_{1}\right) \rightarrow \mathbb{R}$ be a Morse-Smale function, with $0 \in \mathbb{R}$ a regular value.

(i) For every Morse-Smale function $(g, \beta): N=f^{-1}(0) \rightarrow \mathbb{R}$ with $\beta=\left.\alpha\right|_{N}$ and every $\delta>0$ there exists an $(\epsilon, \tau)$-splitting $(h, \alpha)$ of $(f, \alpha)$ along $(g, \beta)$ with $\|h-f\|_{0} \leq \delta$.

(ii) The Morse complexes of a splitting of $(f, \alpha)$ along $(g, \beta)$

$$
\begin{aligned}
& (h, \alpha)=\left(h_{\epsilon}^{\prime}, \alpha_{\epsilon}^{\prime}\right) \cup\left(g_{\epsilon}, \beta_{\epsilon}\right) \cup\left(h_{\epsilon}^{\prime \prime}, \alpha_{\epsilon}^{\prime \prime}\right): \\
& \begin{aligned}
&\left(M ; N_{0}, N_{1}\right)=\left(M_{\epsilon}^{\prime} ; N_{0}, N \times\{-\epsilon / 2\}\right) \cup\left(W_{\epsilon} ; N \times\{-\epsilon / 2\}, N \times\{\epsilon / 2\}\right) \\
& \cup\left(M_{\epsilon}^{\prime \prime} ; N \times\{\epsilon / 2\}, N_{1}\right) \rightarrow \mathbb{R},
\end{aligned}
\end{aligned}
$$




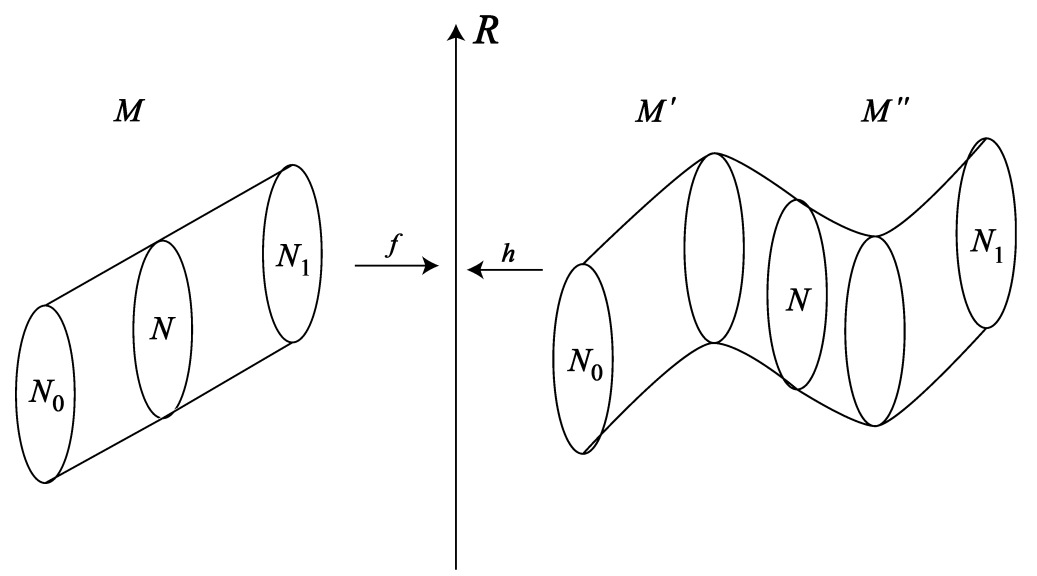

$f$ and its splitting $h$

are given by

$$
\begin{aligned}
& C(M, h, \alpha)=C(\theta), \\
& C\left(M_{\epsilon}^{\prime}, h_{\epsilon}^{\prime}, \alpha_{\epsilon}^{\prime}\right)=C\left(M^{\prime}, h^{\prime}, \alpha^{\prime}\right)=C\left(\theta^{\prime}\right), \\
& C\left(M_{\epsilon}^{\prime \prime}, h_{\epsilon}^{\prime \prime}, \alpha_{\epsilon}^{\prime \prime}\right)=C\left(M^{\prime \prime}, h^{\prime \prime}, \alpha^{\prime \prime}\right)=C\left(\theta^{\prime \prime}\right) \\
& C\left(W_{\epsilon}, g_{\epsilon}, \beta_{\epsilon}\right)=C(1: C(N, g, \beta) \rightarrow C(N, g, \beta))
\end{aligned}
$$

with

$$
\begin{aligned}
& \theta: C\left(M^{\prime}, h^{\prime}, \alpha^{\prime}\right)_{*+1} \rightarrow C\left(M^{\prime \prime}, h^{\prime \prime}, \alpha^{\prime \prime}\right), \\
& \theta^{\prime}: C(N, g, \beta) \rightarrow C\left(M^{\prime}, f^{\prime}, \alpha^{\prime}\right), \\
& \theta^{\prime \prime}: C\left(M^{\prime \prime}, f^{\prime \prime}, \alpha^{\prime \prime}\right)_{*+1} \rightarrow C(N, g, \beta)
\end{aligned}
$$

the chain maps (up to sign) defined by

$$
\begin{array}{r}
\theta: C\left(M^{\prime}, h^{\prime}, \alpha^{\prime}\right)_{i+1}=C\left(M^{\prime}, f^{\prime}, \alpha^{\prime}\right)_{i+1} \oplus C(N, g, \beta)_{i} \\
\rightarrow C\left(M^{\prime \prime}, h^{\prime \prime}, \alpha^{\prime \prime}\right)_{i}=C(N, g, \beta)_{i} \oplus C\left(M^{\prime \prime}, f^{\prime \prime}, \alpha^{\prime \prime}\right)_{i} ; \\
\quad(x, z \times\{-\epsilon / 2\}) \mapsto(z \times\{\epsilon / 2\}, 0) \quad\left(x \in \mathrm{Crit}_{i+1}\left(f^{\prime}\right), z \in \mathrm{Crit}_{i}(g)\right), \\
\theta^{\prime}: C(N, g, \beta)_{i} \rightarrow C\left(M^{\prime}, f^{\prime}, \alpha^{\prime}\right)_{i} ; z \mapsto \sum_{y \in \mathrm{Crit}_{i}\left(f^{\prime}\right)} n^{h^{\prime}, \alpha^{\prime}}(z \times\{-\epsilon / 2\}, y) y, \\
\theta^{\prime \prime}: C\left(M^{\prime \prime}, f^{\prime \prime}, \alpha^{\prime \prime}\right)_{i+1} \rightarrow C(N, g, \beta)_{i} ; x \mapsto \sum_{z \in \operatorname{Crit}_{i}(g)} n^{h^{\prime \prime}, \alpha^{\prime \prime}}(x, z \times\{\epsilon / 2\}) z .
\end{array}
$$

In particular, there are defined exact sequences

$$
\begin{aligned}
& 0 \rightarrow C\left(M^{\prime \prime}, h^{\prime \prime}, \alpha^{\prime \prime}\right) \rightarrow C(M, h, \alpha) \rightarrow C\left(M^{\prime}, h^{\prime}, \alpha^{\prime}\right) \rightarrow 0, \\
& 0 \rightarrow C\left(M^{\prime}, f^{\prime}, \alpha^{\prime}\right) \rightarrow C\left(M^{\prime}, h^{\prime}, \alpha^{\prime}\right) \rightarrow C(N, g, \beta)_{*-1} \rightarrow 0, \\
& 0 \rightarrow C(N, g, \beta) \rightarrow C\left(M^{\prime \prime}, h^{\prime \prime}, \alpha^{\prime \prime}\right) \rightarrow C\left(M^{\prime \prime}, f^{\prime \prime}, \alpha^{\prime \prime}\right) \rightarrow 0 .
\end{aligned}
$$


(iii) Let $p_{h}: C\left(W_{\epsilon}, g_{\epsilon}, \beta_{\epsilon}\right) \rightarrow C(M, h, \alpha)$ be the chain map defined by the split injections

$$
\begin{aligned}
& p_{h}: C\left(W_{\epsilon}, g_{\epsilon}, \beta_{\epsilon}\right)_{i}=C(N, g, \beta)_{i-1} \oplus C(N, g, \beta)_{i} \rightarrow \\
& C(M, h, \alpha)_{i} \\
& =C\left(M^{\prime}, f^{\prime}, \alpha^{\prime}\right)_{i} \oplus C(N, g, \beta)_{i-1} \oplus C(N, g, \beta)_{i} \oplus C\left(M^{\prime \prime}, f^{\prime \prime}, \alpha^{\prime \prime}\right)_{i} ; \\
& (x, y) \mapsto\left(\theta^{\prime}(y), x, y, 0\right) .
\end{aligned}
$$

The algebraic mapping cone of $p_{h}$ is the Morse complex of a splitting $(h, \alpha)$ : $M \rightarrow \mathbb{R}$

$$
C\left(p_{h}\right)=C(M, h, \alpha) .
$$

The cokernel of $p_{h}$ is the algebraic mapping cone of $\theta^{\prime} \theta^{\prime \prime}: C\left(M^{\prime \prime}, f^{\prime \prime}, \alpha^{\prime \prime}\right)_{*+1} \rightarrow$ $C\left(M^{\prime}, f^{\prime}, \alpha^{\prime}\right)$

$$
0 \rightarrow C\left(W_{\epsilon}, g_{\epsilon}, \beta_{\epsilon}\right) \stackrel{p_{h}}{\rightarrow} C(M, h, \alpha) \stackrel{j_{h}}{\rightarrow} C\left(\theta^{\prime} \theta^{\prime \prime}\right) \rightarrow 0,
$$

with $j_{h}$ the natural projection.

Proof. (i) This follows easily from the constructions in Sect. 1.1.3. We first choose $a>0$ such that $[-a, a]$ contains only regular values of $f$, with

$$
f \mid: W=f^{-1}([-a, a])=N \times[-a, a] \rightarrow \mathbb{R} ;(x, t) \mapsto t
$$

and the assumption that $\alpha$ is the product metric here. After possibly multiplying $g$ with a sufficiently small constant we may assume that the image of $g$ belongs to $[-b, b]$ with $b \in(0,1)$ such that $b \ll a$. Let

$$
\begin{aligned}
& N_{\delta}=N \times\{\delta\} \subset W=N \times[-a, a], \\
& W(u, v)=N \times[u, v] \subset W .
\end{aligned}
$$

By using Lemmas 1.13, 1.14 for each $0<\epsilon<b$ we construct a Morse-Smale function $f_{\epsilon}: M \rightarrow \mathbb{R}$ of $f$ along $g$ such that: $f_{\epsilon}$ agrees with $f$ outside $W(-\epsilon, \epsilon)$; the gradient of $f_{\epsilon}$ is everywhere tangent to $N_{\epsilon / 2}$ and $N_{-\epsilon / 2}$,

$$
\left.f_{\epsilon}\right|_{N_{\epsilon / 2}}=\epsilon(g+k),\left.f_{\epsilon}\right|_{N_{-\epsilon / 2}}=\epsilon\left(g+k^{\prime}\right)
$$

with $k^{\prime}>k$ small; if $x \in \operatorname{Crit}_{f_{\epsilon}} \cap N_{\epsilon / 2}$ then $\operatorname{ind}_{f_{\epsilon}}(x)=\operatorname{ind}_{g}(x)$ and if $x \in$ Crit $_{f_{\epsilon}} \cap N_{-\epsilon / 2}$, then $\operatorname{ind}_{f_{\epsilon}}(x)=\operatorname{ind}_{g}(x)+1$; on the cobordism $(W(-\epsilon / 2, \epsilon / 2)$; $\left.N_{-\epsilon / 2}, N_{\epsilon / 2}\right)$ the function $f_{\epsilon}$ restricts to a linear cobordism. The construction of the function $f_{\epsilon}$ is done by applying Lemma 1.13 to the two cobordisms $W(-\epsilon,-\epsilon / 2)$ and $W(\epsilon / 2, \epsilon)$ and Lemma 1.14 to the cobordism $W(-\epsilon / 2, \epsilon / 2)$. In particular, $f_{\epsilon}$ is a linear combination of the form $u(t)(-\epsilon)+(1-u(t)) \epsilon\left(g+k^{\prime}\right)$ inside $N \times$ $[-\epsilon,-\epsilon / 2]$ with $u:[-\epsilon,-\epsilon / 2] \rightarrow \mathbb{R}$ an appropriate function with $u(-\epsilon)=1$ and $u(-\epsilon / 2)=0=u^{\prime}(-\epsilon / 2), u^{\prime \prime}(-\epsilon / 2)>0$ (see 1.13); a similar linear combination is valid inside $N \times[\epsilon / 2, \epsilon /]$. To understand the pasting between the cobordism $W(-\epsilon / 2, \epsilon / 2)$ and, for example, $W(-\epsilon,-\epsilon / 2)$ notice that on $W(-\epsilon / 2, \epsilon / 2)$ we 
have a cobordism from $\left(\epsilon\left(g+k^{\prime}\right), N_{-\epsilon / 2}\right)$ to $\left(\epsilon(g+k), N_{\epsilon / 2}\right)$. In other words, on both sides of $N_{-\epsilon / 2}$ we have cobordisms having $N_{-\epsilon / 2}$ as "high end". This allows the pasting to occur by simply using in the formula above $u$ defined on a larger interval, say $u:[-\epsilon, 0] \rightarrow \mathbb{R}$, and using the same formula to define $f_{\epsilon}$ on both sides of $N_{-\epsilon / 2}$. For a generic choice of $g$ the function $f_{\epsilon}$ is already Morse-Smale with respect to the metric $\alpha$. In general, to obtain a function $h$ such that $(h, \alpha)$ is Morse-Smale we might need to still slightly perturb $f_{\epsilon}$ inside $N \times([-\epsilon,-3 \epsilon / 4] \cup[3 \epsilon / 4, \epsilon])$. We may assume that the construction has been made such that $\left\|f-f_{\epsilon}\right\|_{0} \leq 5 \epsilon$ and by adjusting the various relevant constants we see that $(h, \alpha)$ is a splitting of $f$ along $g$ with the required properties.

(ii) + (iii) Immediate from the definition and construction of a splitting.

1.3.2. Assembly of the Morse complex. Given the algebraic data extracted from a splitting of a Morse-Smale function $(f, \alpha):\left(M ; N_{0}, N_{1}\right) \rightarrow \mathbb{R}$ we now describe how to reconstruct the Morse complex of $f$ from the Morse complexes of $h^{\prime}$ and $h^{\prime \prime}$. One expects the composite chain map

$$
\theta^{\prime} \theta^{\prime \prime}: C\left(M^{\prime \prime}, f^{\prime \prime}, \alpha^{\prime \prime}\right)_{*+1} \rightarrow C\left(M^{\prime}, f^{\prime}, \alpha^{\prime}\right)
$$

to be a good approximation of the attaching chain map $\phi$.

Definition 1.28. A Morse-Smale function $(g, \beta): N=f^{-1}(0) \rightarrow \mathbb{R}$ is adapted to splitting $(f, \alpha):\left(M ; N_{0}, N_{1}\right) \rightarrow \mathbb{R}$ at $N \subset M$ if

(i) $(g, \beta): N \rightarrow \mathbb{R}$ is Morse-Smale, with $\beta=\left.\alpha\right|_{N}$.

(ii) For any $p \in \mathrm{Crit}_{i}(f)$

$$
W_{f}^{u}(p) \bigcap N \subset \bigcup_{z \in \mathrm{Crit}_{\leq i-1}(g)} W_{g}^{u}(z) .
$$

(iii) For any $q \in \mathrm{Crit}_{*}(f)$ and any $z \in \mathrm{Crit}_{*}(g)$ the intersections $W_{f}^{u}(q) \bigcap W_{g}^{s}(z)$ and $W_{g}^{u}(z) \cap W_{f}^{s}(q)$ are transversal.

Gluing Theorem 1.29. Let $(f, \alpha):\left(M ; N_{0}, N_{1}\right) \rightarrow \mathbb{R}$ be a Morse-Smale function, with $N=f^{-1}(0)$ a regular hypersurface.

(i) Let $(g, \beta): N \rightarrow \mathbb{R}$ be a Morse-Smale function. For any $(\epsilon, \tau)$-splitting $(h, \alpha)$ of $f$ along $g$ with $\epsilon, \tau$ sufficiently small there exists a chain homotopy $\psi: \phi \simeq \theta^{\prime} \theta^{\prime \prime}$, defining a simple isomorphism

$$
\left(\begin{array}{ll}
1 & \psi \\
0 & 1
\end{array}\right): C(M, f, \alpha) \stackrel{\cong}{\rightarrow} \operatorname{coker}\left(p_{h}\right) .
$$

(ii) There exist Morse-Smale functions $(g, \beta)$ adapted to splitting $(f, \alpha)$.

(iii) If $(g, \beta)$ is adapted to splitting $(f, \alpha)$ at $N$, then there exist splittings $h$ of $f$ along $g$ with $\psi=0$. Equivalently, we have the equality $\phi=\theta^{\prime} \theta^{\prime \prime}$ or, explicitly:

$$
n^{f, \alpha}(x, y)=\sum_{z \in \mathrm{Crit}_{i-1}(g)} n^{h^{\prime \prime}, \alpha^{\prime \prime}}(x, z \times\{\epsilon / 2\}) n^{h^{\prime}, \alpha^{\prime}}(z \times\{-\epsilon / 2\}, y)
$$

for each pair $x \in \mathrm{Crit}_{i}\left(f^{\prime \prime}\right), y \in \mathrm{Crit}_{i-1}\left(f^{\prime}\right)$. 
Remark 1.30. To our knowledge, a gluing formula as the one above has not yet appeared in the literature. However, a closely related result is that of Laudenbach [13] and has been extended to $S^{1}$-valued functions by Hutchings [10]. To see the relation with these results notice that, in the situation of Proposition 1.27, the based f.g. free $\mathbb{Z}[\pi]$-module chain complex

$$
\operatorname{coker}\left(p_{h}\right)=C\left(\theta^{\prime} \theta^{\prime \prime}\right)
$$

is the Morse complex $C(M, \widehat{f}, \alpha)$ of a Morse-Smale function $(\widehat{f}, \alpha): M \rightarrow \mathbb{R}$ obtained from $(h, \alpha)$ by cancelling the pairs of critical points added in the construction of $h$ from $f$, with

$$
\mathrm{Crit}_{*}(\widehat{f})=\mathrm{Crit}_{*}(f)
$$

Indeed, the results of Laudenbach describe the modifications occurring in the Morse complex after the birth (or death) of two mutually annihilating critical points of successive indexes. By applying iteratively his result when cancelling all such pairs of critical points of $h$ that belong to $W_{\epsilon}$ one obtains a function $\widehat{f}$ whose Morse complex does equal $C\left(\theta^{\prime} \theta^{\prime \prime}\right)$. Moreover, this function may be assumed to be $C^{0}$-close to $f$. Therefore, this argument, together with the rigidity theorem, is sufficient to prove the point (i) of the theorem. On the other hand, this is not enough to show the point (iii). The reason is that "cancelling of critical points" is a non-unique operation and there is no way to insure in general that the function $\widehat{f}$ obtained at the end of this process is $C^{2}$-close to $f$ and has therefore a Morse complex identical to that of $f$. Because of this we shall prove the whole theorem without the use of bifurcation arguments (as we shall see, the point (i) is in fact immediate by using Morse cobordisms).

Other constructions related to the gluing formula appear in Pajitnov [16]. The role of our formula is played there by an analysis of the intersections of stable and unstable manifolds of $f$ with the stable and unstable manifolds of $g$.

Proof. (i) This point is quite immediate. Assume $(h, \alpha)$ is an $(\epsilon, \tau)$-splitting of $f$ along $g$ as in the statement. By the constructions in Sect. 1.1.3 there is a Morse cobordism $H$ between $(h, \alpha)$ and $(f+c, \alpha)$. If $\epsilon$ is small enough, then $h$ is sufficiently $C^{0}$ close to $f$ such that

$$
\begin{aligned}
& \min \left\{f\left(\mathrm{Crit}_{*}\left(f^{\prime \prime}\right)\right)\right\} \geq \max \left\{h\left(\mathrm{Crit}_{*}(h)-\mathrm{Crit}_{*}(f)\right)\right\}, \\
& \min \left\{h\left(\mathrm{Crit}_{*}(h)-\mathrm{Crit}_{*}(f)\right)\right\}>c+\max \left\{f\left(\mathrm{Crit}_{*}\left(f^{\prime}\right)\right)\right\}
\end{aligned}
$$

and we may construct $H$ such that it restricts to linear cobordisms with a product metric on $M^{\prime} \backslash(N \times(-\epsilon, \epsilon))$ and on $M^{\prime \prime} \backslash(N \times(-\epsilon, \epsilon))$ (we use the notations in Proposition 1.27). Denote by $G: C(M, f, \alpha) \rightarrow C(M, h, \alpha)$ the chain map induced by this cobordism. Due to the linearity of $H$ on the complement of $N \times(-\epsilon, \epsilon)$ the composite

$$
j^{\prime}=j_{h} \circ G: C(M, f, \alpha) \rightarrow \operatorname{coker}\left(p_{h}\right)
$$


is a simple isomorphism of the form

$$
\begin{aligned}
j^{\prime}=\left(\begin{array}{ll}
1 & \psi \\
0 & 1
\end{array}\right): C(M, f, \alpha)_{i} & =C\left(M^{\prime}, f^{\prime}, \alpha^{\prime}\right)_{i} \oplus C\left(M^{\prime \prime}, f^{\prime \prime}, \alpha^{\prime \prime}\right)_{i} \\
& \rightarrow \operatorname{coker}\left(p_{h}\right)_{i}=C\left(M^{\prime}, f^{\prime}, \alpha^{\prime}\right)_{i} \oplus C\left(M^{\prime \prime}, f^{\prime \prime}, \alpha^{\prime \prime}\right)_{i}
\end{aligned}
$$

for a chain homotopy

$$
\psi: \phi \simeq \theta^{\prime} \theta^{\prime \prime}: C\left(M^{\prime \prime}, f^{\prime \prime}, \alpha^{\prime \prime}\right)_{*+1} \rightarrow C\left(M^{\prime}, f^{\prime}, \alpha^{\prime}\right) .
$$

(ii) Clearly, a Morse-Smale function $(g, \beta): N \rightarrow \mathbb{R}$ is not in general adapted to splitting $(f, \alpha): M \rightarrow \mathbb{R}$. We obtain an adapted $(g, \beta)$ by starting with an arbitrary $\left(g^{\prime}, \beta\right): N \rightarrow \mathbb{R}$ and adding pairs of mutually cancelling critical points such that the unstable manifolds of $g$ give a $C W$ decomposition of $N$ which is sufficiently fine to ensure that Definition 1.28 (ii) is verified. In particular, this means that $W_{f}^{u}(R) \bigcap W_{g^{\prime}}^{u}\left(R^{\prime}\right)=\emptyset$ whenever $\operatorname{ind}_{g^{\prime}}\left(R^{\prime}\right) \geq \operatorname{ind}_{f}(R)$. After further subdivision we may also assume that for any pair $R^{\prime} \in \mathrm{Crit}_{*}\left(g^{\prime}\right), R \in \mathrm{Crit}_{*}(f)$ we either have $W_{f}^{u}(R) \cap W_{g^{\prime}}^{u}\left(R^{\prime}\right)=\emptyset$, or $W_{g^{\prime}}^{u}\left(R^{\prime}\right) \subset W_{f}^{u}(R)$. We then modify $g^{\prime}$ such as to perturb the stable manifolds $W_{g^{\prime}}^{s}\left(R^{\prime \prime}\right)$ to render them transverse to $W_{f}^{u}(R)$ for all pairs $R^{\prime \prime} \in \mathrm{Crit}_{*}\left(g^{\prime}\right), R \in \mathrm{Crit}_{*}(f)$. This modification is performed successively for $R^{\prime \prime}$ of increasing indexes and it may be achieved without modifying those unstable manifolds $W_{g^{\prime}}^{u}\left(R^{\prime}\right)$ which intersect some unstable manifold of $f$. This happens because if $W_{g^{\prime}}^{u}\left(R^{\prime}\right)$ intersects $W_{f}^{u}(R)$, then $W_{g^{\prime}}^{u}\left(R^{\prime}\right) \subset W_{f}^{u}(R)$ and as $g^{\prime}$ is Morse-Smale we also have that $W_{g^{\prime}}^{s}\left(R^{\prime \prime}\right)$ is transversal to $W_{g^{\prime}}^{u}\left(R^{\prime}\right)$ (for all $R^{\prime}, R^{\prime \prime} \in$ Crit $\left._{*}\left(g^{\prime}\right)\right)$. This means that if $W_{g^{\prime}}^{s}\left(R^{\prime \prime}\right)$ intersects $W_{f}^{u}(R)$ in a point which belongs also to $W_{g^{\prime}}^{u}\left(R^{\prime}\right)$, then $W_{g^{\prime}}^{s}\left(R^{\prime \prime}\right)$ is already transversal to $W_{f}^{u}(R)$ at this point. We also need to modify our function $g^{\prime}$ such as to obtain one which also has the property that the second type of intersections in Definition 1.28 (iii) are transversal. We perturb successively each unstable manifold $W_{g^{\prime}}^{u}\left(R^{\prime}\right), R^{\prime} \in \mathrm{Crit}_{*}\left(g^{\prime}\right)$ such as to make $W_{g^{\prime}}^{u}\left(R^{\prime}\right)$ transversal to the stable manifolds of $f$. Obviously, we need to insure that condition (ii) in Definition 1.28 is preserved and therefore the critical points $R^{\prime}$ such that $W_{g^{\prime}}^{u}\left(R^{\prime}\right)$ intersects some $W_{f}^{u}(R)$ need to be discussed separately. In this case we have $W_{g^{\prime}}^{u}\left(R^{\prime}\right) \subset W_{f}^{u}(R)$. Suppose that ind $f(R)=\operatorname{ind}_{g^{\prime}}\left(R^{\prime}\right)+1$, then as $W_{f}^{u}(R)$ is transverse to all stable manifolds $W_{f}^{s}(Q)$ (by the Morse- Smale condition) it also follows that $W_{g^{\prime}}^{u}\left(R^{\prime}\right)$ is transverse to these stable manifolds and no modification of $W_{g^{\prime}}^{u}\left(R^{\prime}\right)$ is needed. In case $\operatorname{ind}_{g^{\prime}}\left(R^{\prime}\right)<\operatorname{ind}_{f}(R)+1$ it follows from condition (ii) in Definition 1.28 that $W_{g^{\prime}}^{u}\left(R^{\prime}\right) \subset W_{f}^{u}(R) \cap N \subset \bigcup_{\text {ind }_{g^{\prime}}(z)=\operatorname{ind}_{f}(P)-1} \overline{W_{g^{\prime}}^{u}(z)}=T$. We then perturb $W_{g^{\prime}}^{u}\left(R^{\prime}\right)$ inside $W_{f}^{u}(R)$ such as to obtain the needed transversality. This will also perturb the higher dimensional $W_{g^{\prime}}^{u}(z)$ 's that appear in the union $T$ above but can be made such that $T$ does not diminish and thus (ii) of Definition 1.28 continues to be satisfied.

It is useful to note that one can obtain in this way an adapted $g$ that is arbitrarily close in $C^{0}$ norm to the function $g^{\prime}$ that was given initially.

(iii) We now assume that $(g, \beta)$ is adapted to splitting $(f, \alpha)$. Recall the functions $f_{\epsilon}$ constructed in the proof of Proposition 1.27. We shall work below with functions 
of this form and with the notations of that proof. In particular,

$$
W=f^{-1}([-a, a]), W(u, v)=N \times[u, v]=f^{-1}([u, v]), N=f^{-1}(0) .
$$

It is immediate to see that because of the transversality properties of the adapted $g$, the function $f_{\epsilon}$ is Morse-Smale with respect to $\alpha$. Therefore $\left(f_{\epsilon}, \alpha\right)$ is itself a splitting and we will show below that any such splitting with $(\epsilon, \tau)$ sufficiently small verifies the conclusion.

By inspecting the construction in 1.27, we see that, in each point of $W(-\epsilon, \epsilon)$, the tangent vector $\nabla f_{\epsilon}$ projected onto $N$ has the same direction as $\nabla g$. We intend to compare the Morse complex of $f$ with that of $f_{\epsilon}$ using a special Morse cobordism between $f_{\epsilon}$ and $f+c$. For this we start with a linear cobordism $F: M \times[0,1] \rightarrow \mathbb{R}$ of Morse-Smale functions between $f$ and $f+c$ with $c>0$ fixed. We assume $F_{0}=f$ and $F_{1}=f+c$. We denote by $\tau \in[0,1]$ the deformation parameter of $F$. We may also assume that in a neighbourhood $M \times[0,1 / 3]$ of $M \times\{0\}$ the function $F$ has the form $F=f+\tau^{2}$. We shall identify below $M$ and $M \times\{0\}$ (for example we write $\left.F\right|_{M \times\{0\}}=f$ etc).

We want to show that we can modify $F$ only inside $M^{\epsilon}=W(-\epsilon, \epsilon) \times$ $\left[0,(5 \epsilon)^{1 / 3}\right]$ such that the resulting function $F^{\epsilon}$ will be a Morse-Smale cobordism of $f_{\epsilon}$ and $f+c$. By a similar method to to that used in 1.13 we can define $F^{\epsilon}$ inside $M^{\epsilon}$ by

$$
\left.F^{\epsilon}\right|_{M^{\epsilon}}=\tau^{2}+v(\tau) f+(1-v(\tau)) f_{\epsilon}
$$

where

$$
\begin{aligned}
& v:[0,1] \rightarrow[0,1] \text { is } C^{\infty} \\
& v \text { is constant equal to } 1 \text { if } \tau>(5 \epsilon)^{1 / 3} \\
& v(0)=v^{\prime}(0)=v^{\prime \prime}(0)=0 \\
& \left|v^{\prime}(\tau) 5 \epsilon\right|<2 \tau \text { for all } \tau .
\end{aligned}
$$

(Such a function exists, it can be modelled on $\tau^{3} / 5 \epsilon$ ) and $F^{\epsilon}=F$ outside of $M^{\epsilon}$. The function $F^{\epsilon}$ is smooth because $f^{\epsilon}=f$ outside of $W(-\epsilon, \epsilon)$ and it is clear that it is Morse. Note for each point of $M^{\epsilon}$ the gradient of $F^{\epsilon}$ with respect to the product metric $\alpha+d \tau^{2}$ decomposes as an orthogonal sum of a component tangent to $N_{0}$ which is a multiple of the gradient of $g$ and two other components, one in the direction of $\partial / \partial t$, and the other into that of $\partial / \partial \tau$. We can change slightly the metric $\alpha+d \tau^{2}$ inside a set $M \times\left[l, l^{\prime}\right]$ with $(5 \epsilon)^{1 / 3}<l<l^{\prime}<1$ thus getting a metric $\alpha_{\epsilon}$ sufficiently close to $\alpha$ such that with respect to this new metric $F$ remains Morse-Smale and its complex is not modified and $F^{\epsilon}$ becomes also Morse-Smale. In fact, the set of metrics $\alpha^{*}$ such that $F$ remains Morse-Smale with respect to $\alpha^{*}$ and has the same Morse complex as with respect to $\alpha$ is dense and open in some neighborhood of $\alpha$. Therefore, if we now consider the sequence of functions $F^{1 / n}, n \in \mathbf{N}^{*}, n \geq n_{0} \gg 1$, we see that there is a metric $\alpha^{\prime}$ on $M \times[0,1]$ such that $\alpha^{\prime}$ equals $\alpha+d \tau^{2}$ outside $M \times[1 / 3,2 / 3]$, for all $n, F^{1 / n}$ is Morse-Smale with respect to $\alpha^{\prime}$ and $F$ is also Morse-Smale with respect to $\alpha^{\prime}$ and has the same Morse complex as it has with respect to $\alpha+d \tau^{2}$ (in fact, the set of such metrics, as a countable intersection of open dense sets, is even dense in the mentioned 
neighborhood of $\alpha)$. It will be convenient to use the convention that $n^{t}(p, q)=0$ whenever the difference of indexes between the critical points $p$ and $q$ of a MorseSmale function $t$ is strictly greater than 1 , or if one of $p, q$ is not a critical point of $t$.

Lemma 1.31. In the setting above there is some $n \in \mathbf{N}$ such that in the Morse complex of $F^{1 / n}$ we have:

(i) $n^{F^{1 / n}}(p, q)=n^{F}(p, q)$ if $p, q \in \operatorname{Crit}\left(F^{1 / n}\right) \bigcap \operatorname{Crit}(F), p \in M \times\{1\}$.

(ii) $n^{F^{1 / n}}(p, q)=0$ for $p, q \in \operatorname{Crit}\left(F^{1 / n}\right)$, with $p \in M \times\{1\}$ and $q \in N_{1 /(2 n)}$.

(iii) $n^{F^{1 / n}}(p, q)=0$ for $p, q \in \operatorname{Crit}\left(F^{1 / n}\right)$ with $p \in M \times\{1\}$, $q \in N_{-1 /(2 n)} \cup N_{1 /(2 n)}$ and $F^{1 / n}(p)<0$.

Assuming this lemma, here is the end of the proof of the gluing Theorem 1.29. To simplify notation we denote $G=F^{1 / n}, h=f_{1 / n}$ with $n$ satisfying the conclusion of 1.31. Denote by $\bar{F}, \bar{G}$ respectively the chain maps $F^{M \times[0,1]}$ and $G^{M \times[0,1]}$. If $x \in M \times\{0\} \subset \operatorname{Crit}(f) \bigcap M \times[0,1]$ we let $x^{\prime}=x \times\{1\}$. As $F$ is a linear cobordism we have $\bar{F}\left(x^{\prime}\right)=x$. Consider now the function $h$. It restricts to a linear cobordism on $W(-1 /(2 n), 1 /(2 n))$. If $y \in \operatorname{Crit}(h) \bigcap N_{-1 /(2 n)}$, then let $\theta y=h^{W(-1 /(2 n), 1 /(2 n))}(y)$. Clearly,

$$
\theta y=\bar{u} \times\{1 /(2 n)\}, \quad y=\bar{u} \times\{-1 /(2 n)\}
$$

with $\bar{u}$ a critical point of $g$. Assume $x, y \in \operatorname{Crit}(f)$ are such that $f(x)>0$ and $f(y)<0$ and $i=\operatorname{ind}(x)=\operatorname{ind}(y)+1$. Then

$$
n^{f, \alpha}(x, y)=n^{F, \alpha}\left(x^{\prime}, y^{\prime}\right)=n^{G, \alpha^{\prime}}\left(x^{\prime}, y^{\prime}\right) .
$$

All the counting of flow lines below will be done with respect to the metric $\alpha^{\prime}$ which we shall omit from the terminology.

As $\bar{G}$ is a chain map and in view of the definition of the Morse complex we have

$$
n^{G}\left(x^{\prime}, y^{\prime}\right) n^{G}\left(y^{\prime}, y\right)+\sum_{z \in \operatorname{Crit}_{i}(G), z \neq y^{\prime}} n^{G}\left(x^{\prime}, z\right) n^{G}(z, y)=0 .
$$

Now Lemma 1.22 implies that:

if $z \in M \times\{1\}, z \neq y^{\prime}$ then $n^{G}(z, y)=0$;

if $z \in(M \times\{0\}) \backslash\left(N_{1 /(2 n)} \cup N_{-1 /(2 n)}\right)$ then $n^{G}\left(x^{\prime}, z\right)=0$ except for $z=x$ and then $n^{G}\left(x^{\prime}, x\right)=1$;

if $z \in N_{-1 /(2 n)} \cup N_{1 /(2 n)}$ then $n^{G}\left(y^{\prime}, z\right)=0$;

if $z \in N_{1 /(2 n)}$ then $n^{G}\left(x^{\prime}, z\right)=0$.

As also $n^{G}\left(y^{\prime}, y\right)=1, n^{G}(x, y)=0$ we obtain

$$
n^{G}\left(x^{\prime}, y^{\prime}\right)+\sum_{z \in N_{-1 /(2 n)}} n^{G}\left(x^{\prime}, z\right) n^{G}(z, y)=0 .
$$


Moreover for each $z \in N_{-1 / 2 n}$, we also have

$$
n^{G}\left(x^{\prime}, z\right) n^{G}(z, \theta z)+\sum_{v \in \operatorname{Crit}_{i}(G)} n^{G}\left(x^{\prime}, v\right) n^{G}(v, \theta z)=0
$$

which gives

$$
n^{G}\left(x^{\prime}, z\right)+n^{G}(x, \theta z)=0 .
$$

So we get

$$
\begin{aligned}
n^{f}(x, y) & =n^{G}\left(x^{\prime}, y^{\prime}\right) \\
& =\sum_{\bar{z} \in \operatorname{Crit}_{\mathrm{i}-1}(g)} n^{G}(z, y) n^{G}(x, \theta z) \\
& =\sum_{\bar{z} \in \mathrm{Crit}_{\mathrm{i}-1}(g)} n^{h}(z, y) n^{h}(x, \theta z) .
\end{aligned}
$$

We can now define

$$
h^{\prime}=\left.h\right|_{f^{-1}(-\infty,-1 /(2 n)]}, h^{\prime \prime}=\left.h\right|_{f^{-1}[1 /(2 n), \infty)}
$$

and modulo the obvious identification of $N_{-1 /(2 n)}$ with $N_{1 /(2 n)}$ we obtain the desired formula.

It remains now to prove the lemma.

Proof of Lemma 1.31. We fix $p \in \mathrm{Crit}_{i}(F) \bigcap M \times\{1\}$. We have $p=p_{1} \times\{1\}$ with $p_{1} \in \mathrm{Crit}_{i-1}(F) \bigcap M \times\{0\}$. Consider $q \in \mathrm{Crit}_{i-1}(F) \bigcap M \times\{0\}$. We have $n^{F}(p, q)=0$ if $q \neq p_{1}$ and $n^{F}(p, q)=1$ if $q=p_{1}$. We shall now consider two cases:

(a) $F(p)<0$. In this case, by choosing $n$ sufficiently large, we have

$$
F(p)=F^{1 / n}(p) \leq \inf \left(F^{1 / n}\left(M^{1 / n}\right)\right)
$$

and therefore $W_{F^{1 / n}}^{u} \bigcap M^{1 / n}=\emptyset$, so that

$$
\begin{aligned}
& n^{F^{1 / n}}(p, q)=n^{F}(p, q), \\
& n^{F^{1 / n}}(p, r)=0 \text { for all } r \in N_{-1 /(2 n)} \bigcup N_{1 /(2 n)} .
\end{aligned}
$$

As the number of critical points of $f$ is finite this implies that for a sufficiently large $n$ the three properties of Lemma 1.22 are verified for all critical points $p$ such that $F(p)<0$.

(b) $F(p)>0$. The same argument as above shows that for all $q$ with $f(q)>0$ we have that for sufficiently large $n$

$$
n^{F^{1 / n}}(p, q)=n^{F}(p, q)
$$

There are two other properties that we still have to verify (and it is only here that we shall need to use the specific form of $\left.F^{1 / n}\right)$ :

(1) if $f(q)<0$, then $n^{F^{1 / n}}(p, q)=0$,

(2) if $r \in N_{1 /(2 n)}$, then $n^{F^{1 / n}}(p, r)=0$. 
We first note that for each $\epsilon$ the boundary of $M^{\epsilon}$ has the property that $W^{(-\epsilon, \epsilon)} \times$ $\left\{5 \epsilon^{1 / 3}\right\} \bigcup N_{\epsilon} \times\left[0,5 \epsilon^{1 / 3}\right]$ is an entrance set into $M^{\epsilon}$ for the flow induced by the negative of the gradient of $F^{\epsilon}$. Similarly, $N_{-\epsilon} \times\left[0,5 \epsilon^{1 / 3}\right]$ is an exit set. Assume that for arbitrarily large $n$ the property (1) above is not satisfied. As the number of critical points of $f$ is finite this means that there is a critical point $q$ of $f$ of index $i-1$ and a sequence $n_{k} \rightarrow \infty$ such that $n^{F^{1 / n_{k}}}(p, q) \neq 0$ with $f(q)<0$. Clearly, as $n^{F}(p, q)=0$ this means that for each $n_{k}$ there is at least one flow line $\lambda_{k}$ (for the flow induced by $F^{1 / n_{k}}$ ) that joins $p$ to $q$ and that intersects $M^{1 / n_{k}}$. Let $x_{k}$ be the entrance point of this flow line into $M^{1 / n_{k}}$ and let $y_{k}$ be the exit point of this same flow line. Inside $M^{1 / n_{k}}$ the flow line joining $x_{k}$ to $y_{k}$ projects onto $N=N_{0}$ as a flow line $\lambda_{k}^{\prime}$ of $g$ joining the projection of $x_{k}$ denoted by $x_{k}^{\prime}$ to the projection $y_{k}^{\prime}$ of $y_{k}$. This happens because the gradient of $F^{1 / n_{k}}$ can be decomposed into two components, one orthogonal to $N$ and the other having the same direction as the gradient of $g$. We now make $k \rightarrow \infty$. Then, by compactness, we may assume that $x_{k} \rightarrow x_{0} \in N, y_{k} \rightarrow y_{0} \in N$. As in the exterior of $M^{1 / n_{k}}$ the function $F^{1 / n_{k}}$ coincides with $F$ we obtain that $y_{0} \in \bar{W}_{F}^{s}(q)$ and $x_{0} \in \bar{W}_{F}^{u}(p)$. Because $F$ is a linear cobordism this implies $x_{0} \in \bar{W}_{f}^{u}\left(p_{1}\right)$ and $y_{0} \in \bar{W}_{f}^{s}(q)$ (see Remark 1.5 (b)). Of course, we also have $x_{k}^{\prime} \rightarrow x_{0}$ and $y_{k}^{\prime} \rightarrow y_{0}$. Property (i) of the function $g$ implies that $x_{0} \in \bigcup_{z \in \mathrm{Crit}_{\leq i-2}} W_{g}^{u}(z)$. As $x_{k}^{\prime}$ and $y_{k}^{\prime}$ were joined by the flow lines $\lambda_{k}^{\prime}$ of the flow induced by the negative of the gradient of $g$ we obtain that $y_{0}$ also belongs to $\bigcup_{z \in \mathrm{Crit}_{<i-2}} W_{g}^{u}(z)$. But this means that the intersection of some $g$-unstable manifold of dimension $\leq i-2$ with the $f$-stable manifold of $q$ is non trivial. By property (ii) of $g$ that means

$$
\operatorname{dim}(M)-\operatorname{ind}_{f}(q)+(i-2)=\operatorname{dim}\left(W_{f}^{S}(q)\right)+(i-1) \geq \operatorname{dim}(M) .
$$

This implies $\operatorname{ind}(q) \leq i-2$ and leads to a contradiction.

We use a similar argument to deal with condition (2). As above, if for arbitrarily large $n$ (2) is not satisfied there is a critical point of $F^{1 / n_{k}}, r_{k} \in N_{1 /\left(2 n_{k}\right)}$ such that there exists at least one $F^{1 / n_{k}}$-flow line $\lambda_{k}$ joining $p$ to $r_{k}$ and ind $\left(r_{k}\right)=i-1$. Obviously, when $k \rightarrow \infty$ we may assume that $r_{k} \rightarrow r$ with $r$ a critical point of index $i-1$ of $g$. As before, let $x_{k}$ be the entrance point of $\lambda_{k}$ inside $M^{1 / n_{k}}$ and let $x_{k}^{\prime}$ be the projection of $x_{k}$ onto $N$. The portion of $\lambda_{k}$ inside of $M^{1 / n_{k}}$ projects to a $g$-flow line $\lambda_{k}^{\prime}$ joining $x_{k}^{\prime}$ to $r$. Let

$$
x_{0}=\lim \left(x_{k}\right)=\lim \left(x_{k}^{\prime}\right) .
$$

As above we obtain

$$
x_{0} \in \bar{W}_{F}^{u}(p) \bigcap M \times\{0\}=\bar{W}_{f}^{u}\left(p_{1}\right) .
$$

At the same time we also have $x_{0} \in \overline{W_{g}^{S}(r)}$. Property (ii) of $g$ implies

$$
\operatorname{dim}\left(W_{g}^{s}(r)\right)+\operatorname{dim}\left(W_{f}^{u}\left(p_{1}\right)\right) \geq n
$$

which means

$$
n-1-\operatorname{ind}_{g}(r)+\operatorname{ind}_{f}\left(p_{1}\right) \geq n \text {. }
$$


It follows that ind $\operatorname{li}_{g}(r) \leq i-2$, which contradicts the hypothesis on $r$ and completes the proof of the lemma (we have actually proved more: not only the relevant numbers of flow lines counted with signs are zero but actually the respective sets of flow lines are empty), and also of the gluing Theorem 1.29.

\section{Applications}

2.1. Rigidity of the Floer complex. The purpose of this section is to adapt the technique described in Sect. 1.2 to show a rigidity result for the Floer complex which is similar to Theorem 1.19. This adaptation is rather immediate as it only makes use of very standard tools from the construction of Floer homology.

2.1.1. The Floer complex. We start by recalling - sketchily - some elements of the construction of the Floer complex. This construction is presented in detail in many sources and we refer to them for details [18], [9] [19]. We follow closely the last two sources mentioned as our sign conventions are like there.

Let $M$ be a symplectic closed manifold with symplectic form $\omega$ and of dimension $2 m$. We shall work under the assumption that $\omega$ vanishes when evaluated on $\pi_{2}(M)$. There exist almost complex structures $J$ on $M$ that are compatible with $\omega$ in the sense that the bilinear form $g_{J}(X, Y)=\omega(X, J(x) Y), X, Y \in T_{x} M$, gives a Riemannian metric on $M$. In fact, the set of such compatible almost complex structures - which will be denoted by $\mathcal{J}$ - is contractible and therefore there exists a well defined Chern class $c_{1} \in H^{2}(M ; \mathbb{Z})$. We shall also assume $\left.c_{1}\right|_{\pi_{2}(M)}=0$.

Fix a 1-periodic hamiltonian $H$ on $M$. In other words, this is a smooth function $H: S^{1} \times M \rightarrow \mathbb{R}$ where $S^{1}=\mathbb{R} / \mathbb{Z}$. Such a hamiltonian induces a one parameter family of Hamiltonian vector fields $X_{H}^{t}, t \in S^{1}$, on $M$ which is defined by the equality $\omega\left(X_{H}^{t}, Y\right)=-d\left(H_{t}\right)(Y)$ that is required to hold for all vector fields $Y$ on $M$. To this family we associate the time dependent differential equation

$$
\dot{x}(t)=X_{H}^{t}(x(t)) .
$$

The solutions to this equation define a one-parameter family of symplectic diffeomorphisms $\phi^{t}$ on $M$ given by $\phi_{H}^{t}(x(0))=x(t)$.

The problem which is addressed by the Floer complex is to estimate the number of solutions of equation (1) that satisfy $x(1)=x(0)$ and are contractible as loops in $M$. We denote by $\Lambda_{0}$ the space of all smooth, contractible loops in $M$ and we denote by $P(H) \subset \Lambda_{0}$ these 1-periodic solutions.

Let $a_{H}: \Lambda_{0} \rightarrow \mathbb{R}$ be the function defined by

$$
a_{H}(x)=-\int_{D} \bar{x}^{*} \omega+\int_{0}^{1} H(t, x(t)) d t .
$$

Here $\bar{x}: D \rightarrow M$ is an extension of $x: S^{1} \rightarrow M$ to the unit disk $D \subset \mathbb{R}^{2}, S^{1}=$ $\partial D$. Because $\left.\omega\right|_{\pi_{2}(M)}=0$, the first integral in the formula of $a_{H}$ is independent of the choice of the extension $\bar{x}$ and $a_{H}$ is well defined.

The critical points of $a_{H}$ are identified with the elements of $P(H)$ and Floer's theory is a sort of Morse theory for functionals of this type. Of course, a number 
of non-degeneracy conditions are needed. The first - which we shall assume from now on - is that all elements of $P(H)$ are required to be non-degenerate in the sense that

$$
\operatorname{det}\left(1-d \phi_{H}^{1}(x(0))\right) \neq 0
$$

This condition is generically satisfied and is similar to the requirement that a function be Morse in the classical situation. Given that $M$ is compact and non-degenerate periodic orbits are isolated, it follows that the number of elements of $P(H)$ is finite. To each element $x \in P(H)$ we may associate an integer $\mu_{H}(x)=\mu_{C Z}\left(x^{\prime}\right)$ which will play the role of the Morse index in the classical Morse case. Here $\mu_{C Z}\left(x^{\prime}\right)$ is the Conley-Zehnder index [19] of the path $x^{\prime}:[0,1] \rightarrow S p(2 m)$ defined as follows. We first choose $\bar{x}: D \rightarrow M$ extending $x$. We fix a trivialization of the tangent bundle of $M$ over $\operatorname{Im}(\bar{x})$ and we use it to view the family $d\left(\phi_{H}^{t}\right)_{x(0)}$ as a path $x^{\prime}:[0,1] \rightarrow S p(2 m)$ with $x^{\prime}(0)=I d$ and $\operatorname{det}\left(I d-x^{\prime}(1)\right) \neq 0$ (because $x$ is non-degenerate). One important point here is that because $\left.c_{1}\right|_{\pi_{2}(M)}=0$ any two extensions $\bar{x}$ produce homotopic paths $x^{\prime}$ and thus the definition of $\mu_{H}(x)$ is independent of the choice of extension.

The fundamental problem is that the gradient of the functional $a_{H}$ does not define a flow. However, the solutions $u(s, t): \mathbb{R} \times \mathbb{R} / \mathbb{Z} \rightarrow M$ of the equation

$$
\frac{\partial u}{\partial s}+J(u) \frac{\partial u}{\partial t}+\nabla_{x} H(t, u)=0
$$

may be viewed as "negative gradient flow lines" of this functional. Here $J \in \mathcal{J}$ and $\nabla_{x} H$ is the gradient of $H(t,-)$ with respect to the metric $g_{J}$.

The energy of a map $u: \mathbb{R} \times S^{1} \rightarrow M$ is defined by

$$
E_{H}(u)=\frac{1}{2} \int_{-\infty}^{\infty} \int_{0}^{1}\left(\left|\frac{\partial u}{\partial s}\right|^{2}+\left|\frac{\partial u}{\partial t}-X_{H}^{t}(u)\right|^{2}\right) d t d s
$$

We shall denote by $\mathcal{M}(H, J)$ the space of solutions $u$ of the equation 3 that verify $E_{H}(u)<\infty$. This space has the remarkable property to be compact and to decompose as the union of the spaces

$$
\mathcal{M}(y, x ; H, J)=\left\{u \in \mathcal{M}(H, J): \lim _{s \rightarrow-\infty} u(s, t)=y(t), \lim _{s \rightarrow+\infty} u(s, t)=x(t)\right\}
$$

where $x, y \in P(H)$. Here, convergence is uniform in $t$ and, as it should happen for true gradient flow lines of $a_{H}$, the elements of $\mathcal{M}(y, x ; H, J)$ also verify $\lim _{s \rightarrow \pm \infty} \frac{\partial u}{\partial s}(s, t)=0$ uniformly in $t$. Moreover, for $u \in \mathcal{M}(y, x ; H, J)$ we have $E_{H}(u)=a_{H}(y)-a_{H}(x)$. There is an obvious action of $\mathbb{R}$ on the space $\mathcal{M}(y, x ; H, J)$ and we shall denote by $\mathcal{M}^{\prime}(y, x ; H, J)$ the resulting orbit space.

For our fixed almost complex structure $J$ there exists a subset $\mathcal{H}_{\text {reg }}(J)$ of the second Baire category inside $C^{\infty}\left(S^{1} \times M\right)$ such that whenever $H \in \mathcal{H}_{\text {reg }}(J)$ the natural linearization $D_{u, H, J}$ of the operator $\bar{\partial}_{H, J}(u)=\frac{\partial u}{\partial s}+J(u) \frac{\partial u}{\partial t}+\nabla_{x} H(t, u)$ is surjective at each $u$ with $\bar{\partial}_{H, J}(u)=0$ [6]. We shall call the pair $(H, J)$ regular whenever $H$ satisfies (2) and $H \in \mathcal{H}_{\text {reg }}(J)$. In the Morse case, the analogue of the 
condition $H \in \mathcal{H}_{\text {reg }}(J)$ is the Morse-Smale transversality requirement (when the Riemannian metric is fixed).

If $(H, J)$ is a regular pair, the surjectivity of $D_{u, H, J}$ for all solutions $u$ of (3) implies that $\mathcal{M}(y, x ; H, J)$ are manifolds whose dimension can be computed to be $\mu_{H}(y)-\mu_{H}(x)$. Therefore, the spaces $\mathcal{M}^{\prime}(y, x ; H, J)$ are manifolds of dimension $\mu_{H}(y)-\mu_{H}(x)-1$. Moreover, in our setting, these manifolds admit coherent orientations [4]. The spaces $\mathcal{M}^{\prime}(y, x ; H, J)$ also have natural compactifications $\overline{\mathcal{M}^{\prime}}(y, x ; H, J)$ which are manifolds with boundary and whose combinatorics is quite similar to that of the moduli spaces of flow lines in the Morse-Smale case. In particular, if $\mu_{H}(x)=\mu_{H}(y)-1$, then $\mathcal{M}^{\prime}(y, x ; H, J)$ is finite and if $\mu_{H}(x)=$ $\mu_{H}(y)-2$, then we have the (oriented) equality:

$$
\partial \overline{\mathcal{M}^{\prime}}(y, x ; H, J)=\coprod_{\substack{z \in P(H) \\ \mu_{H}(z)=\mu_{H}(y)-1}} \mathcal{M}^{\prime}(y, z ; H, J) \times \mathcal{M}^{\prime}(z, x ; H, J) .
$$

The Floer complex of $(H, J)$ is now defined by:

$$
\begin{array}{r}
C F_{i}(H, J)=\mathbb{Z}\left[x \in P(H): \mu_{H}(x)=i\right], d: C F_{i}(H, J) \rightarrow C F_{i-1}(H, J), \\
d(x)=\sum_{z \in P(H), \mu_{H}(z)=\mu_{H}-1} n^{H, J}(x, z)
\end{array}
$$

where $n^{H, J}(x, z)$ is the number of elements (counted with signs) of $\mathcal{M}^{\prime}(x, z ; H, J)$.

\subsubsection{Rigidity. For a fixed hamiltonian $H: S^{1} \times M \rightarrow \mathbb{R}$ let}

$$
\delta_{H}=\inf \left\{a_{H}(y)-a_{H}(x): x, y \in P(H), \mathcal{M}(y, x ; H, J) \neq \emptyset\right\} .
$$

When all the 1-periodic orbits of $X_{H}$ are non-degenerate the set $P(H)$ is finite and we have $\delta>0$. The purpose of this section is to prove the following result.

Theorem 2.1. Fix a regular pair $(H, J)$. If $\left(H^{\prime}, J^{\prime}\right)$ is another regular pair with $\left\|H^{\prime}-H\right\|_{0} \leq \delta_{H} / 4$, then the Floer complex $C F_{*}(H, J)$ is a retract of the complex $C F_{*}\left(H^{\prime}, J^{\prime}\right)$.

As in the Morse case, we immediately deduce.

Corollary 2.2. Given a Hamiltonian $H: M \rightarrow \mathbb{R}$ let $J, J^{\prime}$ be two almost complex structures such that the pairs $(H, J)$ and $\left(H, J^{\prime}\right)$ are regular. There exists an isomorphism of chain complexes $C F_{*}(H, J) \approx C F_{*}\left(H, J^{\prime}\right)$.

Proof. The proof of the theorem is based on the idea described in 1.2 and, besides the usual approach to comparing the Floer complexes of two different regular pairs, it makes use of monotone homotopies as introduced in [5], [2].

The standard way to compare the Floer complexes associated to the two regular pairs $\left(H^{0}, J^{0}\right)$ and $\left(H^{1}, J^{1}\right)$ is as follows [19] [9]. Take smooth homotopies $H^{01}$ : $\mathbb{R} \times S^{1} \times M \rightarrow \mathbb{R}$ and $J^{01}: \mathbb{R} \times M \rightarrow \operatorname{End}(T M), J_{s}^{01} \in \mathcal{J}, \forall s \in \mathbb{R}$ such that there exists $R>0$ with the property that, for $s \geq R$, we have $\left(H_{s}^{01}(x), J_{s}^{01}(x)\right)=$ 
$\left(H^{0}(x), J^{0}(x)\right)$ and for $s \leq-R,\left(H_{s}^{01}, J_{s}^{01}\right)=\left(H^{1}(x), J^{1}(x)\right), \forall x \in M$. Consider the equation:

$$
\frac{\partial u}{\partial s}+J^{01}(s, u) \frac{\partial u}{\partial t}+\nabla_{x}^{s} H^{01}(s, t, u)=0
$$

where $\nabla_{x}^{s} H^{01}(s, t,-)$ is the gradient of the function $H(s, t,-)$ with respect to the Riemannian metric induced by $J_{s}^{01}$. Let $\mathcal{M}\left(H^{01}, J^{01}\right)$ be the space of all finite energy solutions $u: \mathbb{R} \times \mathbb{R} / \mathbb{Z} \rightarrow M$ of this equation (where the energy $E_{H^{01}}$ is defined by using $H^{01}$ in (4) instead of $H$ ). Again this space is compact and it decomposes as the union of the spaces $\mathcal{M}\left(y, x ; H^{01}, J^{01}\right)$ containing those solutions $u$ that also satisfy

$$
\lim _{s \rightarrow-\infty} u(s, t)=y(t), \lim _{s \rightarrow+\infty} u(s, t)=x(t)
$$

for some with $x \in P\left(H^{0}\right)$ and $y \in P\left(H^{1}\right)$. Moreover, for generic choices of $\left(H^{01}, J^{01}\right)$, the associated linearized operator $D_{u, H^{01}, J^{01}}$ is surjective for each solution $u$ of (5). Whenever $\left(H^{i}, J^{i}\right)$ are regular pairs for $i \in\{0,1\}$ and this additional condition is satisfied we shall say that the pair $\left(H^{01}, J^{01}\right)$ is a regular homotopy. As in the case of equation (3) a consequence of regularity is that the spaces $\mathcal{M}\left(y, x ; H^{01}, J^{01}\right)$ are manifolds and they also admit coherent orientations. The dimension of such a manifold is given by $\mu_{H^{1}}(y)-\mu_{H^{0}}(x)$. Define now $\psi^{H^{01}, J^{01}}: C F_{*}\left(H^{1}, J^{1}\right) \rightarrow C F_{*}\left(H^{0}, J^{0}\right)$ by

$$
\psi^{H^{01}, J^{01}}(y)=\sum_{x \in P\left(H^{0}\right), \mu_{H^{0}}(x)=\mu_{H^{1}}(y)} n^{H^{01}, J^{01}}(y, x)
$$

where $n^{H^{01}, J^{01}}(y, x)$ is the number of elements in $\mathcal{M}\left(y, x ; H^{01}, J^{01}\right)$ (counted with signs). Obviously, the key point is that this application is a chain homomorphism which induces an isomorphism in homology ([19], [18]). For such a homotopy $H^{01}$ let

$$
a_{H^{01}}(s, x)=-\int_{D} \bar{x}^{*} \omega+\int_{0}^{1} H^{01}(s, t, x(t)) d t .
$$

We now consider $u: \mathbb{R} \rightarrow \Lambda_{0}, u \in \mathcal{M}\left(y, x, H^{01}, J^{01}\right)$ and by composition define $a(s)=a_{H^{01}}(s, u(s))$. We derive $a(s)$ with respect to $s$ and we obtain (as, for example, in [5] p. 50 - except for a change in signs)

$$
\frac{d a}{d s}(s)=d\left(a_{H_{s}^{01}}\right)(u(s, t)) \frac{\partial u}{\partial s}(s, t)+\int_{0}^{1} \frac{\partial H^{01}}{\partial s}(s, t, u(s, t)) d t
$$

where $d\left(a_{H}\right)(\gamma) \xi$ is the derivative of the functional $a_{H}: \Lambda_{0} \rightarrow \mathbb{R}$ at $\gamma \in \Lambda_{0}$ in the direction of the vector field $\xi$ defined along $\gamma$. Now

$$
\begin{aligned}
& d\left(a_{H_{s}^{01}}\right)(u(s, t)) \frac{\partial u}{\partial s}(s, t)= \\
& =\int_{0}^{1} g_{J_{s}^{01}}\left(J^{01}(s, u(s, t)) \frac{\partial u}{\partial t}(s, t)+\nabla_{x}^{s} H^{01}(s, t, u(s, t)), \frac{\partial u}{\partial s}(s, t)\right) d t
\end{aligned}
$$

and, because $u$ verifies equation (5), this is always negative. 
If it happens that $H^{1}(t, x)>H^{0}(t, x)$ for all $(t, x) \in S^{1} \times M$ we may take $H^{10}$ to be a monotone homotopy in the sense that $\frac{\partial H^{01}}{\partial s}(s, t, x) \leq 0$ for all $(s, t, x) \in \mathbb{R} \times S^{1} \times M$ (this is quite analogue to our simple morse cobordisms from Definition 1.10). These homotopies have been introduced and used by Floer and Hofer in [5]. If $H^{01}$ is monotone, the formula above shows that $\frac{d a}{d s} \leq 0$ which shows that

$$
a_{H^{1}}(y)=a_{H^{10}}(-R, y) \geq a_{H^{10}}(R, x)=a_{H^{0}}(x) .
$$

Suppose now that we have two regular homotopies $\left(H^{i(i+1)}, J^{i(i+1)}\right), i \in\{0,1\}$ relating the regular pairs $\left(H^{i}, J^{i}\right)$ and $\left(H^{i+1}, J^{i+1}\right)$. It is then possible to glue the two homotopies together thus getting a new homotopy $\left(H^{02}, J^{02}\right)$ which is defined by $H_{T}^{02}(s, t, x)=H^{01}(s+T, t, x)$ for $s \leq 0, H_{T}^{02}(s, t, x)=H^{12}(s-T, t, x)$ for $s \geq 0$ and by analogue formulae for $J^{02}$. For $T$ sufficiently big this homotopy is regular and it verifies $\psi^{H^{02}, J^{02}}=\psi^{H^{01}, J^{01}} \circ \psi^{H^{12}, J^{12}}$ ([19], Lemma 6.4; [18] Lemma 3.11). Notice also that if both $H^{01}$ and $H^{12}$ are monotone, then so is $H^{02}$.

We now turn to the statement of the theorem. We fix the regular pair $(H, J)$ and we let $\left(H^{\prime}, J^{\prime}\right)$ be a regular pair with $\left|H^{\prime}(t, x)-H(t, x)\right| \leq \delta_{H} / 4, \forall(t, x) \in S^{1} \times M$. We have $H(t, x)-\delta_{H} / 3<H^{\prime}(t, x)<H(t, x)+\delta_{H} / 3, \forall(t, x) \in S^{1} \times M$. Let $\left(H^{2}, J^{2}\right)=\left(H+\delta_{H} / 3, J\right),\left(H^{1}, J^{1}\right)=\left(H^{\prime}, J^{\prime}\right),\left(H^{0}, J^{0}\right)=\left(H-\delta_{H} / 3, J\right)$. We may find monotone homotopies $\left(H^{01}, J^{01}\right),\left(H^{12}, J^{12}\right)$ relating these pairs such that for all $s \in \mathbb{R}$ we have $H^{0} \leq H_{s}^{01} \leq H^{1} \leq H_{s}^{12} \leq H^{2}$. For fixed $H^{i j}$ the pair $\left(H^{i j}, J^{i j}\right)$ is regular for a generic choice of $J^{i j}$ inside the set of time-dependent, period one, almost complex structures compatible with $\omega$, [5] Theorem 23 (the use of time dependent almost complex structures does not change the form or the behaviour of any of the previous equations).

We consider the "glued" regular homotopy $\left(H^{02}, J^{02}\right)=\left(H_{T}^{02}, J_{T}^{02}\right)$ defined as above which is monotone and regular for large $T$.

In essence, we have now constructed the two non-trivial faces of the cube in the proof of Theorem 1.19 and, to end the proof, we need to show that $\psi^{H^{02}, J^{02}}$ is an isomorphism. We consider the monotone homotopy $\left(\bar{H}^{02}, J\right)$ such that $H^{02}(s, t, x)=H(t, x)+h(s)$ with $h: \mathbb{R} \rightarrow \mathbb{R}$ some convenient, decreasing function and $J_{s}=J$. Obviously, this homotopy is regular and $\psi^{\bar{H}^{02}, J}=i d$. Let $(G, \bar{J})$ be a smooth homotopy of homotopies $G:[0,1] \times \mathbb{R} \times S^{1} \times M \rightarrow \mathbb{R}$ such that $G_{\lambda}=G(\lambda,-,-,-)$ verifies $G_{0}=H^{02}, G_{1}=\bar{H}^{02}$ and we have similar identities for $\bar{J}$. There exists a notion of regularity for such a homotopy of homotopies $(G, \bar{J})$. If our $(G, \bar{J})$ is regular in this sense it induces a chain homotopy $\Psi=\psi^{G, \bar{J}}: C F_{*}\left(H^{2}, J^{2}\right) \rightarrow C F_{*+1}\left(H^{0}, J^{0}\right)$ defined by

$$
\psi^{G, \bar{J}}(y)=\sum_{x \in P\left(H^{0}\right), \mu_{H^{0}}(x)=\mu_{H^{2}}(y)+1} n^{G, \bar{J}}(y, x)
$$

where $n^{G, \bar{J}}(y, x)$ is the number of elements (counted with signs) of the moduli spaces

$$
\mathcal{M}(y, x ; G, \bar{J})=\left\{(u, \lambda): \lambda \in[0,1], u \in \mathcal{M}\left(y, x ; G_{\lambda}, \bar{J}_{\lambda}\right)\right\}
$$


which, are finite by regularity when $\mu_{H^{0}}(x)=\mu_{H^{2}}(y)+1$ and also admit a coherent choice of orientations (see [19] Lemma 6.3, [5] Sect. 4.3). With this definition we have $d \Psi-\Psi d=\psi^{\bar{H}^{02}, J}-\psi^{H^{02}, J^{02}}=I d-\psi^{H^{02}, J^{02}}$. We index the elements in $P\left(H^{2}\right)=P\left(H^{0}\right)=P(H)=\left\{p_{1}, p_{2}, \ldots p_{k}\right\}$ such that $a_{H}\left(p_{i}\right)<a_{H}\left(p_{j}\right) \Rightarrow i<j$ and we denote by $A=\left(a_{i j}\right)$ the matrix of the linear map $d \Psi-\Psi d$ in this basis.

Because both $H^{02}$ and $\bar{H}^{02}$ are monotone homotopies we may take $G$ to be a homotopy of monotone homotopies in the sense that $G_{\lambda}$ is a monotone homotopy for all $\lambda \in[0,1]$. Simultaneously, we also need the pair $(G, \bar{J})$ to be regular. This can be achieved generically (with again $\bar{J}$ time-dependent) as discussed in [5] Sect. 4.3. In this case, it follows from (7) that $n^{G, \bar{J}}(y, x) \neq 0 \Rightarrow$ $a_{H^{2}}(y) \geq a_{H^{0}}(x)$. The coefficients of the Floer differential in $C F_{*}(H, J)$ verify $n^{H, J}(y, x) \neq 0 \Rightarrow a_{H}(y) \geq \delta_{H}+a_{H}(x)$. By looking to the matrix $A$ we obtain that $a_{i j} \neq 0 \Rightarrow a_{H^{2}}\left(p_{j}\right) \geq a_{H^{0}}\left(p_{i}\right)+\delta_{H}$. But $a_{H}\left(p_{j}\right)+\delta_{H} / 3=a_{H^{2}}\left(p_{j}\right)$, $a_{H^{0}}\left(p_{i}\right)=a_{H}\left(p_{i}\right)-\delta_{H} / 3$ so we get that if $a_{i j} \neq 0$, then $a_{H}\left(p_{j}\right)>a_{H}\left(p_{i}\right)$ which means $j>i$. Therefore, $A$ is upper triangular and we conclude that $\psi^{H^{02}, J^{02}}$ is an isomorphism which ends the proof.

Remark 2.3. It is clear that the result above can be extended in many ways. One such possibility is to consider monotone symplectic manifolds which means that the two morphisms $c_{1}: \pi_{2}(M) \rightarrow \mathbb{Z}$ and $[\omega]: \pi_{2}(M) \rightarrow \mathbb{R}$ are proportional. Besides other technical complications, this implies that the action-functional $a_{H}$ is $S^{1}$ valued. In the next section we discuss rigidity (and gluing) for $S^{1}$ valued Morse functions. It is quite likely that the rigidity statement proved below in the $S^{1}$-valued case carries over to the Floer monotone setting.

2.2. Circle-valued functions. The purpose of this subsection is to apply the methods of Sect. 1 to $S^{1}$-valued functions. We shall obtain a rigidity statement similar to that in 1.2 and we shall also adapt to this context the gluing result 1.29. This is then used to show how to algebraically recompose the Morse-Novikov complex out of information on a single fundamental domain.

2.2.1. The Novikov complex. We now consider a circle-valued Morse-Smale function on a compact manifold $M$

$$
(f, \alpha): M \rightarrow S^{1}=\mathbb{R} / \mathbb{Z}
$$

In this section we fix the main definitions and properties of the Novikov complex of $f$.

The pullback infinite cyclic cover

$$
\bar{M}=f^{*} \mathbb{R}=\left\{(x, t) \in M \times \mathbb{R} \mid f(x)=[t] \in S^{1}\right\}
$$

is non-compact, and $(f, \alpha)$ lifts to a $\mathbb{Z}$-equivariant Morse-Smale function

$$
(\bar{f}, \alpha): \bar{M}=f^{*} \mathbb{R} \rightarrow \mathbb{R}
$$


with

$$
\bar{f}: \bar{M} \rightarrow \mathbb{R} ;(x, t) \mapsto t
$$

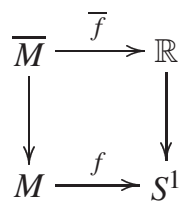

and where we identify the metric $\alpha$ with its pull-back to $\bar{M}$. The generating covering translation along the downward gradient flow of $\bar{f}$ is given by

$$
z: \bar{M} \rightarrow \bar{M} ;(x, t) \mapsto(x, t-1) .
$$

We shall assume that $M$ and $\bar{M}$ are connected, so that there is defined an exact sequence of groups

$$
\{1\} \longrightarrow \pi_{1}(\bar{M}) \longrightarrow \pi_{1}(M) \stackrel{f_{*}}{\longrightarrow} \pi_{1}\left(S^{1}\right)=\mathbb{Z} \longrightarrow\{1\} .
$$

Let $\widetilde{M}$ be a regular cover of $\bar{M}$ with group of covering translations $\pi$ (e.g. $\bar{M}$ itself with $\pi=\{1\}$, or the universal cover of $M$ with $\left.\pi=\pi_{1}(\bar{M})\right)$. Then $\tilde{M}$ is a regular cover of $M$, and the group of covering translations $\Pi$ fits into an exact sequence

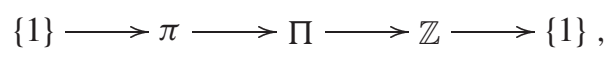

so that

$$
\Pi=\pi \times_{\zeta} \mathbb{Z}
$$

with $\zeta$ the monodromy automorphism defined by

$$
\zeta: \pi \rightarrow \pi ; g \mapsto z^{-1} g z
$$

for any lift of a generator $1 \in \mathbb{Z}$ to an element $z \in \Pi$. The group ring

$$
\mathbb{Z}[\Pi]=\mathbb{Z}[\pi]_{\zeta}\left[z, z^{-1}\right]
$$

consists of the polynomials

$$
\sum_{j=-\infty}^{\infty} a_{j} z^{j} \quad\left(a_{j} \in \mathbb{Z}[\pi]\right)
$$

with $\left\{j \in \mathbb{Z}: a_{j} \neq 0\right\}$ finite and

$$
a z=z \zeta(a)(a \in \mathbb{Z}[\pi])
$$


For any $\mathbb{Z}[\pi]$-module $F$ and $j \in \mathbb{Z}$ let $z^{j} F$ be the $\mathbb{Z}[\pi]$-module with elements $z^{j} x$ $(x \in F)$ and

$$
z^{j} x+z^{j} y=z^{j}(x+y), a z^{j} x=z^{j} \zeta^{j}(a) x \in z^{j} F
$$

Let

$$
(\bar{f}, \alpha): \bar{M} \rightarrow \mathbb{R}
$$

be a lift of $(f, \alpha)$ to a $\mathbb{Z}$-equivariant real-valued Morse function on the infinite cyclic cover $\bar{M}$ of $M$. Lift each critical point of $f: M \rightarrow S^{1}$ to a critical point of $\bar{f}: \bar{M} \rightarrow \mathbb{R}$, allowing the identification

$$
\operatorname{Crit}_{i}(\bar{f})=\bigcup_{j=-\infty}^{\infty} z^{j} \operatorname{Crit}_{i}(f) .
$$

Definition 2.4. (i) The Novikov ring

$$
\mathbb{Z}[\pi]_{\zeta}((z))=\mathbb{Z}[\pi]_{\zeta}[[z]]\left[z^{-1}\right]
$$

is the ring of formal power series

$$
\sum_{j=-\infty}^{\infty} a_{j} z^{j} \quad\left(a_{j} \in \mathbb{Z}[\pi]\right)
$$

such that $\left\{j \leq 0: a_{j} \neq 0\right\}$ is finite.

(ii) The Novikov complex of $(f, \alpha)$ is the based f.g. free $\mathbb{Z}[\pi]_{\zeta}((z))$-module chain complex $C=C^{N o v}(M, f, \alpha)$ with

$$
\begin{gathered}
d: C_{i}=\mathbb{Z}[\pi]_{\zeta}((z))\left[\operatorname{Crit}_{i}(f)\right] \rightarrow C_{i-1}=\mathbb{Z}[\pi]_{\zeta}((z))\left[\operatorname{Crit}_{i-1}(f)\right] ; \\
x \mapsto \sum_{j=-\infty}^{\infty} \sum_{g \in \pi} \sum_{y \in \operatorname{Crit}_{i-1}(f)} n^{\widetilde{f}, \alpha}\left(x, z^{j} g y\right) z^{j} g y .
\end{gathered}
$$

Assume that $1 \in S^{1}$ is a regular value of $f$ and let $N=f^{-1}(1) \subset M$. Cutting $M$ along $N$ gives a fundamental domain $\left(M_{N} ; N, z^{-1} N\right)$ for $\bar{M}$ with a Morse-Smale function

$$
\left(f_{N}, \alpha_{N}\right):\left(M_{N} ; N, z^{-1} N\right) \rightarrow([0,1] ;\{0\},\{1\})
$$

which is constant on $N$ and $z^{-1} N$.

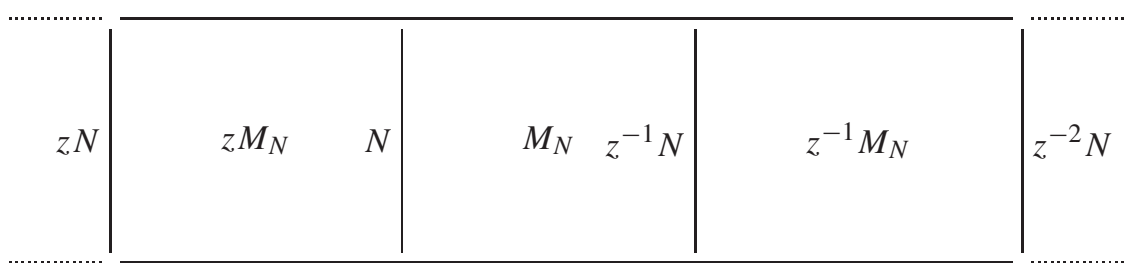


Each critical point $x \in M$ of $f$ has a unique lift to a critical point of $f_{N}$, which is also denoted by $x \in M_{N}$, with

$$
\begin{aligned}
& \bar{f}=\bigcup_{j=-\infty}^{\infty} z^{j} f_{N}: \bar{M}=\bigcup_{j=-\infty}^{\infty} z^{j} M_{N} \rightarrow \mathbb{R}, \\
& \operatorname{Crit}_{i}\left(f_{N}\right)=\operatorname{Crit}_{i}(f), \operatorname{Crit}_{i}(\bar{f})=\bigcup_{j=-\infty}^{\infty} z^{j} \operatorname{Crit}_{i}\left(f_{N}\right), \\
& n^{\bar{f}, \alpha}\left(z^{j} x, z^{k} y\right)=\left\{\begin{array}{ll}
n^{f, \alpha}(x, y) & \text { if } j=k \\
0 & \text { if } j>k
\end{array} \quad\left(x \in \operatorname{Crit}_{i}(f), y \in \operatorname{Crit}_{i-1}(f)\right) .\right.
\end{aligned}
$$

Use the lifts of the critical points of $f$ to $M_{N}$ in the construction of the Novikov complex $C^{N o v}(M, f, \alpha)$, so that the coefficients of the differentials are in $\mathbb{Z}[\pi]_{\zeta}[[z]] \subset$ $\mathbb{Z}[\pi]_{\zeta}((z))$.

Lemma 2.5. The Novikov complex of $(f, \alpha): M \rightarrow S^{1}$ is

$$
C^{N o v}(M, f, \alpha)=\mathbb{Z}[\pi]_{\zeta}((z)) \otimes_{\mathbb{Z}[\pi]_{\zeta}[[z]]}{\underset{\ell}{\lim }}_{\ell} C\left(M_{N}(\ell), f_{N}(\ell), \alpha_{N}(\ell)\right)
$$

with $C\left(M_{N}(\ell), f_{N}(\ell), \alpha_{N}(\ell)\right)$ the $\mathbb{Z}[\pi]$-coefficient Morse complex of the MorseSmale function

$$
\left(f_{N}(\ell), \alpha_{N}(\ell)\right)=(\bar{f}, \alpha) \mid:\left(M_{N}(\ell) ; z^{\ell} N, z^{-1} N\right) \rightarrow \mathbb{R}
$$

on

$$
M_{N}(\ell)=\bigcup_{j=0}^{\ell} z^{j} M_{N} \subset \bar{M}=\bigcup_{j=-\infty}^{\infty} z^{j} M_{N}
$$

The inverse limit is a based f.g. free $\mathbb{Z}[\pi]_{\zeta}[[z]]$-module chain complex, where the limit is with respect to the natural projections

$$
C\left(M_{N}(\ell+1), f_{N}(\ell+1), \alpha_{N}(\ell+1)\right) \rightarrow C\left(M_{N}(\ell), f_{N}(\ell), \alpha_{N}(\ell)\right) .
$$

Proof. Let $F_{i}$ be the based f.g. free $\mathbb{Z}[\pi]$-module generated by the index $i$ critical points of $f$. For any $\ell \geq 0$

$$
C\left(M_{N}(\ell), f_{N}(\ell), \alpha_{N}(\ell)\right)_{i}=\sum_{j=0}^{\ell} z^{j} F_{i}
$$

and

$$
\lim _{\ell} C\left(M_{N}(\ell), f_{N}(\ell), \alpha_{N}(\ell)\right)_{i}=\lim _{\ell} \sum_{j=0}^{\ell} z^{j} F_{i}=\left(F_{i}\right)_{\zeta}[[z]]
$$

so that

$$
\begin{aligned}
& C^{N o v}(M, f, \alpha)_{i}=\left(F_{i}\right)_{\zeta}((z)) \\
& =\mathbb{Z}[\pi]_{\zeta}((z)) \otimes_{\mathbb{Z}[\pi]_{\zeta}[[z]]}{\underset{\lim }{\ell}} C\left(M_{N}(\ell), f_{N}(\ell), \alpha_{N}(\ell)\right)_{i} .
\end{aligned}
$$


2.2.2. Assembly and rigidity for the Novikov complex. It is clear that the Novikov complex of $(f, \alpha): M \rightarrow S^{1}$ only depends on the geometric behaviour of the lift $\bar{f}$ on a single fundamental domain. With the notations of the last section, one expects therefore that there should exist an assembly process that would consist in extracting from $f_{N}$ (or, equivalently, from $\left.f\right|_{M \backslash U(N)}$ with $U(N)$ a small tubular neighbourhood of $N$ ) precisely the data necessary to algebraically piece together $C^{N o v}(M, f, \alpha)$. The work of Pajitnov [16] describes one such method. We shall present below a different one which is based on the techniques described in the first parts of the paper. We will compare in more detail our results with those of Pajitnov at the end of the section.

The notion of a splitting of a real-valued Morse function has an obvious analogue for circle-valued Morse functions. As in the real valued case (see just above Definition 1.26), there is no restriction to assume that $\alpha$ equals the product metric $\beta+d t^{2}$ in a tubular neighbourhood of $N$ with $\beta=\left.\alpha\right|_{N}$.

Definition 2.6. A $(\epsilon, \tau)$-splitting of a Morse-Smale function $(f, \alpha): M \rightarrow S^{1}$ along a Morse-Smale function $(g, \beta): N=f^{-1}(1) \rightarrow \mathbb{R}$ with $\beta=\left.\alpha\right|_{N}$ is a Morse-Smale function $(h, \alpha): M \rightarrow S^{1}$ such that:

(i) $\left.h\right|_{N}=e^{2 \pi i \tau} g: N \rightarrow J$ for a convenient interval $J \subset S^{1}$.

(ii) $(h, \alpha)=(f, \alpha)$ except in a small tubular neighbourhood $W=N \times[-\epsilon, \epsilon] \subset M$ of $N=N \times\{0\} \subset M$. We let $M^{\prime}=M \backslash N$.

(iii) With respect to this parametrization $f(x, t)=e^{2 \pi i t} \in J^{\prime}$ for $(x, t) \in N \times$ $(-\epsilon, \epsilon)$ with $J^{\prime} \subset S^{1}$ an interval containing 1 and

$$
\mathrm{Crit}_{i}(h)=\mathrm{Crit}_{i}(f) \cup\left(\mathrm{Crit}_{i-1}(g) \times\{-\epsilon / 2\}\right) \cup\left(\mathrm{Crit}_{i}(g) \times\{\epsilon / 2\}\right) .
$$

(iv) The restrictions of $(h, \alpha)$ to the submanifolds

$$
\begin{aligned}
& W_{\epsilon}=N \times[-\epsilon / 2, \epsilon / 2] \subset W, \\
& M_{\epsilon}^{\prime}=M^{\prime} \backslash(N \times(-\epsilon / 2, \epsilon / 2)) \subset M^{\prime},
\end{aligned}
$$

are Morse-Smale cobordisms

$$
\begin{aligned}
& \left(g_{\epsilon}, \beta_{\epsilon}\right):\left(W_{\epsilon} ; N \times\{\epsilon / 2\}, N \times\{-\epsilon / 2\}\right) \rightarrow J^{\prime \prime}, \\
& \left(h^{\prime}, \alpha^{\prime}\right):\left(M_{\epsilon}^{\prime} ; N \times\{\epsilon / 2\}, N \times\{-\epsilon / 2\}\right) \rightarrow J^{\prime \prime \prime} \subset S^{1}-\{1\}
\end{aligned}
$$

with $J^{\prime \prime} \cup J^{\prime \prime \prime}=S^{1}$.

(v) The cobordism $\left(g_{\epsilon}, \beta_{\epsilon}\right)$ is linear, so that

$$
C\left(W_{\epsilon}, g_{\epsilon}, \beta_{\epsilon}\right)=C(1: C(N, g, \beta) \rightarrow C(N, g, \beta)) .
$$

The key point of the construction is that, by contrast to the Novikov complex of $(f, \alpha)$, the Novikov complex of a splitting $(h, \alpha)$ is very simple because the flow lines of the corresponding lift $\bar{h}$ are never longer than one fundamental domain. Indeed, the only way a flow line of $h$ can cross $N$ is by joining inside $N \times[-\epsilon / 2, \epsilon / 2]$ a critical point contained in $N \times\{-\epsilon / 2\}$ to one in $N \times\{\epsilon / 2\}$. As $h$ restricts to 
a linear cobordism on $N \times[-\epsilon / 2, \epsilon / 2]$ this leads to a simple description for the Novikov complex of $h$.

For any such splitting we may identify the interval $J^{\prime \prime \prime}$ with $[\epsilon / 2,1-\epsilon / 2]$ (such that, in $\left.S^{1}, 1-\epsilon=-\epsilon\right)$ and if we denote $N^{\prime}=N \times\{\epsilon / 2\}$ and $N^{\prime \prime}=N \times\{1-\epsilon / 2\}$, then the cobordism $\left(h^{\prime}, \alpha^{\prime}\right)$ equals $\left.(\bar{h}, \alpha)\right|_{h^{-1}([\epsilon / 2,1-\epsilon / 2])}$. Clearly, $\left(M_{\epsilon}^{\prime} ; N^{\prime}, N^{\prime \prime}\right)$ is diffeomorphic to $\left(M_{N} ; N, z^{-1} N\right)$. The cobordism

$$
\left(h^{\prime}, \alpha_{N}^{\prime}\right)=\left(h_{N}, \alpha_{N}\right) \mid:\left(M_{\epsilon}^{\prime} ; N^{\prime}, N^{\prime \prime}\right) \rightarrow \mathbb{R}
$$

has

$$
\operatorname{Crit}_{i}\left(h^{\prime}\right)=\left(\operatorname{Crit}_{i}(g) \times\{\epsilon / 2\}\right) \cup \operatorname{Crit}_{i}\left(f_{N}\right) \cup\left(\operatorname{Crit}_{i-1}(g) \times\{1-\epsilon / 2\}\right) .
$$

The algebraic data contained in a splitting of an $S^{1}$-valued map is brought together in the following structure.

Definition 2.7. The algebraic cobordism of a splitting $(h, \alpha): M \rightarrow S^{1}$ of $(f, \alpha)$ : $M \rightarrow S^{1}$ along $g$ is defined by

$$
\Gamma_{N}(M, h, \alpha)=\left(F, D, \theta, \theta^{\prime}, \psi\right)
$$

with $\mathbb{Z}[\pi]$-module chain complexes and chain maps

$$
\begin{aligned}
& D=C(N, g, \beta), F=C\left(M_{N}^{\prime} \backslash \partial M_{N}^{\prime}, h_{N}^{\prime}\left|, \alpha_{N}^{\prime}\right|\right)=C\left(M_{N}, f_{N}, \alpha_{N}\right), \\
& \theta: F_{i} \rightarrow D_{i-1} ; x \mapsto \sum_{y \in \operatorname{Crit}_{i-1}(g)} n^{h_{N}^{\prime}, \alpha_{N}^{\prime}}(x,(y \times\{\epsilon / 2\})) y, \\
& \theta^{\prime}: D_{i} \rightarrow F_{i} ; x \mapsto \sum_{y \in \operatorname{Crit}_{i}\left(f_{N}\right)} n^{h_{N}^{\prime}, \alpha_{N}^{\prime}}((x \times\{1-\epsilon / 2\}), y) y, \\
& \psi: D_{i} \rightarrow z D_{i} ; x \mapsto \sum_{y \in \operatorname{Crit}_{i}(g)} n^{h_{N}^{\prime}, \alpha_{N}^{\prime}}((x \times\{1-\epsilon / 2\}),(y \times\{\epsilon / 2\})) y .
\end{aligned}
$$

By analogy with Proposition 1.27:

Proposition 2.8. Let $(f, \alpha): M \rightarrow S^{1}$ be a Morse-Smale function, with $0 \in S^{1}$ a regular value.

(i) For every Morse-Smale function $(g, \beta): N=f^{-1}(0) \rightarrow \mathbb{R}$ with $\beta=\left.\alpha\right|_{N}$ and every $\delta>0$ there exists an $(\epsilon, \tau)$-splitting of $(h, \alpha)$ along $(g, \beta)$ such that $\|h-f\|_{0} \leq \delta$.

(ii) The Novikov complex of a splitting $(h, \alpha)$ of $(f, \alpha)$ along $(g, \beta)$ with algebraic cobordism $\Gamma_{N}(M, h, \alpha)=\left(F, D, \theta, \theta^{\prime}, \psi\right)$ is given by

$$
C^{N o v}(M, h, \alpha)=E
$$

with $E$ the based f.g. free $\mathbb{Z}[\pi]_{\zeta}((z))$-module chain complex defined by

$$
\begin{aligned}
& d_{E}=\left(\begin{array}{ccc}
-d_{D} & 0 & 0 \\
1-z \psi & d_{D} & \theta \\
-z \theta^{\prime} & 0 & d_{F}
\end{array}\right): \\
& E_{i}=\left(D_{i-1} \oplus D_{i} \oplus F_{i}\right)_{\zeta}((z)) \rightarrow E_{i-1}=\left(D_{i-2} \oplus D_{i-1} \oplus F_{i-1}\right)_{\zeta}((z)) .
\end{aligned}
$$


Proof. (i) As for 2.8 (i).

(ii) The Morse complex of $(\bar{h}, \bar{\alpha}): \bar{M} \rightarrow \mathbb{R}$ is

$$
\begin{aligned}
d_{C(\bar{M}, \bar{h}, \bar{\alpha})}=\left(\begin{array}{ccc}
-d_{D} & 0 & 0 \\
1-z \psi & d_{D} & \theta \\
-z \theta^{\prime} & 0 & d_{F}
\end{array}\right): \\
C(\bar{M}, \bar{h}, \bar{\alpha})_{i}=\left(D_{i-1} \oplus D_{i} \oplus F_{i}\right)_{\zeta}\left[z, z^{-1}\right] \\
\rightarrow C(\bar{M}, \bar{h}, \bar{\alpha})_{i-1}=\left(D_{i-2} \oplus D_{i-1} \oplus F_{i-1}\right)_{\zeta}\left[z, z^{-1}\right]
\end{aligned}
$$

and

$$
C^{N o v}(M, h, \alpha)=E=\mathbb{Z}[\pi]_{\zeta}((z)) \otimes_{\mathbb{Z}[\pi]_{\zeta}\left[z, z^{-1}\right]} C(\bar{M}, \bar{h}, \bar{\alpha})
$$

In the setting of Proposition 2.8 define a based f.g. free $\mathbb{Z}[\pi]_{\zeta}((z))$-module chain complex $\widehat{F(h)}$ by

$$
\begin{aligned}
d_{\widehat{F(h)}}=d_{F}+z \theta^{\prime}(1-z \psi)^{-1} \theta & =d_{F}+\sum_{j=0}^{\infty} z^{j+1} \theta^{\prime} \psi^{j} \theta: \\
\widehat{F(h)} & \left.=\left(F_{i}\right)_{\zeta}((z)) \rightarrow \widehat{F(h}\right)_{i-1}=\left(F_{i-1}\right)_{\zeta}((z)) .
\end{aligned}
$$

The role of this complex is to eliminate in a purely algebraic way the contribution of $g$ from the Novikov complex of the splitting $(h, \alpha)$. Clearly, this suggests that $\widehat{F(h)}$ is a good approximation of $C^{N o v}(M, f, \alpha)$ and we shall see further that this is indeed the case.

It is convenient to fix the notations

$$
\mathcal{R}=\mathbb{Z}[\pi]_{\zeta}((z)), \quad \mathcal{R}^{\prime}=\mathbb{Z}[\pi]_{\zeta}[[z]] \subset \mathcal{R}
$$

All complexes and isomorphisms in this result are understood to belong to the category of $\mathcal{R}$-module chain complexes.

Definition 2.9. An isomorphism of based f.g. free $\mathcal{R}$-modules $\Theta: F \rightarrow G$ is $\mathcal{R}^{\prime}$-simple if

$$
\tau(\Theta) \in \operatorname{im}\left(K_{1}\left(\mathcal{R}^{\prime}\right) \rightarrow K_{1}(\mathcal{R})\right)
$$

The result below is the $S^{1}$-analogue of the Rigidity Theorem 1.19 (iii) and the gluing Theorem 1.29.

Theorem 2.10. Fix the Morse-Smale function $(f, \alpha): M \rightarrow S^{1}$.

(i)There exists $\delta_{f}>0$ such that if $\left(f^{\prime}, \alpha^{\prime}\right)$ is a Morse-Smale function with $\| f^{\prime}-$ $f \|_{0} \leq \delta_{f}$, then $C^{\text {Nov }}(M, f, \alpha)$ is a retract of $C^{N o v}\left(M, f^{\prime}, \alpha^{\prime}\right)$. In particular, the $\mathcal{R}^{\prime}$-simple isomorphism type of the Novikov complex of $(f, \alpha)$ is independent of the choice of $\alpha$. 
(ii) For any $(\epsilon, \tau)$-splitting $(h, \alpha): M \rightarrow S^{1}$ of $(f, \alpha)$ along a Morse-Smale function $(g, \beta): N \rightarrow \mathbb{R}$ with $\epsilon, \tau$ sufficiently small there is an $\mathcal{R}^{\prime}$-simple isomorphism

$$
\Theta: C^{N o v}(M, f, \alpha) \stackrel{\cong}{\rightarrow} \widehat{F(h)} \text {. }
$$

(iii) For any $n \in \mathbf{N}$ there exist Morse-Smale functions $\left(g_{n}, \beta\right): N \rightarrow \mathbb{R}$ and splittings $\left(h_{n}, \alpha\right): M \rightarrow S^{1}$ of $(f, \alpha)$ along $\left(g_{n}, \beta\right)$ such that there is an $\mathcal{R}^{\prime}$-simple isomorphism

$$
\Theta^{n}: C^{N o v}(M, f, \alpha) \stackrel{\cong}{\rightrightarrows F\left(h_{n}\right)}
$$

with $\Theta^{n}(x)-x \in z^{n} \mathcal{R}^{\prime}\left[\operatorname{Crit}_{*}(f)\right]$ for each basis element $x$.

Remark 2.11. The complex $\widehat{F(h)}$ behaves as if it were the Novikov complex $C^{N o v}(M, \widehat{f}, \alpha)$ of a Morse-Smale function $(\widehat{f}, \alpha): M \rightarrow S^{1}$ obtained from a splitting $(h, \alpha)$ by cancelling the pairs of critical points added to those of $f$ in the construction of $h$, with

$$
\mathrm{Crit}_{*}(\widehat{f})=\mathrm{Crit}_{*}(f) .
$$

Indeed, as in the real-valued case (see Remark 1.30) such a function does exist (this follows from Hutchings' extension in [10] of the bifurcation analysis of Laudenbach [13]) and one may construct it such that it is $C^{0}$-close to $f$. Using point (i) of the theorem and the existence of such an $\widehat{f}$ is sufficient to prove (ii) but, again as in the real-valued case, it is not enough for (iii). In the following we shall prove the whole statement independently of bifurcation considerations.

Proof. Order the critical points of $f_{N}$ following their critical values. Explicitly, we fix a total order on the finite set $S=\operatorname{Crit}_{*}\left(f_{N}\right)=\mathrm{Crit}_{*}(f)$ such that $x \leq y$ implies $f_{N}(x) \leq f_{N}(y)$ for $x, y \in S$.

The proof of the theorem uses the adaptation to the $S^{1}$-valued case of the techniques developed in the first three sections of this paper. These methods will produce certain morphisms relating the various chain complexes involved. Showing that these morphisms are isomorphisms is less immediate than in the $\mathbb{R}$-valued case, but it will always follow from the fact that our morphisms satisfy the assumptions of the rather obvious lemma below.

Lemma 2.12. Consider the free $\mathcal{R}$-module $\mathcal{R}\{S\}$ generated by a finite ordered set $S$. An $\mathcal{R}$-module endomorphism $\Theta: \mathcal{R}\{S\} \rightarrow \mathcal{R}\{S\}$ such that for each $x \in S$

$$
\Theta(x)-x \in z \mathcal{R}^{\prime}\{S\}+\mathcal{R}^{\prime}\{y \in S: y<x\}
$$

is an $\mathcal{R}^{\prime}$-simple automorphism.

Proof. Write $S=\left\{x_{1}, x_{2}, \ldots, x_{k}\right\}$, with $x_{i}<x_{j}$ for $i<j$. The matrix of $\Theta$ has entries in $\mathcal{R}^{\prime}$, of the form

$$
\Theta=\left(\begin{array}{ccccc}
1+a_{11} & b_{12} & b_{13} & \ldots & b_{1 k} \\
a_{21} & 1+a_{22} & b_{23} & \ldots & b_{2 k} \\
a_{31} & a_{32} & 1+a_{33} & \ldots & b_{3 k} \\
\vdots & \vdots & \vdots & \ddots & \vdots \\
a_{k 1} & a_{k 2} & a_{k 3} & \ldots & 1+a_{k k}
\end{array}\right)
$$


with $a_{i j} \in z \mathcal{R}^{\prime}=z \mathbb{Z}[\pi]_{\zeta}[[z]], b_{i j} \in \mathcal{R}^{\prime}$. The augmentation

$$
\epsilon: \mathcal{R}^{\prime} \rightarrow \mathbb{Z}[\pi] ; z \mapsto 0
$$

sends $\Theta$ to an upper triangular matrix of the form

$$
\epsilon(\Theta)=\left(\begin{array}{ccccc}
1 & \epsilon\left(b_{12}\right) & \epsilon\left(b_{13}\right) & \ldots & \epsilon\left(b_{1 k}\right) \\
0 & 1 & \epsilon\left(b_{23}\right) & \ldots & \epsilon\left(b_{2 k}\right) \\
0 & 0 & 1 & \ldots & \epsilon\left(b_{3 k}\right) \\
\vdots & \vdots & \vdots & \ddots & \vdots \\
0 & 0 & 0 & \ldots & 1
\end{array}\right)
$$

which is clearly invertible. The matrix defined by

$$
\Psi=\Theta \epsilon(\Theta)^{-1}-I
$$

has entries in $z \mathcal{R}^{\prime}$. Thus $\Theta$ itself is invertible, with inverse

$$
\Theta^{-1}=\epsilon(\Theta)^{-1}\left(1+\sum_{j=1}^{\infty}(-\Psi)^{j}\right)
$$

defined over $\mathcal{R}^{\prime}$.

We now return to the proof of Theorem 2.10. The first remark is that the Definitions $1.1,1.6$ and 1.10 apply ad literam to the case of $S^{1}$-valued maps because the relevant conditions are local in nature. Similarly, the statements in Proposition 1.9 (i) and Proposition 1.11 (iii) remain valid if we replace the relevant Morse complexes by the corresponding Morse-Novikov complexes with one exception: the argument given for the proof of 1.11 (iii) only shows that $f^{M}$ is a chain map in the $S^{1}$-valued case.

More care is necessary for the constructions of Morse cobordisms.

Lemma 2.13. (i) Let $\left(f_{0}, \alpha_{0}\right),\left(f_{1}, \alpha_{1}\right): M \rightarrow S^{1}$ be two Morse-Smale functions with $M$ closed. Suppose that $f_{0}$ and $f_{1}$ are homotopic. Then there exists $c \in \mathbb{R}$ and a simple Morse cobordism $\left(F, \alpha^{\prime}\right): M \times[0,1] \rightarrow S^{1}$ between $\left(e^{2 \pi i c} f_{0}, \alpha_{0}\right)$ and $\left(f_{1}, \alpha_{1}\right)$.

(ii) If there exists a constant $c \geq 0$ such that

$$
\left(f_{1}, \alpha_{1}\right)=\left(e^{2 \pi i c} f_{0}, \alpha_{0}\right): M \rightarrow S^{1}
$$

then there exists a cobordism $\left(F, \alpha^{\prime}\right): M \times[0,1] \rightarrow S^{1}$ between $f_{0}$ and $f_{1}$ such that $F^{M \times[0,1]}$ is the identity.

Proof. The difference between proving this statement and the constructions in Sect. 1.1.3 comes from the fact that the formulas for the homotopies given there are no longer applicable in this case. Let $\mu: S^{1} \times \mathbb{R} \rightarrow S^{1}$ be given by $\mu(\tau, t)=e^{2 \pi i t} \tau$. We also use the following convention: $d \tau \in \Omega^{1} S^{1}$ is the standard volume form on $S^{1}$ and for an $S^{1}$-valued function $g$ we let $d g=g^{*}(d \tau)$. 
(i) Let $F^{\prime}: M \times[0,1] \rightarrow S^{1}$ be the homotopy of $f_{0}$ and $f_{1}$. By a simple partition of unity argument we may assume that $F^{\prime}$ is flat at the ends of the cobordism in the sense that there are collared neighbourhoods $U_{i} \approx M \times\left[a_{i}, a_{i}+\delta\right]$ of $M \times\{i\}$, $i \in\{0,1\}, a_{0}=0, a_{1}=1-\delta, \delta$ small, such that for $(x, t) \in U_{i}$ we have $F^{\prime}(x, t)=$ $f_{i}(x)$. Because $M$ is compact there exists $m$ such that $\left|\left(\partial F^{\prime} / \partial t\right)(x, t)\right| \leq m$ for all $(x, t) \in M \times[0,1]\left(\right.$ where $\left.\partial F^{\prime} / \partial t=\left\langle d F^{\prime}, \partial / \partial t\right\rangle\right)$. Let $k:(-1 / 2,3 / 2) \rightarrow \mathbb{R}$ be a Morse function with exactly two critical points, a minimum at 0 and a maximum at 1 that has value 1 and such that for $t \in[\delta, 1-\delta]$ we have $k^{\prime}(t)<-m$. Now define $F: M \times[0,1] \rightarrow S^{1}$ by $F=\mu\left(F^{\prime} \times k\right)$. As $F^{\prime}$ is flat close to $\partial(M \times[0,1])$, to verify that $F$ is a Morse cobordism we only need to notice that $F$ has no critical points in $M \times[0,1] \backslash\left(U_{0} \cup U_{1}\right)$. This happens because in this set $\partial F / \partial t=\partial F^{\prime} / \partial t+k^{\prime}<0$ (which is computed by using polar coordinates $\tau \rightarrow e^{2 \pi i \tau}$ on $S^{1}$ ). It is obvious that there are metrics $\alpha^{\prime}$ that extend $\alpha_{i}$ and such that $\left(F, \alpha^{\prime}\right)$ is Morse-Smale.

(ii) This is the analogue of the construction of linear Morse cobordisms in Lemma 1.17. We consider a Morse function $k:(-1 / 2,3 / 2) \rightarrow \mathbb{R}$ as above (without any derivative restrictions). Let $v:[0,1] \rightarrow[0,1]$ be an increasing $C^{\infty}$-function with $v([0, \delta])=0, v([1-\delta, 1])=1$. We let $F^{\prime}: M \times[0,1] \rightarrow S^{1}$ be a smooth homotopy of $f_{0}$ and $f_{1}$ that is defined by $F^{\prime}(x, t)=e^{2 \pi i v(t)} f_{0}(x)$. Finally, we define $F$ as before $F(x, t)=\mu\left(F^{\prime} \times k\right)$. We take on $M \times[0,1]$ the metric $\alpha^{\prime}=\alpha_{0}+d t^{2}$. It is easy to verify that $(F, \beta)$ is a cobordism whose induced morphism is the identity. Indeed, the lift of this pair to $\bar{F}: \bar{M} \times[0,1] \rightarrow \mathbb{R}$ is an obvious linear cobordism in the real valued sense and this implies that $F^{M \times[0,1]}$ is the identity.

(i) Given these two lemmas item (i) of Theorem 2.10 follows exactly by the same argument as item (ii) of Theorem 1.19. More precisely, assume that $\left(f_{i}, \alpha_{i}\right): M \rightarrow S^{1}$ are homotopic Morse-Smale functions such that $f_{1}$ is $C^{0}$ close to $f_{0}$. Then there exists a homotopy $F^{\prime}: M \times[0,1] \rightarrow S^{1}$ of $f_{0}$ to $f_{1}$ which is $C^{0}$-close to the constant homotopy $f_{0}$. This means that by taking $f_{1}$ sufficiently $C^{0}$ close to $f_{0}$ we may also assume the constant $c$ in Lemma 2.13 (i) to be as small as desired. We now construct Morse cobordisms relating $e^{2 \pi i c^{\prime}} f_{0}, e^{2 \pi i c} f_{1}$ and $f_{0}$ for small constants $c$ and $c^{\prime}$ that are similar to those in Lemma 1.21 and the argument in Lemma 1.22 applied to the lifts of $\bar{f}_{0}, \bar{f}_{1}$ to $\bar{M}$ implies that the resulting chain maps

$$
\begin{aligned}
& w: C^{N o v}\left(M, f_{0}, \alpha_{0}\right) \rightarrow C^{N o v}\left(M, f_{1}, \alpha_{1}\right), \\
& w^{\prime}: C^{N o v}\left(M, f_{1}, \alpha_{1}\right) \rightarrow C^{N o v}\left(M, f_{0}, \alpha_{0}\right)
\end{aligned}
$$

have the property that $w^{\prime} \circ w$ satisfies the assumptions of Lemma 2.12 and is therefore an isomorphism. If $f_{0}=f_{1}$ the fact that $w^{\prime}$ is surjective implies that it is an isomorphism (because any module epimorphism between finitely generated free modules of the same rank is an isomorphism).

(ii) Again, the proof here consists of adapting the proof of Theorem 1.29 (i) to the $S^{1}$-valued case. We shall use the notations introduced in Definition 2.6. In particular $f$ and $h$ only differ in the interior of a small tubular neighbourhood $U=N \times[-\epsilon, \epsilon]$ and on this set, if we identify the interval $[-\epsilon, \epsilon]$ with a small arc we have $f(x, t)=t$. By taking $\tau$ small we can make the function $\left.h\right|_{U}$ as $C^{0}$ close to the function $\left.f\right|_{U}$ as desired. Therefore, we may find a small constant $l$ such that 
$h(x, t)<t+l=f(x, t)+l$. We then construct by the methods in Lemma 1.17 a Morse function $F^{1}: U \times[0,1] \rightarrow[-\epsilon, \epsilon+c]$ which is of the form $(1-u(t)) h+$ $u(t)(f+l)$ with $u(1)=1, u(0)=0, u^{\prime}(0)=u^{\prime}(1)=0, u^{\prime \prime}(0)>0>u^{\prime \prime}(1)$ and such that $\left(\partial F^{1} / \partial t\right)(y, t)<0$ if $(y, t) \in U \times(0,1)$. Because, $h$ and $f$ coincide outside $U$ we notice that on $\partial U$ we have $F^{1}(y, t)=f(y)+l u(t)$. Recalling that actually the image of $F^{1}$ is in $S^{1}$ we rewrite $\left.F^{1}\right|_{\partial U \times[0,1]}$ as $F^{1}(y, t)=e^{2 \pi i l u(t)} f$. This shows that $F^{1}$ extends to a function $H: M \times[0,1] \rightarrow S^{1}$ which is equal to $e^{2 \pi i l u(t)} f(y)$ if $(y, t) \in(M \backslash U) \times[0,1]$. With an appropriate metric $\alpha^{\prime}$ the function $H$ is a Morse cobordism between $(h, \alpha)$ and $\left(e^{2 \pi i l} f, \alpha\right)$. By inspecting its lift $\bar{H}$ we see that the induced morphism $T=H^{M \times[0,1]}: C^{N o v}(M, f, \alpha) \rightarrow C^{N o v}(M, h, \alpha)$ has the following property: for each generator $x \in \mathrm{Crit}_{*}(f)$ we have $T(x)=x+o(x)+z p$ with $o(x) \in \mathbb{Z}[\pi]\left\{\mathrm{Crit}_{*}(h) \backslash \mathrm{Crit}_{*}(f)\right\}$ and $p \in \mathbb{Z}[\pi]_{\zeta}[[z]]\left\{\mathrm{Crit}_{*}(h)\right\}$. We consider the composition $J^{\prime}=J_{h} \circ T$. The description of $J_{h}$ now shows that $J^{\prime}(x)=x+z q$ with $q \in \mathbb{Z}[\pi]_{\zeta}[[z]]\left\{\operatorname{Crit}_{*}(f)\right\}$. Lemma 2.12 implies that $J^{\prime}$ is an isomorphism.

(iii) We intend to prove this item by showing that for a fixed $n$ and some special choice of function $g_{n}$ leads by the construction above to a splitting $h_{n}$, a cobordism $\left(H_{n}, \alpha_{n}\right)$ and a corresponding morphism $T_{n}$ that has the form $T_{n}(x)=x+o_{n}(x)+$ $z^{n} p$ such that with the notations in Definition 2.6 we have

$$
p \in \mathbb{Z}[\pi]_{\zeta}[[z]]\left\{\operatorname{Crit}_{*}(h)\right\}
$$

and

$$
o_{n} \in \mathbb{Z}[\pi]_{\zeta}[[z]]\left\{\mathrm{Crit}_{*-1}\left(g_{n}\right) \times\{-\epsilon / 2\}\right\} .
$$

Notice that if such a $T_{n}$ is constructed, then as $J_{h_{n}}: C^{\text {Nov }}\left(M, h_{n}\right) \rightarrow \widehat{F\left(h_{n}\right)}$ vanishes on $\mathbb{Z}[\pi]_{\zeta}[[z]]\left\{\right.$ Crit $\left._{*-1}\left(g_{n}\right) \times\{-\epsilon / 2\}\right\}$ the composition $J_{h_{n}} \circ T_{n}$ is an isomorphism of the form required. Therefore, we have reduced the proof to the construction of $h_{n}, H_{n}$ such that $T_{n}$ is as above. This construction may be accomplished in a way similar to the proof of Theorem 1.29 (iii). We recall that the metric $\alpha$ is the product metric $\beta+d t^{2}$ inside a neighbourhood $N \times[-\delta, \delta]$.

As in Theorem 1.29 (iii), the key point is to work with an adapted function $g$ (see Definition 1.28). In our setting, consider a Morse-Smale function $\left(g_{n}, \beta\right): N \rightarrow \mathbb{R}$ which is adapted to splitting the Morse-Smale function $\left(f_{N}(i), \alpha_{N}(i)\right): M_{N}(i) \rightarrow \mathbb{R}$ along $z^{i-1} N$ for all $0<i \leq n$ (the notations are as in Lemma 2.5). Such a function exists by the same argument as in the real valued case (there are more conditions to be verified but they are of the same type and finite in number). We now construct the $(\epsilon, \tau)$-splitting $h_{n}$ such that inside the set $N \times[-\epsilon, \epsilon]$ the function $h_{n}$ has the particular form of the functions $f_{\epsilon}$ in the proof of Proposition 1.27. There is one minor additional point that needs to be looked at: we need to be sure that $\left(h_{n}, \alpha\right)$ satisfies the Morse-Smale transversality condition. The needed transversality is certainly satisfied with respect to the unstable and stable manifolds of the critical points in $\mathrm{Crit}_{*}(h) \cup \mathrm{Crit}_{*}(f)$ by the transversality part in the "adapted" condition. The rest of the transversalities needed can be insured by possibly slightly perturbing the stable manifolds of $g_{n}$. This can preserve the transversalities already achieved and does not affect the part (i) of the adapted condition (see Definition 1.28).

We now construct the Morse cobordism $\left(H_{n}, \alpha_{n}\right): M \times[0,1] \rightarrow S^{1}$ in the same general way as for item (ii), but now we need to control the behaviour of 
the flow lines of $\bar{H}_{n}$ over the set $K=M_{N}(n) \backslash\left(z^{n}+z^{-1}\right)(N \times(-\epsilon,+\epsilon))$. More precisely, we shall construct a cobordism $\left(H_{n}, \alpha_{n}\right)$ which satisfies

$$
n^{\bar{H}_{n}}(x, y)=0
$$

for all $x \in \mathrm{Crit}_{k}(f) \times\{1\} \subset \bar{M} \times\{1\}$ and

$$
y \in z^{i}\left(\operatorname{Crit}_{k}(f) \cup\left(\operatorname{Crit}_{k}\left(g_{n}\right) \times\{\epsilon / 2\}\right)\right)
$$

for all $0<i \leq n-1$. This immediately implies our claim for $T_{n}$ (as in the proof of Theorem 1.29). The argument providing this $\left(H_{n}, \alpha_{n}\right)$ follows. It is quite similar to that in the proof of Theorem 1.29 (iii), but with the additional difficulty that all the constructions initially accomplished for real valued functions (on $\bar{M}$ ) will need to be adjusted such that they are periodic in order to descend to $M$.

We first make the properties of $h_{n}$ more explicit. We assume that the neighbourhood $N \times[-2 \epsilon, 2 \epsilon]$ is like in the definition of splittings (after reparametrization $f$ has the form $(x, t) \mapsto t$ and the metric is the product one here). On $N \times[-2 \epsilon, 2 \epsilon]$ we consider a Morse-Smale function $g^{\prime}$ such that $g^{\prime}(x, t)=t$ for $(x, t) \in N \times([-2 \epsilon,-\epsilon] \cup[\epsilon, 2 \epsilon]) ; g^{\prime}$ is a linear cobordism between $\epsilon\left(g_{n}+k\right)$ and $\epsilon\left(g_{n}+k^{\prime}\right)$ on $N \times[-\epsilon / 2, \epsilon / 2]$ and is a linear combination of the form $u(t)(-\epsilon)+(1-u(t)) \epsilon\left(g_{n}+k^{\prime}\right)$ inside $N \times[-\epsilon,-\epsilon / 2]$ with $u:[-\epsilon,-\epsilon / 2] \rightarrow \mathbb{R}$ an appropriate function with $u([-2 \epsilon-\epsilon)=1$ and $u(-\epsilon / 2)=0$ as in Lemma 1.13; a similar linear combination is valid inside $N \times[\epsilon / 2, \epsilon]$. The function $h_{n}$ equals $g^{\prime}$ on $N \times[-\epsilon, \epsilon]$ and is equal to $f$ on $M \backslash N \times[-\epsilon, \epsilon]$.

Similarly, we may define a standard cobordism $\left(H^{\prime}, \alpha^{\prime}\right)$ between $g^{\prime}$ and the function $(x, t) \mapsto t+c$ which is defined on $(N \times[-2 \epsilon, 2 \epsilon]) \times[0,1]$ and where $c$ is a very small constant depending on $k, k^{\prime}, \epsilon$. This cobordism $H^{\prime}$ differs from a linear cobordism only in the interior of $N \times[-\epsilon, \epsilon] \times[0, \epsilon]$. The metric $\alpha^{\prime}$ is the product metric $\alpha+d \tau^{2}$. We fix $\delta>0$ such that if $\left(x, t, t^{\prime}\right) \in N \times[-2 \epsilon, 2 \epsilon] \times[1-\delta, 1]$, then the flow line of $-\nabla^{\alpha^{\prime}} H^{\prime}$ containing $\left(x, t, t^{\prime}\right)$ does not intersect $N \times[-2 \epsilon, 2 \epsilon] \times$ $[0,2 \epsilon]$.

Now let $0<\epsilon_{i}<\epsilon<1 / 3$ and consider $\phi_{i}: N \times[-2 \epsilon, 2 \epsilon] \times[0,2 \epsilon] \rightarrow$ $N \times[-2 \epsilon, 2 \epsilon] \times[0,2 \epsilon]$ a diffeomorphism given by $\left(x,\left(t, t^{\prime}\right)\right) \mapsto\left(x, l_{i}\left(t, t^{\prime}\right)\right)$ where $l_{i}:[-2 \epsilon, 2 \epsilon] \times[-2 \epsilon, 2 \epsilon] \rightarrow[-2 \epsilon, 2 \epsilon] \times[-2 \epsilon, 2 \epsilon]$ is a diffeomorphism such that $l_{i}$ restricts to the identity on the complement of the square of side $[-(3 / 2) \epsilon,(3 / 2) \epsilon]$ and the image of the square of side $\left[-\epsilon_{i}, \epsilon_{i}\right]$ is the square of side $[-\epsilon, \epsilon]$. We also require that $l_{i}$ restricts to diffeomorphisms when $t=0$ and when $t^{\prime}=0$. Let $\left(H_{i}, h_{i}\right)=\left(\epsilon_{i} / \epsilon\right)\left(H^{\prime}, h^{\prime}\right) \circ \phi_{i}$. The metric associated to the Morse cobordism $H_{i}$ is the metric $\alpha^{i}=\phi_{i}^{*} \alpha^{\prime}$. It is useful to note that $H_{i}$ coincides with a linear cobordism outside of $N \times\left[-\epsilon_{i}, \epsilon_{i}\right] \times\left[0, \epsilon_{i}\right]$ and that $\nabla^{\alpha^{i}} H_{i}\left(x, t, t^{\prime}\right)$ and $\nabla^{\alpha^{\prime}} H_{i}\left(x, t, t^{\prime}\right)$ have the same projection on $N$. Clearly, $\alpha^{i}$ and $\alpha^{\prime}$ differ only outside $N \times[-(3 / 2) \epsilon,(3 / 2) \epsilon] \times[0,(3 / 2) \epsilon]$. For $0 \leq i \leq n$ we fix the following notation $K_{i}=z^{i}(N \times[-(3 / 2) \epsilon,(3 / 2) \epsilon])$ and $K^{\prime}=\bar{K} \backslash \cup K_{i}$.

We intend to construct a cobordism $\left(H^{\prime \prime}, \alpha^{\prime \prime}\right): K \times[0,1] \rightarrow \mathbb{R}$ between a Morse-Smale function $h^{\prime \prime}$ and $f_{N}(n)$ such that there exists a sequence of $\epsilon_{i}$ 's with the property that $H^{\prime \prime}$ is a linear cobordism on $K^{\prime} \times[0,1] \times[0,1]$, on $\left.\left(H^{\prime \prime}, \alpha^{\prime \prime}\right)\right|_{z^{i}(N \times[-\epsilon, \epsilon] \times[0,1])}$ it agrees with $\left(z^{i} H_{i}, \alpha^{i}\right)$ and it coincides with $z^{i} H^{\prime}$ 
on each set $z^{i}(N \times([-2 \epsilon,-(3 / 2) \epsilon] \cup[(3 / 2) \epsilon, 2 \epsilon]) \times[0,1])$. Moreover, $\left(H^{\prime \prime}, \alpha^{\prime \prime}\right)$ should verify the obvious analogue of (8).

To construct $H^{\prime \prime}$ we proceed by induction. We start with the trivial linear cobordism with a product metric. Assume now that we have already constructed $\left(\epsilon_{n-1}, \ldots \epsilon_{i}\right)$ and the function $h_{i}^{\prime}$ and $\left(H_{i}^{\prime}, \alpha_{i}^{\prime}\right)$ such that $\left.H_{i}^{\prime}\right|_{K_{j} \times[0,1]}=z^{j} H_{j}$ for all $j<i$ and $H_{i}^{\prime}$ is a cobordism between $\bar{f}$ and $h_{i}^{\prime}$ which is linear on $\left(K \backslash \cup_{j<i} K_{j}\right) \times$ $[0,1]$ and is such that the equation (8) is satisfied by $\left(H_{i}^{\prime}, \alpha_{i}^{\prime}\right)$. We now want to construct $\epsilon_{i-1}, H_{i-1}^{\prime}, \alpha_{i-1}^{\prime}$.

We first pick some choice for $R_{\epsilon^{\prime}}=H_{i-1}^{\prime}$ defined with respect to some $\epsilon_{i-1}=\epsilon^{\prime}$ and which satisfies the properties required from $H_{i-1}^{\prime}$ except possibly (8) and such that $\left.R_{\epsilon^{\prime}}\right|_{K_{i-1} \times[0,1]}=z^{i-1} H_{i-1}$ and $R_{\epsilon^{\prime}}=H_{i}^{\prime}$ in the complement of $K_{i-1} \times$ $[0,1]$. As in the Morse case we might need to modify the associated metric on $K \times[1 / 3,1-\delta]$ (in the real case it was enough to take $\delta=3 / 2$ but here it is useful to use the constant fixed above) such that $R_{\epsilon^{\prime}}$ is Morse-Smale (see above Lemma 1.31). In fact, it is easy to see that we may assume that the metric $\alpha_{\epsilon^{\prime}}$ associated to $R_{\epsilon^{\prime}}$ satisfies property (8) with respect to $H_{i}^{\prime}$ (because it can be chosen to be arbitrarily close to $\alpha_{i}^{\prime}$ ) in the exterior of $\left.K_{i-1} \times[0,2 \epsilon]\right)$ ), it coincides with the standard product metric outside $K \times[1 / 3,1-\delta] \cup \cup_{i} K_{i}$, it coincides with $\phi_{i}^{*}\left(\alpha^{\prime}\right)$ on $z^{j} N \times[-2 \epsilon, 2 \epsilon] \times[0,1], j \leq i-1$, and is arbitrarily close to the product metric $\alpha^{\prime}$. In fact, the set of choices for the metrics $\alpha_{\epsilon^{\prime}}$ is dense and open in some neighborhood of $\alpha^{\prime}$ viewed as a metric on $K^{\prime} \times[0,1]$. As the difference between $R_{\epsilon^{\prime}}$ and $H_{i}^{\prime}$ appears around $z^{i-1} N$, as in the proof of Lemma 1.31, we assume that there exists a pair of critical points $x, y$ of $R_{\epsilon^{\prime}}$ that do not satisfy (8) even if we make $\epsilon^{\prime} \rightarrow 0$. By using the induction hypothesis and proceeding as in the proof of that lemma (see also Remark $1.5 \mathrm{~b}$.) we are lead to the existence of a sequence of points $x_{1}, x_{2}, \ldots, x_{t}$ with the following properties: $x_{1}$ is a critical point of $f_{N}(n)$ such that $x=x_{1} \times\{1\}$,

$$
x_{2}, x_{3} \in \bigcup_{\operatorname{ind}_{g_{n}}(q)<\operatorname{ind}_{f}\left(x_{1}\right)} W_{g_{n}}^{u}(q) \subset z^{i-1} N,
$$

$x_{t}=y, x_{3}$ is related to $x_{4}$ by a flow line of $h_{i}^{\prime}, x_{1}$ is related to $x_{2}$ by a flow line of $\bar{f}, x_{2}$ is related to $x_{3}$ by a flow line of $g_{n}$ and all the points $x_{4}, \ldots, x_{t}$ are critical points of $h_{i}^{\prime}$ and appear as vertices in a sequence of broken flow lines of $h_{i}^{\prime}$ (in the proof of Lemma 1.31 this sequence only had 4 elements). Moreover, and this is a key point, all the points $x_{l}$ for $l>3$ belong to $W_{H_{i}^{\prime}}^{u}(x)$. By transversality this means that ind $h_{h_{i}^{\prime}}\left(x_{l}\right) \leq \operatorname{ind}\left(x_{1}\right)$. By the transversality properties of $g_{n}$ we have that

$$
\operatorname{ind}_{h_{i}^{\prime}}\left(x_{4}\right)<\operatorname{ind}_{f}\left(x_{1}\right)=\operatorname{ind}_{H_{i}^{\prime}}(x)-1=k-1 .
$$

Notice that this implies that ind $h_{h_{i}^{\prime}}\left(x_{t}\right)<\operatorname{ind}\left(x_{1}\right)$ which contradicts our assumption that ind $(y)=k-1$. Indeed, if $t \stackrel{i}{=} 4$ this has already been shown above and if $t>4$ then $y$ is in the unstable manifold of the point $x_{t-1}$ whose index is at most $k-1$. Again by transversality the index of $y$ is at most $k-2$. Therefore, the existence of the $\epsilon_{i}$ has been established and hence the construction of $\left(H^{\prime \prime}, \alpha^{\prime \prime}\right), h^{\prime \prime}$ follows by recurrence. 
In order to complete the proof we need to adjust $H^{\prime \prime}, h^{\prime \prime}, \alpha^{\prime \prime}$ in such a way that the validity of equation (8) is preserved, but the new functions and metric are periodic and hence induce corresponding notions on $M$. Let $\psi: K \times[0,1] \rightarrow$ $K \times[0,1]$ be a diffeomorphism which leaves invariant $\left(K-\cup z^{i}(N \times[-2 \epsilon, 2 \epsilon])\right) \times$ $[0,2 \epsilon]$ and is the inverse of $\phi_{i}$ on each set $\left.z^{i}(N \times[-2 \epsilon, 2 \epsilon]) \times[0,2 \epsilon]\right)$. Consider the composition $Q=H^{\prime \prime} \circ \psi$. This function still satisfies (8) but, of course, with respect to the metric $\alpha^{*}=\psi^{*}\left(\alpha^{\prime \prime}\right)$. Notice that $\alpha^{*}=\alpha^{\prime}$ in $\cup_{i}\left(K_{i} \times[0,1]\right) \cup$ $(K \times[1-\delta, 1] \cup[0,1 / 3])$ and so it is immediate to see that we can choose $\alpha^{\prime \prime}$ as described above on $K^{\prime} \times[0,1]$ but also such that $\alpha^{*}$ is periodic. On each of the sets $z^{i}(N \times[-2 \epsilon, 2 \epsilon] \times[0,1])$ the function $Q$ satisfies $Q\left(x, t, t^{\prime}\right)=u_{i}(t) z^{i} H^{\prime}\left(x, t, t^{\prime}\right)$ where $u_{i}(t)$ equals 1 on $[-2 \epsilon,-(3 / 2) \epsilon] \cup[(3 / 2) \epsilon, 2 \epsilon]$ and equals $\epsilon_{i} / \epsilon$ on $[-\epsilon, \epsilon]$ (these functions $u_{i}$ appear when the cobordisms $H_{i}$ are pasted with the linear cobordisms on $\left.K^{\prime} \times[0,1]\right)$. This means that there exists a diffeomorphism $v$ : $[-n, 1] \rightarrow[-n, 1]$ which satisfies $v(t)=t$ for $t \notin \cup[k-2 \epsilon, k+2 \epsilon]$ and with the property that for $\left(x, t, t^{\prime}\right) \in(N \times[k-2 \epsilon, k+2 \epsilon] \times[0,1])$, we have $v \circ Q\left(x, t, t^{\prime}\right)=$ $H^{\prime}\left(x, t, t^{\prime}\right)+k$ (we have identified everywhere $z^{i} f(x)$ and $\left.f(x)+i\right)$. We now define $H^{\prime \prime \prime}=v \circ Q$. By factorization this cobordism provides the desired cobordism $H_{n}: M \times[0,1] \rightarrow S^{1}$. Indeed, by construction, $\bar{H}_{n}$ satisfies the required properties relative to equation (8) with respect to the metric $\alpha^{*}$. The pair $\left(H_{n}, \alpha^{*}\right)$ might not be a Morse-Smale pair even if the Morse-Smale condition is satisfied up to order $n$. Therefore, we obtain the metric $\alpha_{n}$ by a new (and last) perturbation of $\alpha^{*}$ away from $M \times\{0,1\}$ such that $\alpha_{n}$ is sufficiently close to $\alpha^{*}$ for condition (8) to continue to be satisfied (this is possible because this condition concerns only the behavior of flow lines of "length" $n$ ). On $M \times\{0,1\}$ the metric $\alpha_{n}$ coincides with $\alpha$. Moreover, $\left.H_{n}\right|_{M \times\{1\}}$ is equal to $f$ up to a constant and $\left.H_{n}\right|_{M \times\{0\}}$ is a splitting of $f$. By construction the morphism induced by $H_{n}$ satisfies equation (8), and this concludes the proof.

Remark 2.14. (a) For any fixed Morse-Smale function $(f, \alpha): M \rightarrow S^{1}$, a splitting $(h, \alpha)$ of $(f, \alpha)$ along $g: N=f^{-1}(0) \rightarrow \mathbb{R}$ can be viewed as a geometric approximation of finite nature for $(f, \alpha)$. Indeed, $(f, \alpha)$ and $(h, \alpha)$ are compared by a simple Morse cobordism and the flow lines of $h$ are never longer than one fundamental domain. Theorem 2.10 (ii) indicates that the isomorphism type of the Novikov complex of $(f, \alpha)$ can be recovered from any such finite approximation $h$ that is sufficiently $C^{0}$-close to $f$. It is important to note that $g$ can be here any MorseSmale function $g: N \rightarrow \mathbb{R}$. Theorem 2.10 (iii) shows that by using $\ell$-adapted $g$ 's in the sense of being adapted to splitting $f_{N}(\ell)$ along $z^{\ell-1} N$ for higher and higher $\ell$ 's we reconstruct the Novikov complex itself. Producing $\ell$-adapted $g$ 's is a finite process that only depends on $f_{N}(\ell)$ and its difficulty increases with $\ell$. As in any approximation process more effort is needed to produce better approximations.

(b) A natural question is whether there is some $g: N \rightarrow \mathbb{R}$ and an associated splitting $h(g)$ such that there exists an isomorphism

$$
\left.\Theta^{\infty}: C^{N o v}(M, f, \alpha) \rightarrow \widehat{F(h(g)}\right)
$$

which is basis preserving. The major difficulty in proving such a statement along the method above comes from the fact that one would need $g$ to be adapted to 
splitting $f_{N}(\ell): M_{N}(\ell) \rightarrow \mathbb{R}$ along $z^{\ell-1} N$ for any $\ell$. The existence of such a $g$ is not at all clear. It is also possible to use a slightly less restrictive condition at the point (ii) in Definition 1.28 but as it turns out this leads to some technical difficulties related to transversality issues (in the $S^{1}$-valued case).

(c) The work of Pajitnov [16] shows that for a certain class $\mathcal{L}$ of pairs $(f, \alpha)$ which is $C^{0}$-dense and open in the set of Morse-Smale pairs, there exists an algebraic cobordism $\left(F, D, \theta, \theta^{\prime}, \psi\right)$ such that the differentials of the Novikov complex of $(f, \alpha)$ are described by the formula

$$
\begin{aligned}
& d_{C^{N o v}(M, f, \alpha)}=d_{F}+\sum_{j=0}^{\infty} z^{j+1} \theta^{\prime} \psi^{j} \theta: \\
& C^{N o v}(M, f, \alpha)_{i}=\left(F_{i}\right)_{\zeta}((z)) \rightarrow C^{N o v}(M, f, \alpha)_{i-1}=\left(F_{i-1}\right)_{\zeta}((z)) .
\end{aligned}
$$

with $\psi: D_{i} \rightarrow z D_{i}$ constructed by means of a homological version of the partially defined flow-return. Although $\left(F, D, \theta, \theta^{\prime}\right)$ was not constructed from a splitting it certainly suggests that, at least for this class of functions, the answer to the question discussed in (b) is positive. This seems even more likely because the property that distinguishes $(f, \alpha) \in \mathcal{L}$ from the rest of Morse-Smale functions appears to be quite close to demanding the existence of an "infinitely" adapted $g$. It should be noted that it is a rather difficult task to decide whether a particular Morse-Smale function $(f, \alpha): M \rightarrow S^{1}$ belongs to $\mathcal{L}$ or not. Therefore, Theorem 2.10 is a result essentially complementary to the results in [16] because 2.10 applies to all MorseSmale functions $(f, \alpha)$ the only choices involved having to do with the auxiliary function $g$.

(d) As in Farber and Ranicki [3] define the Cohn localization $\Sigma^{-1} \mathbb{Z}[\pi]_{\zeta}\left[z, z^{-1}\right]$ of $\mathbb{Z}[\pi]_{\zeta}\left[z, z^{-1}\right]$ inverting the set $\Sigma$ of square matrices over $\mathbb{Z}[\pi]_{\zeta}[z]$ sent to invertible matrices over $\mathbb{Z}[\pi]$ by the augmentation $z \mapsto 0$. Let $\left(F, D, \theta, \theta^{\prime}, \psi\right)$ be the algebraic cobordism over $\mathbb{Z}[\pi]$ determined by a splitting $(h, \alpha)$ of $(f, \alpha)$ as in Theorem 2.10, with

$$
D=C(N, g, \beta), F=C\left(M_{N}, f_{N}, \alpha_{N}\right) .
$$

The rational Novikov complex $C^{F R}(M, f, \alpha)$ of [3] is the based f.g. free $\Sigma^{-1} \mathbb{Z}[\pi]_{\zeta}\left[z, z^{-1}\right]$-module chain complex defined algebraically by

$$
C^{F R}(M, f, \alpha)=\left(\Sigma^{-1}\left(F_{i}\right)_{\zeta}\left[z, z^{-1}\right], d_{F}+z \theta^{\prime}(1-z \psi)^{-1} \theta\right)
$$

The rational analogue of Theorem 2.10 shows that the basis-preserving isomorphism type of $C^{F R}(M, f, \alpha)$ is independent of the choice of generic metric $\alpha$, and gives an isomorphism

$$
\mathbb{Z}[\pi]_{\zeta}((z)) \otimes_{\Sigma^{-1} \mathbb{Z}[\pi]_{\zeta}\left[z, z^{-1}\right]} C^{F R}(M, f, \alpha) \cong C^{N o v}(M, f, \alpha)
$$

See [17] for further details of this isomorphism, and of the construction of $C^{F R}(M, f, \alpha)$. 


\section{References}

1. Abbondandolo, A., Majer, P.: Morse homology on Hilbert spaces. Commun. Pure Appl. Math. 54, 689-760 (2001)

2. Cieliebak, K., Floer, A., Hofer, H.: Symplectic homology II: A general construction. Math. Z. 218, 103-122 (1995)

3. Farber, M., Ranicki, A.A.: The Morse-Novikov theory of circle-valued functions and noncommutative localization. E-print http://arXiv.org/abs/math.DG/9812122, Proc. 1998 Moscow Conference for S.P. Novikov's 60th Birthday. Proc. Steklov Inst. 225, 381-388 (1999)

4. Floer, A., Hofer, H.: Coherent orientations for periodic orbit problems in symplectic geometry. Math. Z. 212, 13-38 (1993)

5. Floer, A., Hofer, H.: Symplectic homology I: Open sets in $\mathbb{C}^{n}$. Math. Z. 215, 37-88 (1994)

6. Floer, A., Hofer, H., Salamon, D.: Transversality in elliptic Morse theory for the symplectic action. Duke Math. J. 80, 251-292 (1996)

7. Franks, J.: Morse-Smale flows and homotopy theory. Topology 18, 199-215 (1979)

8. Hirsch, M.W.: Differential Topology. Grad. Texts Math. 33. Springer 1976

9. Hofer, H., Zehnder, E.: Symplectic Invariants and Hamiltonian Dynamics. Birkhäuser 1994

10. Hutchings, M.: Reidemeister torsion in generalized Morse theory. Forum Math. 14, 209-244 (2002). E-print http://arXiv.org/abs/math.DG/9907066

11. Hutchings, M., Lee, Y.-J.: Circle-valued Morse theory and Reidemeister torsion. Geom. Topol. 3, 369-396 (1999)

12. Latour, F.: Existence de 1-formes fermées non singulières dans une classe de cohomologie de de Rham. Publ. Math., Inst. Hautes Études Sci. 80, 135-194 (1995)

13. Laudenbach, F.: On the Thom-Smale complex. Astérisque 205, 219-233 (1992)

14. Milnor, J.: Morse Theory. Ann. Math. Stud. 51. Princeton 1963

15. Novikov, S.P.: The hamiltonian formalism and a multi-valued analogue of Morse theory. Uspeki Mat. 37, 3-49 (1982). English tr. Russ. Math. Surv. 37, 1-56 (1982)

16. Pajitnov, A.: $C^{0}$-generic properties of the boundary operators in the Novikov complex. E-print math.DG/9812157. A.M.S. Translations 197, 29-117 (1999)

17. Ranicki, A.: The algebraic construction of the Novikov complex of a circle-valued Morse function. Math. Ann. 322, 745-785 (2002).

E-print http://arXiv.org/abs/math.dg-ga/9903090

18. Salamon, D.: Lectures on Floer Homology. Symplectic Geometry and Topology, ed. by Y. Eliashberg, L. Traynor, IAS/Park City Mathematics series 7, 143-230 (1999)

19. Salamon, D., Zehnder, E.: Morse theory for periodic solutions of Hamiltonian Systems and the Maslov index. Commun. Pure Appl. Math. 45, 1303-1360 (1992) 